\title{
TOPOLOGICAL DYNAMICS
}

\author{
BY WILLIAM A. VEECH ${ }^{1}$
}

\section{Table of Contents}

\section{Dynamical embedding}

1.1. Uniformly distributed sequence generators

1.2. Weak mixing relative to a partition

1.3. Construction of $P$-mixing transformations

1.4. Well distributed sequences

1.5. A theorem in Diophantine approximation

1.6. Disjointness in topological dynamics

1.7. Proof of Theorem 1.5.1

1.8. Quasiregular points and generic points

1.9. Normal sequences and collectives

1.10. Disjointness in ergodic theory

1.11. The theorems of Weiss, Kamae, and Rauzy

1.12. Regularity and irregularity of uniform distribution

1.13. The construction of prime flows

1.14. Interval exchange transformations

2. The structure of minimal flows

2.1. Statements of results

2.2. Generalities on flows

2.3. The functor $2^{\text {:x }}$

2.4. Almost periodic extensions

2.5. Topologies associated to minimal flows

2.6. The relativized equicontinuous structure relation

2.7. Another proximity relation

2.8. The canonical tower

\section{Appendix}

\section{Bibliography}

Introduction. This will be a survey of recent developments in two areas of topological dynamics, "dynamical embedding" (Part 1) and the structure of minimal flows (Part 2). Part 2 contains some results published here for the first time.

\section{Dynamical embedding}

Any sequence with values in a topological space or a probability space may be viewed as a "sample sequence" of a (nonunique) dynamical system or

An expanded version of an address delivered before the Houston meeting of the Society on November 23, 1974, at the invitation of the Committee to select hour speakers for Western regional meetings; received by the editors August 5, 1976.

AMS (MOS) subject classifications (1970). Primary 10K05, 10K25, 10K30, 10K35, 28A65, 28A70, 54H20.

' Research supported by NSF-MCS76-07094. 
stationary stochastic process. Such a general observation is of little value, but there are a number of instances in which the structure of the associated system is such as to yield new information about the original sequence. I shall describe a number of applications of this principle, most of which have sprung from ideas of Furstenberg, in \$§1.1-1.14. But first I shall make brief mention of a recent paper by Furstenberg (received after the present paper was typed), which provides a quick illustration.

A set $A$ of integers has positive upper density if there exist $\alpha>0$ and arbitrarily long intervals $I$ such that $I$ contains at least $\alpha|I|$ elements of $A$, $|I|=$ length of $I$. If $A$ has bounded gaps (i.e. is "relatively dense"), one may take $\alpha=L^{-1}$, where $L$ is the maximum gap. Van der Waerden's theorem, one of Khinchin's "three pearls of number theory" [163], implies that every relatively dense set of integers contains arbitrarily long (finite) arithmetic progressions. It was conjectured by Erdös and Turán that the same conclusion obtains for sets with positive upper density. Roth [162] proved a set with positive upper density contains arithmetic progressions of length three (a nontrivial result!), and Szemerédi, after settling the case of progressions of length four, proved the general result

TheOREM A (SZemerédi [160]). Let $A$ be a set of positive integers having positive upper density. Then A contains arithmetic progressions of arbitrary (finite) length.

In [161] Furstenberg makes an equivalent formulation of the Erdös-Turán conjecture, in the language of ergodic theory, and proves the conjecture in this formulation. To describe the basic idea, let $X=\{0,1\}^{\mathbf{Z}}$, and let $T$ be the left shift on $X(T x(n)=x(n+1), x(\cdot) \in X)$. Identify $A \subseteq \mathbf{Z}$ with the point $x_{A} \in X, x_{A}(n)=\chi_{A}(n), n \in \mathbf{Z}$, where $\chi$ denotes characteristic function. Then let $X_{A}$ be closure $\left\{T^{n} x_{A} \mid n \in \mathbf{Z}\right\}$. It is straightforward to check that if $y \in X_{A}$, then any block $\{y(n) \mid-M \leqslant n \leqslant M\}$ occurs somewhere in $x_{A}$ (in fact, $X_{A}$ is the set of points with this property). Thus, to say $A$ contains an arithmetic progression of length $k$ is to say there exist $y \in X_{A}$ and $n>0$ such that $y(0)=y(n)=y(2 n)=\cdots=y((k-1) n)=1$; what is the same, if $U_{A}=\left\{y \in X_{A} \mid y(0)=1\right\}$, then $U_{A} \cap T^{n} U_{A} \cap \cdots \cap T^{(k-1) n} U_{A} \neq \varnothing$. Now suppose $A$ has positive upper density. An elementary argument proves there is a Borel probability measure $\mu$ on $X_{A}$ such that (a) $\mu$ is $T$-invariant $\left(\mu\left(T^{-1} B\right)=\mu(B)\right.$ for every Borel set $\left.B\right)$, and (b) $\mu\left(U_{A}\right) \geqslant \alpha$, where $\alpha$ is as in the definition of "positive upper density". Therefore, Szemerédi's Theorem A follows from

TheOREM B (FurstenBERg [161]). Let $T$ be a measure preserving transformation of a measure space $(X, \circlearrowleft \mathcal{B}, \mu)$ with $\mu(X)<\infty$. For all $B \in \mathscr{B}$ and $k>0$, if $\mu(B)>0$, there exists an integer $n>0$ such that

$$
\mu\left(B \cap T^{n} B \cap \cdots \cap T^{(k-1) n} B\right)>0 .
$$

The proof of Theorem $B$ is rather long and difficult, involving in part a new structure theorem ${ }^{2}$ for measure preserving transformations of finite separable

2 ADDED IN PROOF. The structure theorm itself is due independently to R. J. Zimmer (preprint, U. S. Naval Academy). 
measure spaces. The latter may be viewed as an ergodic theoretic analogue of the structure theorem for minimal flows which will be proved in Part 2 of the present paper. As Furstenberg notes, Theorem B also follows from Theorem A by an application of the ergodic theorem.

REMARK. The easy case $k=2$ of Theorem B is the "Poincaré recurrence theorem," which is proved using the shoebox principle. The proof of van der Waerden's theorem makes use of the shoebox principle (see [164] for the easiest proof), while Szemerédi's proof of Theorem A, in turn, makes use of van der Waerden's theorem.

1.1. Uniformly distributed sequence generators. There exist sequences $\boldsymbol{R}$ $=\left(r_{1}, r_{2}, \ldots\right)$ of positive integers with the following universal property: If $G$ is any locally compact topological group and $\alpha=\left\{\alpha_{n} ; n \geqslant 1\right\}$ any sequence which generates a dense subgroup of $G$, then the sequence $\beta=\left\{\beta_{n}\right\}, \beta_{n}$ $=\alpha_{n_{1}} \alpha_{r_{2}} \cdots \alpha_{r_{n}}, n \geqslant 1$, is "uniformly distributed" in $G$, in the sense that

$$
\lim _{M \rightarrow \infty} M^{-1} \sum_{n=1}^{M} f\left(\beta_{n}\right)=\Re(f)
$$

exists for every continuous almost periodic or positive definite function on $G$ $(f \in \mathbb{Q}(G)$ or $\mathscr{P}(G))$. The limit (1.1.1) agrees on $\mathscr{Q}(G)$ with the Bohr-von Neumann mean for almost periodic functions and on $\mathscr{P}(G)$ with the Godement mean for positive definite functions [97], [57]. The construction of such uniformly distributed sequence generators $R$ (u.d.s.g.)'s was given, along with explicit examples, in [148], [140]. I shall indicate the ideas involved in the next two sections.

If $G$ is a compact group, then $\mathscr{Q}(G)=C(G)$, and the notion of uniform distribution given by (1.1.1) reduces to the generalization of Weyl's classical notion made by Eckmann [158], [30]. In this setting it is equivalent to require of $\beta$ that whenever $U \subseteq G$ is an open set whose boundary has measure 0 , $\lim _{M \rightarrow \infty} M^{-1} \sum_{n=1}^{M} \chi_{U}\left(\beta_{n}\right)$ exists and is the Haar measure of $U$, normalized so that $G$ has measure 1 .

Whether $G$ is compact or not our notion of uniform distribution is equivalent to a "Weyl's criterion".

\subsubsection{Proposition. A sequence $\beta$ is uniformly distributed in $G$ if and only if}

$$
\lim _{M \rightarrow \infty} \frac{1}{M} \sum_{n=1}^{M} \pi\left(\beta_{n}\right)=0
$$

in the weak operator topology for every continuous nontrivial irreducible unitary representation $\pi$ of $G$.

The proposition is proved by using von Neumann's approximation theorem for almost periodic functions and Godement's representation theorem (a generalization of Bochner's theorem) for positive definite functions [97], [57].

Other generalizations (with examples and/or constructions) of uniform distribution, which generally require that (1.1.3) hold when the kernel of $\pi$ has compact index (so that $\pi$ is necessarily finite dimensional), have been studied by Niven [98], Rubel [117], S. Hartman [60], Berg, Rajagopolan, and Rubel [11], and Benzinger [10]. Another notion, which requires (1.1.3) to hold in the 
strong operator topology, occurs in Rindler [114]. I do not know if u.d.s.g.'s exist for Rindler's notion.

The following theorem for $G=\mathbf{Z}$ corresponds to the classical theorem of Weyl [158] on the uniform distribution mod 1 of the fractional parts of real (irrational) polynomials. [] denotes the greatest integer function.

1.1.4. THEOREM [151]. Let $P(n)=a_{0}+a_{1} n+\cdots+a_{k} n^{k}$ be a polynomial with real coefficients. The sequence $\beta_{n}=[P(n)]$ is uniformly distributed in $\mathbf{Z}$ if and only if either (a) $k=1$ and $a_{1}=1 / q$ for some integer $q$, or (b) the coefficients $a_{1}, a_{2}, \ldots, a_{k}$ do not lie in a singly generated (additive) subgroup of R.

The proof of this theorem does not involve dynamics or ergodic theory. In the sense of Niven the sequence $[\alpha n+\beta]$ is uniformly distributed in $\mathbf{Z}$ whenever $\alpha$ is irrational [98], but in our sense it never is (by (a)).

REMARK. It is an open question, I believe, whether a group $G$ which contains a countable dense subset also contains a sequence $\beta$ which satisfies (1.1.1) for every continuous weakly almost periodic function on $G(f \in W(G))$. The question is meaningful because there does exist on $W(G)$ a unique "invariant mean" $\Re(\cdot)$, and $W(G)$ contains $A(G) \cup \mathscr{P}(G)$ [29], [27], [118]. Our construction of u.d.s.g.'s does imply

1.1.5. THEOREM. Let $G$ be a locally compact topological group. If $G$ contains a countable dense subset, $G$ contains a uniformly distributed sequence.

1.2. Weak mixing relative to a partition. In what follows $(X, \Re, \mu)$ denotes a probability space and $T$ a measure preserving transformation (endomorphism). That is, $T: X \rightarrow X$ is $\Re$-measurable, and $\mu\left(T^{-1} B\right)=\mu(B), B \in \mathscr{B}$. If $\mathscr{H}$ is a separable Hilbert space, $\mathfrak{A}(\mathcal{H})$ denotes the group of unitary operators on $\mathcal{H}$ with the weak operator topology. Given measurable functions $F: X$ $\rightarrow \mathfrak{A}(\mathfrak{H})$ and $h: X \rightarrow \mathfrak{H}$, define $V_{F} h(x)=F(x) h(T x)$. Finally, if $P$ is a partition of $X$ into a finite or countable number of measurable sets, define a function to be $P$-measurable if it is constant on the atoms of $P$.

1.2.1. Definition [140]. With notations as above, $T$ is weak mixing relative to $P$, or simply $P$-mixing if for any $\mathcal{H}$ and $P$-measurable $F: X \rightarrow \mathfrak{A}(\mathcal{H})$, every 9 measurable solution to $V_{F} h=h$ is essentially constant.

If we take $\mathcal{H}=\mathbf{C}$ and $F \equiv 1$, we see that $P$-mixing implies ergodicity $(h(T x)=h(x)$, a.e., implies $h$ is essentially constant). Also, if a general $F$ is multiplied by $\bar{\zeta},|\zeta|=1$, the $P$-mixing condition implies $V_{F} h=\zeta h$ has no nonconstant solutions. In particular (taking $\mathfrak{H}=\mathbf{C}$ and $F \equiv 1$ again), $T$ is weak mixing if it is $P$-mixing. The spectral theorem may be used to show that if $T$ is weak mixing, then $T$ is $P$-mixing for the trivial partition $P=\{X\}$.

1.2.2. Question. Do ergodicity or strong mixing $\left(\lim _{n \rightarrow \infty} \mu\left(A \cap T^{-n} B\right)\right.$ $=\mu(A) \mu(B)$ for all $A, B \in \mathscr{B})$ have relative-to- $P$ counterparts?

If $P$ is a partition, $T^{-n} P$ denotes the partition $T^{-n} P=\left\{T^{-n} A \mid A \in P\right\}$, and $P_{0}^{n}$ denotes the join $P_{0}^{n}=\vee_{k=0}^{n} T^{-k} P$. A typical element of $P_{0}^{n}$ is $A_{0} \cap T^{-1} A_{1} \cap \cdots \cap T^{-n} A_{n}, A_{j} \in P, 0 \leqslant j \leqslant n$. Thus, $P_{0}^{n}$ is at most countable, as is $\cup_{n \geqslant 0} P_{0}^{n}$.

1.2.3. Definition. A point $x \in X$ is generic relative to $(T, P)$, or simply $(T, P)$-generic, if for every $n$ and $B \in P_{0}^{n}$, 


$$
\lim _{M \rightarrow \infty} \frac{1}{M} \sum_{m=1}^{M} \chi_{B}\left(T^{m} x\right)=\mu(B) .
$$

If $T$ is ergodic (e.g., if $T$ is $P$-mixing), countably many applications of the ergodic theorem imply almost every point $x$ is $(T, P)$-generic.

The lemma to follow generalizes a principle of Furstenberg [49].

1.2.5. Lemma [148]. Let $x \in X$ be $(T, P)$-generic, and let $F: X \rightarrow \mathfrak{A}(\mathfrak{H})$ be $P$ measurable. Then either the equation $V_{F} h=h$ has a nontrivial (i.e., not essentially 0) measurable solution, or else

$$
\lim _{M \rightarrow \infty} \frac{1}{M} \sum_{n=1}^{M}\left(F(x) F(T x) \cdots F\left(T^{n-1} x\right)\right)=0
$$

in the weak operator topology.

The proof of the lemma involves only the definition of Hilbert space and the fact it is self-dual.

In what follows, $P$ is further restricted to have infinitely many atoms, each of positive measure, and we order $P$ in some way as $P=\left\{B_{1}, B_{2}, \ldots\right\}$. Define $r: X \rightarrow \mathbf{Z}^{+}$by $r=\sum_{k=1}^{\infty} k \chi_{B_{k}}$ (so that $r \equiv k$ on $B_{k}$ ). Finally, let $r_{n}(x)$ $=r\left(T^{n-1} x\right), n \geqslant 1$, and $R(x) \stackrel{B_{k}}{=}\left(r_{1}(x), r_{2}(x), \ldots\right)$.

1.2.7. Theorem [148], [140]. Assume $T$ is P-mixing. Then for every $(T, P)$ generic point $x, R(x)$ is a u.d.s.g.

SKETCH OF PROOF. Assume a sequence $\alpha$ generates a dense subgroup of $G$, let $\pi: G \rightarrow \mathfrak{U}(\mathcal{H C})$ be continuous and irreducible, and define $F \equiv \pi\left(\alpha_{k}\right)$ on $B_{k}$. Now for any $n \geqslant 1$,

$$
F(x) F(T x) \cdots F\left(T^{n-1} x\right)=\pi\left(\alpha_{r_{1}}\right) \pi\left(\alpha_{r_{2}}\right) \cdots \pi\left(\alpha_{r_{n}}\right)=\pi\left(\beta_{n}\right) .
$$

Therefore, if $x$ is $(T, P)$-generic, and if (1.1.3) fails, (1.2.5) also fails, and $V_{F} h=h$ has a nonzero solution. This solution must be constant a.e., say $h=w \in \mathcal{H}, w \neq 0$. Since $\mu\left(B_{k}\right)>0$, all $k, \pi\left(\alpha_{k}\right) w=w$, all $k$. As $\pi$ is a continuous representation and $\alpha$ generates a dense subgroup, $\pi(g) w=w, g$ $\in G$. That is, $\pi$ is trivial, and $\beta$ is uniformly distributed in $G$.

Remark. As remarked earlier, $(T, P)$-generic points exist when $T$ is $P$ mixing. The construction of u.d.s.g.'s is thereby reduced to the construction of $T$ and $P$ (as above) such that $T$ is $P$-mixing.

1.3. Construction of $P$-mixing transformations. For every integer $m>1$ let $S_{m}$ be the transformation of $I=[0,1)$ defined by $S_{m} x=m x(\bmod 1) . S_{m}$ preserves Lebesgue measure on $I$, and if $P_{m}$ is the partition $P_{m}=\{[j / m$, $(j+1) / m) \mid 0 \leqslant j \leqslant m\}$, the $\left(S_{m}, P_{m}\right)$-generic points are precisely the normal members to the base $m$ ( $m$-normal numbers). Almost every number is normal to every base (Borel [15]).

Examples of normal numbers exist. For example, if $m=10$,

$$
x=.12345678910111213 \ldots
$$

is normal (Champernowne [21]). For more examples see [25], [26]. 
If $S$ is an endomorphism of $(X, \mathscr{B}, \mu)$, and if $Y \subseteq X$ is a set of positive measure, the Poincaré recurrence theorem [59] implies that for almost all $y \in Y$ there exists $q>0$ with $S^{q} y \in Y$. Choosing $q$ as small as possible there is defined (a.e.) a map $T: Y \rightarrow Y, T y=S^{q} y$, called the induced transformation. $T$ preserves the measure $\mu$ normalized on (and restricted to) $Y$ (Kakutani [67]).

Now let $Y \subseteq I$ be an open set, and let $W=\partial Y$. Use $|\cdot|$ for Lebesgue measure, $W_{\varepsilon}$ for the $\varepsilon$-neighborhood of $W$, and define a number $\delta(W), 0$ $\leqslant \delta(W) \leqslant 1$, by

$$
\delta(W)=\liminf _{\varepsilon \rightarrow 0} \frac{\log \left|W_{\varepsilon}\right|}{\log \varepsilon} .
$$

(The number $1-\delta(W)$ is the "upper Minkowski dimension" of $W$.) Below, $T_{m}$ denotes the transformation induced on $Y$ by $S_{m}, P^{(m)}$ is the partition of $Y$ into sets for which $q$ (the first return time) $=1,2, \ldots$.

1.3.2. Lemma [148]. Assume $\delta(W)>0$, and let $T_{m}, P^{(m)}$ be as above. If $F: Y \rightarrow \mathfrak{H}(\mathcal{H})$ is $P^{(m)}$ measurable, then any measurable solution to $V_{F} h=h$ is essentially piecewise constant with finite range. In case either

(a) $Y=(a, b)$ with $(m, a, b) \neq\left(2, \frac{1}{6}, \frac{5}{6}\right)$ or $\left(2, \frac{1}{4}, \frac{3}{4}\right)$, or

(b) $Y \subseteq(0,1-1 / m)$ or $(1 / m, 1)$,

$h$ is essentially constant.

When $\delta(W)>0, W$ has measure 0 , and $\chi_{Y}$ is Riemann integrable. In this case every $m$-normal number in $Y$ is $\left(T_{m}, P^{(m)}\right)$ generic. In fact, if $x$ is any $m$ normal number, and if $S_{m}^{q} x \in Y$, then $S_{m}^{q} x$ is $\left(T_{m}, P^{(m)}\right)$ generic.

If $Y$ satisfies (a) or (b) of the lemma, then $T_{m}$ is $P^{(m)}$-mixing, and if $0<|Y|<1, P^{(m)}$ is essentially infinite. Thus, u.d.s.g.'s exist. In case $m=10$ and $Y=\left(\frac{3}{10}, \frac{4}{10}\right)$, say, $P^{(10)}$ is the partition into sets $\{q=1\},\{q=2\}, \ldots$ If $x$ is as in (1.3.1), then $S_{m}^{2} x \in Y$, and $R\left(S_{m}^{2} x\right)$ is the sequence $r_{1}=14, r_{2}=20$, .... Thus, we have a concrete u.d.s.g.

1.4. Well distributed sequences. Hlawka [65] has introduced the notion of a well distributed sequence in a compact group. The extension of the notion to arbitrary topological groups $G$ is as follows: we say $\beta=\left\{\beta_{n}\right\}$ is well distributed in $G$ if for all $f \in \mathbb{Q}(G) \cup \mathscr{P}(G)$,

$$
\lim _{M \rightarrow \infty} \frac{1}{M} \sum_{n=1}^{M} f\left(\beta_{n+k}\right)=\Re(f)
$$

holds uniformly in $k \geqslant 0$.

If $G$ is a compact group which contains a countable dense subset, then according to Rindler [114], [115], $G$ contains a well distributed sequence. The corresponding result for noncompact $G$ seems to be open. In particular, it is open whether "well distributed sequence generators" (w.d.s.g.'s) exist (the definition is obvious). I shall make a few remarks on the problem.

Call the point $x$ in Definition 1.2.3 $(T, P)$-strictly generic if when $T^{m} x$ is replaced by $T^{m+k} x$, (1.2.4) holds uniformly in $k \geqslant 0$. Now the analogue of Theorem 1.2.7 is true: If $T$ is $P$-mixing, and if $x$ is $(T, P)$-strictly generic, then $R(x)$ is a w.d.s.g. This is essentially proved in [148]. The problem now is that in the known examples, e.g., $\$ 1.3$ and [148], [140], there are no strictly generic 
points. On the other hand, in certain settings where there are strictly generic points, weak mixing and, hence, $P$-mixing fail $[140]$.

1.4.2. Definition. [86], [100]. Let $X$ be a compact metric space and $T: X \rightarrow X$ a continuous map. The "cascade" $\mathcal{X}=(T, X)$ is uniquely ergodic if there exists a unique Borel probability measure on $X$ which is invariant under $T$.

Invariant measures will always exist [86], and therefore the important condition in the definition is the uniqueness. If $\mathcal{X}$ is uniquely ergodic, and if $\mu$ is the unique measure of the definition, then $T$ is ergodic relative to $\mu$ [100]. It follows from the work of Oxtoby [100] that if $U$ is an open set with $\mu(\partial U)=0$, then all points $x$ are $\left(T,\left\{U, U^{c}\right\}\right)$ strictly generic. If $\mu$ is not atomic, there will exist an infinite partition $P$, all of whose atoms have positive measure, with the property that all points are $(T, P)$-strictly generic.

1.4.3. Question. Is it possible to find $\mathfrak{X}$ uniquely ergodic and $P$ as above such that $T$ is $P$-mixing?

Recall that $T$ is weak mixing if it is $P$-mixing, and therefore $\mathscr{X}$ must be rejected if $T$ is not weak mixing relative to $\mu$ (or if $\mu$ is a point mass; i.e., if $T$ has a stationary point). In this regard there is a remarkable theorem due to $\mathrm{R}$. Jewett:

1.4.4. THEOREM (R. JEWETT [66]) . Let $T_{0}$ be a weakly mixing automorphism of a Lebesgue probability space $(Y, \Im, \nu)$. There exists a uniquely ergodic cascade $\mathcal{X}=(T, X)$, with $X$ the Cantor set, and an invertible measurable map $\varphi: Y \rightarrow X$ such that $\varphi T_{0}=T \varphi$ a.e.

If we define $\varphi \nu(A)=\nu\left(\varphi^{-1} A\right)$ for every Borel set $A \subseteq X$, then $\varphi \nu=\mu$ is the unique invariant probability measure for $T$. Therefore $T$ is weak mixing relative to $\mu$.

Jewett's theorem was extended to ergodic $T_{0}$ by Krieger [85], to weak mixing one parameter flows by Jacobs [65], and to ergodic flows by Denker and Eberlein [28]. See also [84] for more references.

It is probable that w.d.s.g.'s can be constructed using certain nonweak mixing transformations. For example, let $\theta \in \mathbf{R}$ be irrational, and define $T x=x+\theta(\bmod 1), x \in[0,1)$. Take $P$ to be a finite partition $P=\left\{\left[t_{j-1}, t_{j}\right) \mid\right.$ $1 \leqslant j \leqslant n\}$, where $0=t_{0}<t_{1}<\cdots<t_{n}=1$. I conjecture that if the $t_{j}$ 's are rational, then, for all $x, R(x)$ is a w.d.s.g. Since $R(x)$ assumes only values $1,2, \ldots, n$, "w.d.s.g." is in the sense that if $G$ contains $\alpha=\left\{\alpha_{1}, \ldots, \alpha_{n}\right\}$ which generate a dense subgroup, the associated sequence $\beta$ is well distributed in $G$. Thus far the conjecture has been verified for finite groups $G$ under an additional assumption on $\theta([\mathbf{1 4 2}]$ that it have bounded partial quotients in its continued fraction expansion).

For example, if $G_{k}$ is the permutation group on $k$ letters, $G_{k}$ is generated by $\alpha_{-1}=(12)$ and $\alpha_{1}=(23 \cdots k 1)$. If we take $n=2, t_{1}=\frac{1}{2}$, and $\theta=\sqrt{ } 5$, the result mentioned above implies that the sequence $\beta_{l}=\prod_{j=1}^{l} \alpha_{\mathrm{sgn} \cos 2 \pi j \sqrt{ } 5}$ is well distributed in $G_{k}$. I do not know a direct proof of this fact.

1.5. A theorem in Diophantine approximation. Let $\Sigma=\left\{\sigma_{1}<\sigma_{2}<\cdots\right\}$ be an increasing sequence of integers. A theorem of Weyl implies that, for almost all $x, \Sigma x$ is uniformly distributed modulo 1 , where $\Sigma x=\left\{\sigma_{n} x\right\}$. On the other 
hand, given any irrational number $x$, one easily constructs $\Sigma$ with bounded gaps such that $\Sigma x$ is not even dense modulo 1 (e.g., $\left.\sigma_{n} x \in\left(0, \frac{1}{2}\right) \bmod 1\right)$. However, if $\Sigma$ is a multiplicative semigroup which is not lacunary, that is, $\Sigma$ is not contained in the set of powers of a single integer, the situation regarding density is as nice as possible.

1.5.1. THeOREM (FURSTENBERG [48]). Let $\Sigma$ be a nonlacunary semigroup of positive integers. For every irrational number $x$ the sequence $\Sigma x$ is dense modulo 1.

If $\Sigma$ is lacunary, it is easy to see that the set of $x$ such that $\Sigma x$ is not dense has Hausdorff dimension 1 (but measure 0 ). In the case of such sparse, but nonlacunary, semigroups as $\Sigma=\left\{\left(2^{n} 3^{m}\right) \mid n, m \geqslant 0\right\}$, for which the theorem was new, it was noted in [48] that there exist irrational $x$ such that $\Sigma x$ is not uniformly distributed.

I shall outline Furstenberg's proof in the next two sections, partly for the purpose of describing his notion of "disjointness" in topological dynamics.

1.6. Disjointness in topological dynamics. In what follows $\mathfrak{X}=(\Sigma, X)$, $\mathscr{Y}$ $=(\Sigma, Y), \ldots$ will denote flows; i.e., actions of a fixed semigroup $\Sigma$ (the phase semigroup) by continuous transformations of compact metric spaces $X, Y, \ldots$ (the phase spaces). The image of $x \in X, y \in Y, \ldots$ under $\sigma \in \Sigma$ is denoted $\sigma x, \sigma y, \ldots$

Given flows $\mathfrak{X}_{j}=\left(\Sigma, X_{j}\right), j=1,2$, the product $\mathfrak{X}_{1} \times \mathfrak{X}_{2}=\left(\Sigma, X_{1} \times X_{2}\right)$ is defined by $\sigma\left(x_{1}, x_{2}\right)=\left(\sigma x_{1}, \sigma x_{2}\right)$. A homomorphism $\mathcal{X} \rightarrow^{\pi}$ Oु is a continuous, equivariant $(\pi \sigma=\sigma \pi, \sigma \in \Sigma)$, surjective map $\pi: X \rightarrow Y$. $\mathcal{Y}$ is said to be a

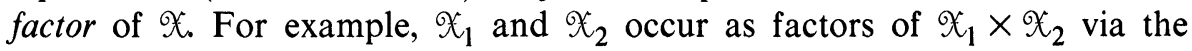
projections onto the respective coordinates.

$\mathcal{X}$ is a minimal flow if the phase space contains no proper closed invariant set, or equivalently, if every orbit $(\Sigma x)$ is dense. It is straightforward to prove that any factor of a minimal flow is minimal. On the other hand, the product of two minimal flows is not usually minimal. For example, in $\mathfrak{X} \times \mathfrak{X}$ the diagonal is a closed invariant set which is proper if $\mathscr{X}$ is not trivial.

1.6.2. Definition (Furstenberg [48]). $\mathscr{X}_{1}$ and $\mathfrak{X}_{2}$ are disjoint flows if for every pair of homomorphisms $\mathfrak{X} \rightarrow \pi_{j} \mathfrak{X}_{j}, j=1,2$, the map $\pi_{1} \times \pi_{2}$, defined by $\pi_{1} \times \pi_{2}(x)=\left(\pi_{1}(x), \pi_{2}(x)\right)$, is also a homomorphism (i.e., surjective).

A useful "internal" criterion for disjointness is contained in the following elementary proposition.

1.6.3. Proposition [48]. $\mathfrak{X}_{1}$ and $\mathfrak{X}_{2}$ are disjoint flows if and only if no proper closed invariant subset of $X_{1} \times X_{2}$ projects onto $X_{1}, X_{2}$ under the coordinate maps.

The proposition implies disjoint flows are relatively prime, i.e., have no common factors. For if $\mathfrak{X}_{1}, \mathfrak{X}_{2}$ are disjoint, and if $\mathfrak{X}_{j} \rightarrow \pi_{j} \mathscr{Y}, j=1,2$, are homomorphisms, define $\Delta \subseteq X_{1} \times X_{2}$ to be the set of $\left(x_{1}, x_{2}\right)$ with $\pi_{1} x_{1}$ $=\pi_{2} x_{2} . \Delta$ is closed and invariant and projects onto $X_{1}, X_{2}$ under the coordinate maps. Therefore $\Delta=X_{1} \times X_{2}$, and Oु is trivial.

Relatively prime flows are not necessarily disjoint, and therefore there is no "Chinese Remainder Theorem" for flows. (The reader will find it instructive to verify that the Chinese Remainder Theorem asserts that the natural flows 
$(\mathbf{Z}, \mathbf{Z} / a \mathbf{Z}),(\mathbf{Z}, \mathbf{Z} / b \mathbf{Z}), a, b \in \mathbf{Z}-\{0\}$, are disjoint if they are relatively prime, i.e., $(a, b)=1$.) For one thing, if $\mathfrak{X}_{1}, \mathfrak{X}_{2}$ are disjoint, at least one must be minimal. But even when both are minimal, disjointness does not follow from being relatively prime [83]. However, it is an open question whether relatively prime minimal flows with abelian phase semigroup are disjoint, or, equivalently, if their product is minimal.

A flow $\mathcal{X}$ is topologically transitive if every proper closed invariant set has empty interior. If $\Sigma$ is a group, topological transitivity is equivalent to the existence of a dense orbit (Hedlund [63]) and in any case implies the set of $x \in X$ with dense orbits is residual.

1.6.5. Definition [48]. $\mathcal{X}$ is an $F$-flow if there exists a sequence $\left\{F_{n}\right\}$ of finite subsets of $X$ such that (a) $\cup_{n} F_{n}$ is dense, (b) $F_{n}$ is invariant, and each $\sigma \in \Sigma$ is one-to-one on $F_{n}$, and (c) if $\Sigma^{(n)}=\left\{\sigma \in \Sigma \mid \sigma x=x, x \in F_{n}\right\}$, then $\mathcal{X}^{(n)}$ $=\left(\Sigma^{(n)}, X\right)$ is topologically transitive.

One can prove every factor of an $F$-flow is an $F$-flow and every minimal $F$ flow is trivial. 'Therefore, every $F$-flow is relatively prime to every minimal flow. In this case disjointness is implied when $\Sigma$ is abelian:

1.6.6. THEOREM [48]. Let $\mathfrak{X}_{1}$ and $\mathfrak{X}_{2}$ be a minimal flow and an F-flow, respectively. If $\Sigma$ is abelian, $\mathfrak{X}_{1}$ and $\mathfrak{X}_{2}$ are disjoint.

SKetch OF PROOF. Let $\Delta \subseteq X_{1} \times X_{2}$ be a closed invariant set which projects onto $X_{1}, X_{2}$ under the coordinate maps. By Proposition 1.6.3, it will suffice to prove $\Delta=X_{1} \times X_{2}$, and this will follow if we prove $X_{1} \times F_{n} \subseteq \Delta$ for all $n$ (by (a)). To this end, fix $n$ and choose a $\Sigma^{(n)}$ minimal set $M \subseteq X_{1}$ (nonempty, closed, $\Sigma^{(n)}$ invariant and minimal with respect to these properties; use Zorn's lemma). As $\Sigma$ is abelian, $\sigma M$ is $\Sigma^{(n)}$ minimal for all $\sigma \in \Sigma$. Moreover, if $\left.\sigma\right|_{F_{n}}=\left.\tau\right|_{F_{n}}, \sigma M=\tau M$. (If $\tau^{l}=$ id on $F_{n}, \sigma \tau^{l} \in \sigma \Sigma^{(n)} \cap \tau \Sigma^{(n)}$, and so $\sigma M$ $\cap \tau M \neq \varnothing$. Minimality under $\Sigma^{(n)}$ implies they are equal.) It follows that if $\sigma_{1}, \ldots, \sigma_{k}$ is a complete set of representatives for $\left.\Sigma\right|_{F_{n}}, \cup_{j=1}^{k} \sigma_{j} M$ is $\Sigma$ invariant, hence all of $X_{1}$ (as $\mathfrak{X}_{1}$ is minimal). Now to prove $X_{1} \times F_{n} \subseteq \Delta$, it is enough to prove $M \times F_{n} \subseteq \Delta$, since $\cup_{j=1}^{k} \sigma_{j}\left(M \times F_{n}\right)=X_{1} \times F_{n}$. To this end, choose $x_{2} \in X_{2}$ with dense $\Sigma^{(n)}$ orbit (by (c)). For some $x_{1} \in X_{1},\left(x_{1}, x_{2}\right) \in \Delta$ by hypothesis, and if, say, $x_{1} \in \sigma_{j} M, \Sigma^{(n)}\left(x_{1}, x_{2}\right) \subseteq \Delta \cap\left(\sigma_{j} M \times X_{2}\right)$. Denoting its closure by $\Delta^{\prime}$, the choice of $x_{2}$ implies $\Delta^{\prime}$ projects onto $X_{2}$ in the second coordinate. Now choose $\sigma$ so that $\left.\sigma \sigma_{j}\right|_{F_{n}}=$ id. Then $\Delta^{\prime \prime}=\sigma \sigma_{j} \Delta^{\prime} \subseteq\left(M \times X_{2}\right)$ $\cap \Delta$, and as $\sigma X_{2}=X_{2}$ (by (a) and (b)), $\Delta^{\prime \prime}$ also projects onto $X_{2}$ in the second coordinate. Therefore, if $y \in F_{n}$, there exists $x \in M$ such that $(x, y) \in \Delta$. Since $\Sigma^{(n)} x$ is dense in $M$ and $\Sigma^{(n)} y=\{y\}, M \times\{y\} \subseteq \Delta$. Therefore, $M \times F_{n}$ $\subseteq \Delta$.

1.7. Proof of Theorem 1.5.1 [48]. If $\Sigma$ is a multiplicative semigroup of positive integers, there is a natural flow $x=(\Sigma, X), X=[0,1)$, defined by $x \rightarrow \sigma x(\bmod 1)$. If $\Sigma \neq\{1\}$, almost every point has a dense orbit, and therefore $\mathfrak{X}$ is topologically transitive. Further, if there exists a prime $p$ such that $(p, \sigma)=1, \sigma \in \Sigma$, the sets $F_{n}=\left\{j / p^{n} \mid 0 \leqslant j<p^{n}\right\}$ enjoy properties (a) and (b) of the definition of an $F$-flow, and therefore $x$ is an $F$-flow. If no such prime exists, replace $\Sigma$ by a smaller semigroup for which one does. This can be done without destroying nonlacunarity. 
If $\Sigma=\left\{\sigma_{1}<\sigma_{2}<\cdots\right\}$ is nonlacunary, it can be shown that $\lim _{n \rightarrow \infty}\left(\sigma_{n} / \sigma_{n+1}\right)=1$, and this readily implies that if a closed invariant set $A$ has a rational cluster point, then $A=X$. In particular, if $B$ is closed, invariant, and infinite, $A=B-B$ is closed, invariant, and has 0 as a cluster point. Therefore $B-B=X$.

Now let $x \in X$ be irrational, and let $Y=\overline{\Sigma x}$. In $Y$ we choose a $\Sigma$ minimal set $M$. If $M$ is finite, $\Sigma$ is transitive on $M$, and this implies $M$ consists of rationals. These must occur as cluster points of $Y$, and therefore $Y=X$. If $M$ is infinite, then by the above, $M-M=X$. But this cannot happen. In fact,

1.7.1. Proposition [48]. With notations as above, suppose $M$ is a $\Sigma$ minimal set and $B$ a closed invariant set such that $M-B=X$. Then $B=X$.

Proof. $\Re=(\Sigma, M)$ and $\mathcal{X}$ are disjoint by Theorem 1.6.6. Let $Z=M \times B$ and $\mathscr{Z}=(\Sigma, Z)$ be natural. The maps $\pi_{1}(m, b)=m$ and $\pi_{2}(m, b)=m-b$ define homomorphisms $\mathscr{Z} \rightarrow \pi^{\pi_{j}} \mathfrak{X}$. By disjointness $\pi_{1} \times \pi_{2}(\mathbf{Z})=\{(m, m-b) \mid$ $m \in M, b \in B\}=M \times X$, and this clearly implies $B=X$.

In the special case $B=M$ above, $M=X$ is impossible (because 0 is not minimal). Thus, $M$ is finite, and Theorem 1.5 .1 is proved.

1.8. Quasiregular points and generic points. In this section I shall describe some recent work on generic points for cascades $x=(T, X)(\S 1.4)$, some of which will also play an important role in later sections. The notion of genericity is slightly different from, but in the same spirit as, the notion in earlier sections.

Call a point $x \in X$ quasiregular for $\mathcal{X}$ if for all $f \in C(X)$ the limit

$$
\lim _{M \rightarrow \infty} \frac{1}{M} \sum_{n=1}^{M} f\left(T^{n} x\right)=\Lambda_{x}(f)
$$

exists (Krylov-Bogoliouboff [86]). If $\mu \in \mathscr{P}_{I}(\stackrel{(N)}{)}$, the set of invariant Borel probability measures on $X$, then $\mu$ almost all points are quasiregular. This is proved by applying the ergodic theorem to a countable dense subset of $C(X)$. Since $\mathscr{P}_{I}(\mathcal{X}) \neq \varnothing$, quasiregular points exist.

To each quasiregular point $x$ there corresponds a unique $\mu=\mu_{x} \in Q_{I}$ such that

$$
\Lambda_{x}(f)=\int_{X} f(y) \mu_{x}(d y) \quad(f \in C(X)) .
$$

We sometimes write $\Lambda_{x}=\Lambda_{x}^{\mu}$.

A point $x$ is generic for $\mu \in \mathscr{P}_{I}$ if $x$ is quasiregular, and $\Lambda_{x}=\Lambda_{x}^{\mu}$. Every quasiregular point is generic for some $\mu$, and if $\mu$ is ergodic ( $\equiv$ an extreme point of the convex set $\left.\varphi_{I}[13]\right), \mu$-almost all points are generic. If $\mu$ is not ergodic, the set of $\mu$-generic points has $\mu$-measure 0 [101] and may even be empty (as for example happens when $T$ is the identity).

If $X=[0,1)$ and $T x=r x(\bmod 1)$ for some $r>1$, then every $\mu \in$ oP $_{I}$ possesses generic points (Ville [152]). In fact, Colebrook [23] has proved that if $\mu \in \mathscr{P}_{l}$, the Hausdorff dimension of the set of $\mu$ generic points is $h_{\mu}(T) / \log r$, where $h_{\mu}(T)$ is the entropy of $T$ with respect to $\mu$ (defined in a later section). See also Volkmann [153]. A theorem of Parry [102] implies this 
dimension is 1 only if $\mu$ is Lebesgue measure (and the $\mu$-generic points are the $r$-normal numbers).

Now let $\Omega$ be a compact metric space, and let $X=\Omega^{N}$ or $\Omega^{\mathbf{Z}}(N$ $=\{1,2, \ldots\})$. The left shift is defined by $(T x)_{n}=x_{n+1}, x=\left\{x_{n}\right\}$. In this case Kakutani has proved each $\mu \in \mathscr{P}_{I}$ has generic points (Oxtoby [101]).

If $x \in X, q \geqslant 1$, define $\mu_{x}^{(q)}$ to be the average of the (Dirac) point masses at $x, T x, \ldots, T^{q-1} x$. To say $x$ is generic for $\mu$ is to say $\lim _{q \rightarrow \infty} \mu_{x}^{(q)}=\mu$ in the weak-* topology. To prove Kakutani's theorem let $\mu \in \mathcal{P}_{I}(\mathfrak{X})$. By a theorem of Parthasarathy [103], [101] there exists a sequence $x_{k}$ of periodic points, $T^{q_{k}} x_{k}=x_{k}$, such that the (invariant) measures $\mu_{x_{k}}^{\left(q_{k}\right)}$ converge to $\mu$. A $\mu$ generic point $x$ is constructed by letting $x$ agree with $x_{1}$ in coordinates $1, \ldots, t_{1}$, with $x_{2}$ in $t_{1}+1, \ldots, t_{1}+t_{2}$, etc., where $t_{1}, t_{2}, \ldots$ are appropriately chosen (large multiples of $q_{1}, q_{2}, \ldots$ ). To discuss generalizations, a version of Bowen's notion of "specification" is necessary (Bowen [18]-[20]). The definition below is taken from Sigmund [133]. ox denotes a cascade and $d(\cdot, \cdot)$ a metric for $X$.

1.8.3. Definition. $\mathcal{X}$ satisfies specification if for every $\varepsilon>0$ there exists $M(\varepsilon)<\infty$ such that the following is true: if $x_{1}, x_{2} \in X,\left[a_{j}, b_{j}\right] \subseteq \mathbf{Z}$ (or $N$ ), $j=1,2$, and $p$ are such that (i) $a_{2}-b_{1}>M(\varepsilon)$, and (ii) $p>b_{2}-a_{1}+M(\varepsilon)$, then there is a point $x$ with period $p$ such that for all $l \in\left[a_{j}, b_{j}\right], j=1,2$, $d\left(T^{l} x, T^{l} x_{j}\right)<\varepsilon$.

Examples of cascades which satisfy specification are the left shift (obvious) and a hyperbolic automorphism of the $n$-torus (not obvious), i.e., one whose associated $n \times n$ integer matrix has no eigenvalues on the unit circle. For discussion of these other examples, see [19], [133].

Let $\mathcal{X}$ be any cascade. $\mathscr{P}_{I}(\mathcal{X})$ is weak-* compact, and therefore if $x \in X$, the set, $V(x)$, of weak-* cluster points of the sequence $\left\{\mu_{x}^{(q)} \mid q \geqslant 1\right\}$ is a nonempty compact subset of $\mathcal{P P}_{I} . V(x)$ may also be seen to be connected. Of course, $x$ is quasiregular precisely when $V(x)$ is a singleton.

I now state a theorem of Sigmund, a special case of which was proved (with a quantitative statement) by Colebrook [23]. See also Eggleston [31].

1.8.4. Theorem (K. Sigmund [133]). Assume satisfies specification. If $V \subseteq P_{I}$ is nonempty, closed, and connected, there exists $x \in X$ such that $V(x)=V$.

For the proof one makes use of the fact that the set $\left\{\mu_{x}^{(q)} \mid T^{q} x=x, q \geqslant 1\right\}$ is dense in ${ }^{\circ} P_{I}$ [132], which generalizes the Parthasarathy theorem, to construct a sequence $x_{k}, q_{k}, T^{q_{k}} x_{k}=x_{k}$ and weak-* neighborhoods (in $\left.\varphi_{I}\right) W_{k}$ of $\mu_{x_{k}}^{\left(q_{k}\right)}$ such that (a) $V$ is the set of cluster points of $\left\{\left(\mu_{x_{k}}^{\left(q_{k}\right)}\right) \mid k \geqslant 1\right\}$, (b) $W_{k} \cap W_{k+1}$ $\neq \varnothing$, all $k$ (this uses connectivity of $V$ ), and (c) $W_{k}-\mu_{x_{k}}^{\left(q_{k}\right)}$ "converges" to 0 . Now if $\left[a_{k}, b_{k}\right], k \geqslant 1$, are suitably chosen, the specification property may be used to prove that there exists $x \in X$ such that

$$
\lim _{k \rightarrow \infty} \max _{a_{k} \leqslant l \leqslant b_{k}} d\left(T^{l} x, T^{l} x_{k}\right)=0
$$

("orbit specification lemma" [133]). For this $x$ one uses (a)-(c) to prove $V(x) \subseteq V$ and if certain additional conditions are satisfied (e.g., the sequences $a_{k} / b_{k},\left(a_{k+1}-b_{k}\right) /\left(b_{k}-a_{k}\right)$, and $\left(q_{k-1}+q_{k}\right) /\left(b_{k-1}-a_{k-1}\right)$ converge to 0$)$, 
then $V(x) \supseteq V$. (If each $q_{k}$ is taken to be prime, then $V_{l}(x)=V, l \geqslant 1$, where $V_{l}(x)$ is formed from $\left(T^{l}, X\right)[\mathbf{2 3}],[\mathbf{1 3 3}]$.)

If $\mathcal{X}$ satisfies specification, then $X$ contains a dense set of periodic points, and $\mathfrak{X}$ may be seen to be topologically strongly mixing ([133]; if $U, V \neq \varnothing$ are open, then $U \cap T^{-n} V \neq \varnothing$ for all large $n$ ). The second property implies for each $l>0$ that $T^{l}$ is topologically transitive, and therefore $\mathcal{X}$ is an $F$-flow (§1.6). However, Sigmund's theorem is not true for $F$-flows. Weiss has constructed an $F$-flow $\mathcal{X}$ and nonempty open sets $V_{n} \subseteq X, n \geqslant 0$, with the property that for any $x$ if $\left\{T^{m} x\right\}$ visits each $V_{n}, n \geqslant 1$, with positive frequency, it visits $V_{0}$ with 0 frequency [157]. If $\left\{x_{k}\right\}$ is a dense set of periodic points with periods $\left\{q_{k}\right\}$, then $\mu=\sum_{k=1}^{\infty} 2^{-k-1} \mu_{x_{k}}^{\left(q_{k}\right)} \in \mathscr{P}_{I}$ assigns positive measure to every nonempty open set. Thus, $\mu$ has no generic point. (Also, there is no ergodic measure which is positive on nonempty open sets.)

1.8.5. Question. Do the quantitative theorems of Colebrook have generalizations; for example to hyperbolic automorphisms of the torus or to subshifts of finite type [102]?

Kamae [68] has generalized the Ville-Kakutani theorem in a direction which has deep applications. Let $\mathscr{X}$ be a cascade, $S \subseteq N$ an infinite set, and let $V^{S}(x)$ be the set of weak-* cluster points of $\left\{\mu_{x}^{(n)} \mid n \in S\right\}$. If $S=N, V^{S}=V$, and in any case $V^{S} \subseteq V$. Denote by $\Gamma_{x} \subseteq 2^{N}$ the set of $S$ such that $V^{S}(x)$ is a singleton, i.e., $\lim _{n \rightarrow \infty ; n \in S} \mu_{x}^{(n)}$ exists, and let $\mu_{x}^{S}$ be the limit.

In what follows $\mathfrak{X}_{j}$ is the left shift on $X_{j}=\Omega_{j}^{N}, j=1,2$, and if $\nu \in \mathscr{P}_{I}(\mathscr{X})$, $\mathfrak{X}=\mathfrak{X}_{1} \times \mathfrak{X}_{2},\left.\nu\right|_{X_{j}}$ denotes the projection of $\nu$ on $X_{j}, j=1,2$.

1.8.6. THEOREM (KAMAE [68]). With notations as above, let $\nu \in \mathscr{P}_{I}(\mathcal{X})$ and $\beta \in X_{2}$ be such that $\mu_{\beta}^{S}=\left.\nu\right|_{X_{2}}$ for some $S \in \Gamma_{\beta}$. There exists $\alpha \in X_{1}$ such that $S \in \Gamma_{(\alpha, \beta)}$ and $\mu_{(\alpha, \beta)}^{S}=\nu$. If $\left.\nu\right|_{X_{1}}$ is ergodic, $\alpha$ may be chosen generic for it.

When $X_{2}$ is trivial and $S=N$, the theorem reduces to the Ville-Kakutani theorem. In his proof Kamae employs a separate result, which I shall not state, to derive the second statement from the first. Below is a sketch of the ideas in the proof of the first part.

The basic idea is to construct a sequence $\left\{\alpha_{k}\right\} \subseteq X_{1}$ with the property that if $W$ is any neighborhood of $\nu$, then $V^{S}\left(\left(\alpha_{k}, \beta\right)\right) \subseteq W$ for large $k$. Then, as in previous arguments, $\alpha$ is built up from $\left\{\alpha_{k}\right\}$ by letting it agree with $\alpha_{1}$ on $\left[1, t_{1}\right), \alpha_{2}$ on $\left[t_{1}, t_{2}\right)$, etc. The essential point of the construction of $\left\{\alpha_{m}\right\}$ lies in finding, for one fixed $W$, a point $\alpha$ with $V^{S}((\alpha, \beta)) \subseteq W$. It is convenient (and no loss) to assume $W$ has the form $W=\left\{v^{\prime} \in \mathscr{P}_{I}||\left(F, v^{\prime}\right)-(F, v) \mid<\varepsilon\right\}$, where $F$ is continuous on $X_{1} \times X_{2}$ with values in $\mathbf{R}^{l}$, some $l>0$, and $(\cdot, \cdot)$ denotes bilinear pairing of measures and functions. It may further be assumed that $F(x, y)=F\left(\left(x_{1}, \ldots, x_{q}\right),\left(y_{1}, \ldots, y_{q}\right)\right)$ depends only upon the first $q$ coordinates. Now $\alpha$ will satisfy $V^{S}((\alpha, \beta)) \subseteq W$ if and only if

$$
\limsup _{n \rightarrow \infty ; n \in S}\left|\left(F, \mu_{(\alpha, \beta)}^{(n)}\right)-(F, \nu)\right|<\varepsilon .
$$

Kamae constructs a probability measure $\lambda$ on $X_{1}$ (not invariant) with the property that (1.8.7) holds for $\lambda$-almost all $\alpha$. The essential difficulties are already present when $\Omega_{2}$ is finite, which I now assume. 
To construct $\lambda$, auxiliary numbers $L \gg q$ and $M=R L \gg L$ are chosen to depend upon $q$ and $\varepsilon$. The coordinate functions on $X_{1}, X_{2}$ are denoted $X_{n}^{(j)}, j=1,2$, starting from the zeroth $n=0 . \mathscr{B}_{L}=\mathscr{B}_{(}\left(X_{0}^{(2)}, X_{1}^{(2)}, \ldots, X_{L-1}^{(2)}\right)$, the $\sigma$-algebra of subsets of $X_{2}$ generated by the first $L$ coordinate functions, may be regarded in a natural way as a $\sigma$-algebra in $X_{1} \times X_{2}$. In particular, the conditional expectation $E\left(\cdot \mid \Im_{L}\right)$ on $X_{1} \times X_{2}$ (using $\nu$ ) is defined, but may also be regarded as a $\mathscr{P}_{L}$ measurable function on $X_{2}$. Now define

$$
F_{i}(x)=F\left(T^{i}(x, \beta)\right), \quad x \in X_{1}, i \geqslant 0 .
$$

The measure $\lambda$ will be constructed with the properties (1) if $i \neq j$, the $M$-tuples $\left(X_{i M}^{(1)}, \ldots, X_{i M+M-1}^{(1)}\right)$ and $\left(X_{j M}^{(1)}, \ldots, X_{j M+M-1}^{(1)}\right)$ are independent, and (2)

$$
\left|\int_{X_{1}} F_{i} \lambda-\frac{1}{(L-q+1)} \sum_{t=0}^{L-q} E\left(F \circ T^{t} \mid \Re_{L}\right)\left(T^{i-t} \beta\right)\right|<\frac{\varepsilon}{3}
$$

whenever $L-1 \leqslant i-[i / M] M \leqslant M-L-q$. If $0 \leqslant t \leqslant L-q$, the facts $\mu_{\beta}^{S}=\left.\nu\right|_{X_{2}}$ and $\nu \in \mathscr{P}_{I}(\mathfrak{X})$ imply

$$
\begin{aligned}
& \lim _{\substack{n \rightarrow \infty \\
n \in S}} \frac{1}{n} \sum_{i=t}^{n} E\left(F \circ T^{t} \mid \Re_{L}\right)\left(T^{i-t} \beta\right)=\int_{X_{2}} E\left(F \circ T^{t} \mid \Re_{L}\right) \mu_{\beta}^{S} \\
&=\int_{X_{1} \times X_{2}} E\left(F \circ T^{t} \mid \Re_{L}\right) \nu=\int_{X_{1} \times X_{2}} F \circ T^{t} \nu:=\int_{X_{1} \times X_{2}} F \nu .
\end{aligned}
$$

It can be assumed the set of $i$ for which (2) holds has density at least $1-\varepsilon / 3$, and that $\|F\| \leqslant 1$, and therefore by $(1.8 .8)-(1.8 .9)$

$$
\limsup _{\substack{n \rightarrow \infty \\ n \in S}}\left|\frac{1}{n} \sum_{i=0}^{n-1} \int_{X_{1}} F_{i} \lambda-\int_{X_{1} \times X_{2}} F \nu\right|<\varepsilon .
$$

By the independence assumption (1) and the strong law of large numbers, $\lim _{n \rightarrow \infty} n^{-1} \sum_{i=0}^{n-1}\left(F_{i}-\int_{X_{1}} F_{i} \lambda\right)=0$, and since $F_{i}(\alpha)=F\left(T^{i}(\alpha, \beta)\right)$, it follows that (1.8.7) holds for $\lambda$-almost all $\alpha \in X_{1}$.

To construct $\lambda$, view $\Omega_{1}^{N}$ as a product of "clumps" $\Omega_{1}^{N}=\Omega_{1}^{M} \times \Omega_{1}^{M} \times \cdots$, and define $\lambda=\eta_{0} \times \eta_{1} \times \cdots$ as a product of measures defined on the clumps. (This makes the independence condition automatic.) Now $\eta_{k}$ is constructed as an average of $L$ measures $\sigma_{t}^{k}, 0 \leqslant t<L$, where for each $t$, $\sigma_{t}^{k}$ is constructed by viewing $\Omega_{1}^{M}$ as a product of $R+1$ clumps $(R L=M)$,

$$
\Omega_{1}^{M}=\Omega_{1}^{t} \times \frac{\Omega_{1}^{L} \times \cdots \times \Omega_{1}^{L}}{R-1} \times \Omega_{1}^{L-t},
$$

and writing $\sigma_{t}^{k}=\tau_{0} \times \tau_{1} \times \cdots \times \tau_{R}$. Now $\tau_{0}$ and $\tau_{R}$ are arbitrary (e.g., $\tau_{0}=\pi^{t}, \tau_{R}=\pi^{L-t}$ for some probability measure on $\left.\Omega_{1}\right)$. If $1 \leqslant j \leqslant R-1, \tau_{j}$ is defined as the conditional distribution of $\left(X_{0}^{(1)}, \ldots, X_{L-1}^{(1)}\right)$ given

$$
\left(X_{0}^{(2)}, \ldots, X_{L-1}^{(2)}\right)=\left(\beta_{r}, \beta_{r+1}, \ldots, \beta_{r+L-1}\right), \quad m=k M+t+(j-1) L .
$$


If $L-1 \leqslant i \leqslant M-L-q$, then because $F$ depends only on the first $q$ coordinates (in both factors $\left.X_{1}, X_{2}\right)$, the integral $\left(F_{i}, \lambda\right)$ is effectively an integral $\left(F_{i}, \eta_{k}\right)$, where $k=[i / M]$. Secondly, $\eta_{k}=L^{-1} \sum_{t=0}^{L-1} \sigma_{t}^{k}$, and for all but at most $q$ values of $t\left(F_{i}, \sigma_{t}^{k}\right)$ equals one of the summands in (1.8.8). This gives some idea as to why (1.8.8) is true.

1.8.10. Question. To what extent does Kamae's theorem generalize? Is it true when $\mathfrak{X}_{1}$ satisfies specification and $\mathfrak{X}_{2}$ is arbitrary?

1.9. Normal sequences and collectives. Von Mises [94] defined a collective to be a sequence $x=\left\{x_{n}\right\}$ of zeros and ones in which 1 occurs with relative frequency (say) $\frac{1}{2}$, and such that if $\tau=\{\tau(1)<\tau(2)<\cdots\}$ is any "selection rule", 1 also occurs in $x \circ \tau=\left\{x_{\tau(n)}\right\}$ with frequency $\frac{1}{2}$. Such sequences cannot exist, but if the notion is suitably modified, they do. The definitions by Weiss and Kamae below are similar in spirit to one by Wald (Ville [152]).

Let $\Omega$ be a compact metric space, and let $Q X=(T, X)$ be the left shift on $X=\Omega^{N}$. If $\lambda$ is a Borel probability measure on $\Omega$, a sequence $x \in X$ is $\lambda$ normal if it is generic for $\mu=\lambda^{N}(=\lambda \times \lambda \times \cdots)$. If $\tau=\{\tau(1)<\tau(2)$ $\langle\cdots\}$ is a selection rule, a $\lambda$-normal sequence is a $\tau$-collective if $x \circ \tau$ is again $\lambda$-normal [68]. Of course, if $\tau$ is fixed, $\mu$-almost all $x$ are $\tau$ collectives.

There is a natural one-to-one correspondence between selection rules $\tau$ and points $\xi=\xi_{\tau} \in\{0,1\}^{N}$ which have infinitely many ones. We say $\tau$ is deterministic if for every $\nu \in V\left(\xi_{\tau}\right)$ (relative to the left shift) the partition $P=\left\{A_{0}, A_{1}\right\}, A_{j}=\{\xi \mid \xi(0)=j\}$, is measurable $(\nu)$ with respect to $P_{1}^{\infty}$ $=\bigvee_{j=1}^{\infty} T^{-j} P$. For example, if $\alpha>1$ the selection rule $\tau(n)=[n \alpha]$ may be seen to be deterministic.

Next, $\tau$ is said to be admissible if $\lim _{\sup _{n \rightarrow \infty}}(\tau(n) / n)<\infty$.

1.9.1. TheOREM (Weiss [156], KAMAe [68]). Let $\tau$ be a selection rule which is both admissible and deterministic. Then for any $\Omega, \lambda$ every $\lambda$-normal sequence is a $\tau$ collective.

It was conjectured by Weiss that the converse to the theorem above would be true. The remarkable fact that it is true was proved by Kamae.

1.9.2. Theorem (KAmAe [68]). Let $\tau$ be a selection rule, and let $\lambda$ be a nontrivial probability measure on $\Omega$. If every $\lambda$-normal sequence is a $\tau$-collective, then $\tau$ is deterministic.

If one thinks of a selection rule as a "strategy", then the rules above are very special, being the same for any sequence encountered. Weiss and Kamae [69] define a set $S \subseteq\{0,1\}^{*}=\cup_{n=1}^{\infty}\{0,1\}^{n}$ to be a selection rule, the "rule" being that $x_{i}$ is selected if and only if $\left(x_{1}, x_{2}, \ldots, x_{i-1}\right) \in S$. See [69] for a number of interesting examples and questions.

1.10. Disjointness in ergodic theory. In this section $x, \mathcal{Q}, \ldots$, unless otherwise specified, denote processes, i.e., endomorphisms $T$ of probability spaces $\left(X, \Re_{X}, \mu_{X}\right),\left(Y, \Re_{Y}, \mu_{Y}\right) \cdots$. The notions of product, homomorphism, factor, etc., carry over naturally to processes. For example, of $\rightarrow^{\pi} \mathscr{O}$ is a homomorphism if $\pi: X \rightarrow Y$ is measurable, $\pi \mu_{X}=\mu_{Y}$, and $\pi T=T \pi$ a.e.

Processes $x_{1}$ and $x_{2}$ are disjoint if whenever $x^{\pi_{j}} \mathfrak{x}_{j}, j=1,2$, are homomorphisms, $\pi_{1} \times \pi_{2}$ is also; that is, $\pi_{1} \times \pi_{2} \mu_{X}=\mu_{X_{1}} \times \mu_{X_{2}}$ (Furstenberg 
[48]). Disjointness may be viewed as "absolute independence" because it is equivalent to require that $\pi_{1}^{-1} \Re_{X_{1}}$ and $\pi_{2}^{-1} \mathscr{G}_{X_{2}}$ be independent sub- $\sigma$-algebras of $\mathscr{B}_{X}$. Disjoint processes are relatively prime (a fact which is not trivial in the case of processes), and if two processes are disjoint, at least one is ergodic (compare with the corresponding fact for flows).

It is necessary now to recall the notion of entropy in ergodic theory. Define $\eta(t)=-t \log t, 0 \leqslant t \leqslant 1$, with $0 \log 0=0$. If $P$ is a finite partition of $X$ (all partitions are measurable), define the entropy of $P$ by $H(P)$ $=\sum_{B \in P} \eta(\mu(B))$. Then, letting $P_{0}^{n}$ be the join $\vee_{j=0}^{n} T^{-j} P$, the entropy of $T$ relative to $P$ is defined to be $h(T, P)=\lim _{n \rightarrow \infty}\left(H\left(P_{0}^{n}\right) /(n+1)\right)$, the limit existing. Always, $0 \leqslant h(T, P) \leqslant H(P)$, and $h(T, P)=0$ if and only if $P$ is measurable $P_{1}^{\infty}=\bigvee_{j=1}^{\infty} T^{-j} P$ (that is, $P$ is deterministic relative to $T$ ). Finally, $h(T)$, the entropy of $T$ (Kolmogorov-Sinai invariant) is $\sup _{P} h(T, P)$. If $P_{0}^{\infty}=\mathscr{B}_{X}$, or if $T$ is an automorphism and $P_{-\infty}^{\infty}=\mathscr{B}_{X}$, then $h(T)=h(T, P)$ (Kolmogorov-Sinai theorem). For a discussion of the elementary properties of entropy, see Walters [155].

When more than one invariant measure is considered on the same space, dependence of entropy upon the measure will be expressed by $h_{\mu}(T, P), h_{\mu}(T)$, etc.

A process $\mathfrak{X}$ is deterministic if $h(T)=0$. It is a Pinsker process (process with completely positive entropy) if $h(T, P)>0$ whenever $P$ is a nontrivial partition. As noted in [48], "Pinsker's Lemma" is equivalent to the following:

1.10.1. TheORem ("PinsKer's Lemma"). If $x_{1}$ and $x_{2}$ are, respectively, a Pinsker process and a deterministic process, then $\mathfrak{X}_{1}$ and $\mathfrak{X}_{2}$ are disjoint.

Pinsker processes include Bernoulli shifts (the left shift relative to $\mu=\lambda^{N}$ or $\left.\lambda^{\mathbf{Z}}\right)$, ergodic automorphisms of tori, and $x \rightarrow r x(\bmod 1)$, while deterministic processes include affine transformations of the torus $\left(x \rightarrow x_{0}+\sigma x, \sigma\right.$ an automorphism, $x_{0}$ fixed) such that the integer matrix associated to $\sigma$ is unipotent. See [135] for a proof of Pinsker's Lemma.

1.10.2. Definition. Let $\mathfrak{X}=(T, X)$ be a cascade. A point $x \in X$ is deterministic (relative to $T$ ) if $h_{\mu}(T)=0$ for all $\mu \in V(x)$.

In the special case that $T$ is the left shift on $\Omega_{r}^{N}, \Omega_{r}=\{0,1, \ldots, r-1\}$, Rauzy [111] has given an interesting and useful characterization of deterministic sequences. For each $s>0$ denote by $E_{s}$ the set of maps $\Omega_{r}^{s} \rightarrow \Omega_{r}$. Given $x=\left(x_{0}, x_{1}, \ldots\right) \in X$ and $M, s>0$, define

$$
\beta_{s}(x, M)=\min _{\varphi \in E_{s}} \frac{1}{M} \sum_{n<M} \min \left(1,\left|x_{n}-\varphi\left(x_{n+1}, \ldots, x_{n+s}\right)\right| .\right.
$$

If $x$ is periodic with period $\left\langle s, \beta_{s}(x, M)=0\right.$. The lim sup and lim inf, as $M \rightarrow \infty$, of (1.10.3) are denoted $\beta_{s}^{\prime}(x)$ and $\beta_{s}^{\prime \prime}(x)$. These quantities are nonincreasing in $s$, and their limits, as $s \rightarrow \infty$, are denoted $\beta^{\prime}(x)$ and $\beta^{\prime \prime}(x)$ ("lower noise" and "upper noise" of $x$ ). Always,

$$
0 \leqslant \beta^{\prime}(x) \leqslant \beta^{\prime \prime}(x) \leqslant(r-1) / r .
$$

1.10.4. Proposition (RAUZy [111]) $x \in X=\Omega_{r}^{N}$ is deterministic if and only if $\beta^{\prime \prime}(x)=0$. 
For example, suppose $x$ is deterministic, and fix $\varepsilon>0$. For each $\mu \in V(x)$ there exist, because $h_{\mu}(T)=0, s$ and $\varphi \in E_{s}$ such that if $f_{\varphi}(y)=$ $\varphi\left(y_{0}, y_{1}, \ldots, y_{s-1}\right)$, then $\left\|X_{0}-T f_{\varphi}\right\|_{1}<\varepsilon$, where $X_{0}$ is the 0 th coordinate, and $\|\cdot\|_{1}$ is with respect to $\mu$. By the weak-* compactness of $V(x)$, there is a finite set $\left\{\left(s_{j}, \varphi_{j} \in E_{s_{j}}\right)\right\}$ such that $\sup _{\mu \in V(x)} \min _{j}\left\|X_{0}-T f_{\varphi_{j}}\right\|_{1}<\varepsilon$. Now let $s$ $=\max _{j} s_{j}$, and choose $S \subseteq N$ so that (a) $\beta^{\prime \prime}(x)=\lim _{n \rightarrow \infty ; n \in S} \beta_{s}^{\prime \prime}(x, n)$, and (b) $S \in \Gamma_{x}$ ( $\mu_{x}^{S}$ exists). If $\left(s_{j}, \varphi_{j}\right)$ is associated to $\mu_{x}^{S}$ above, then as $s_{j} \leqslant s$, there is $\varphi \in E_{s}$ with $f_{\varphi_{j}}=f_{\varphi}$. Now

$$
\varepsilon>\left\|X_{0}-T f_{\varphi}\right\|_{1}=\lim _{\substack{n \rightarrow \infty \\ n \in S}} \frac{1}{n} \sum_{j=1}^{n}\left|x_{j}-\varphi\left(x_{j+1}, \ldots, x_{j+s}\right)\right| \geqslant \beta_{s}^{\prime \prime}(x) .
$$

Thus, $\beta^{\prime \prime}(x) \leqslant \beta_{s}^{\prime \prime}(x)<\varepsilon$, and letting $\varepsilon \rightarrow 0, \beta^{\prime \prime}(x)=0$. The converse has a similar proof.

EXAMPle (RAUZY [112]). Let $r=2$ and define $x_{0}=0, x_{n}=|\mu(n)|, n \geqslant 1$, where $\mu(\cdot)$ is the Möbius function. If $p$ is prime, define $\nu_{p}(n)=1$ if $p^{2} \nmid n,=0$ if $p^{2} \mid n$. Then $x_{n}=\prod_{p} v_{p}(n)$. If $l>0$, define $x_{n}^{(l)}=\prod_{p \leqslant l} v_{p}(n)$. Then $x^{(l)}$ is periodic of period $s(l)=\prod_{p \leqslant l} p^{2}$, and $x_{n}^{(l)} \neq x_{n}$ only if $q^{2} \mid n$ for some $q>l$. Define $\varphi \in E_{s(l)}$, first as $\varphi\left(y_{n}\right)=x_{n}^{(l)}$, where $y_{n}=\left(x_{n+1}^{(l)}, \ldots, x_{n+s(l)}^{(l)}\right), 0 \leqslant n$ $<s(l)$. It is elementary that the points $y_{n}$ are distinct, and therefore for any $m$, if $z_{m}=\left(x_{m+1}, \ldots, x_{m+s(l)}\right)$, there is a unique $n$ such that $z_{m} y_{n}=y_{n}$ (multiply coordinate-wise). Define $\varphi\left(z_{m}\right)=\varphi\left(y_{n}\right)$, and then extend $\varphi$ arbitrarily to $E_{s}$. Now $\varphi\left(z_{m}\right)=x_{m}$ if and only if $x_{m}^{(l)}=x_{m}$. As the set $\left\{m \mid x_{m}^{(l)} \neq x_{m}\right\}$ has upper density at most $\sum_{q>l} 1 / q^{2}<1 / l$, we have $\beta_{s(l)}^{\prime \prime}(x) \leqslant 1 / l$. Letting $l \rightarrow \infty, \beta^{\prime \prime}(x)$ $=0$, and $x$ is deterministic.

It follows from the Weiss-Kamae theorem and the above that if $a_{1} a_{2} a_{3} \ldots$ is a normal number to some base, then so is the number $a_{1} a_{2} a_{3} a_{5} a_{7} a_{10} a_{11} a_{13} \ldots$ !

1.11. The theorems of Weiss, Kamae, and Rauzy. I shall first sketch a proof of Weiss' Theorem 1.9.1, following Kamae. It is sufficient to prove that if $\tau$ is an admissible and deterministic selection rule, and if $x$ is a $\lambda$-normal sequence, then for all $s>0$ and $f \in C\left(\Omega^{s}\right)$,

$$
\lim _{M \rightarrow \infty} \frac{1}{M} \sum_{n=1}^{M} f\left(x_{\tau(n)}, \ldots, x_{\tau(n+s-1)}\right)=\int_{\Omega^{s}} f \lambda^{s} .
$$

(All sequences will be numbered beginning with 1.) The case $s=2$ is typical. $\mathcal{X}$ denotes the left shift on $\Omega^{N} \times\{0,1\}^{N}$. Let $\Delta \subseteq\{0,1\}^{N}$ be the sequences with infinitely many 1 's, and set $\Delta^{\prime}=\Delta \cup\{0\}$. One verifies easily that $\nu\left(\Omega^{N} \times \Delta^{\prime}\right)=1$ for all $\nu \in \mathscr{P}_{I}(\mathfrak{X})$. Therefore, if we associate to $\xi \in \Delta$ the rule $\tau_{\xi}(n)=$ index of $n$th occurrence of 1 in $\xi$, the function on $\Omega^{N} \times \Delta^{\prime}$,

$$
g(y, \xi)= \begin{cases}f\left(y_{1}, y_{\tau_{\xi}}(2),\right. & \xi(1)=1 \\ 0, & \xi(1)=0\end{cases}
$$

being continuous is continuous a.e. $\nu$. In particular, if $\nu_{n} \rightarrow \nu$ weak-*, then $\left(g, v_{n}\right) \rightarrow(g, v)$.

Let $\Delta_{\infty}=\{\xi \in \Delta \mid \xi(1)=1\}$, and let $\Delta_{m}, m \geqslant 2$, be $\left\{\xi \in \Delta_{\infty} \mid \tau_{\xi}(2)=m\right\}$. Then $\Delta_{\infty}=\cup_{m=2}^{\infty} \Delta_{m}$. If $\nu \in V\left(\left(x, \xi_{\tau}\right)\right)$, then because $x$ is $\lambda$-normal and $\tau$ is 
deterministic, $\left.\nu\right|_{\Omega^{N}}=\lambda^{N}$ and $\left.\nu\right|_{\{0,1\}^{N}}=\mu$ satisfies $h_{\mu}(T)=0$. By Pinsker's Lemma, $\nu=\lambda^{N} \times \mu$, and by (1.11.2),

$$
(g, \nu)=\sum_{m=2}^{\infty} \mu\left(\Delta_{m}\right)\left(f, \lambda^{2}\right)=\mu\left(\Delta_{\infty}\right)\left(f, \lambda^{2}\right) .
$$

Now let $S \subseteq N$ be any sequence such that as $M \rightarrow \infty, M \in S$, the left side of (1.11.1) converges to some number $\alpha$ and the measures $\mu_{\left(x, \xi_{T}\right)}^{\tau(M)}$ converge to some $\nu=\lambda^{N} \times \mu$. It will suffice to prove $\alpha=\left(f, \lambda^{2}\right)$. Note that $\lim _{M \rightarrow \infty ; M \in S}(M / \tau(M))=\mu\left(\Delta_{\infty}\right)>0$ because $\tau$ is admissible. Now write

$$
\begin{aligned}
\frac{1}{M} \sum_{n=1}^{M} f\left(x_{\tau(n)}, x_{\tau(n+1)}\right) & =\frac{1}{M} \sum_{j=0}^{\tau(M)-1} g\left(T^{j}\left(x, \xi_{\tau}\right)\right) \\
& =\frac{\tau(M)}{M} \frac{M}{\tau(M)} \sum_{j=0}^{\tau(M)-1} g\left(T^{j}\left(x, \xi_{\tau}\right)\right) .
\end{aligned}
$$

By (1.11.3) and the preceding remark, the terms on the right approach $\left(\mu\left(\Delta_{\infty}\right)\right)^{-1} \cdot \mu\left(\Delta_{\infty}\right) \cdot\left(f, \lambda^{2}\right)=\left(f, \lambda^{2}\right)$ as $M \rightarrow \infty, M \in S$. Thus, $\alpha=\left(f, \lambda^{2}\right)$, and (1.11.1) holds (for $s=2$ ).

Kamae's theorem, Theorem 1.9.2, is considerably deeper, relying both on the machinery developed in $\$ 8$ as well as on the following generalization of a theorem of Furstenberg, in which, as Kamae notes, there was a gap. Let $\mathfrak{X}_{1}=\left(T, \Omega^{N}\right), \mathscr{X}_{2}=\left(T,\{0,1\}^{N}\right)$ be the shifts.

1.11.5. TheOREM (Furstenberg [48], KAMAe [68]). Let $\mu \in \mathscr{P}_{I}\left(\mathfrak{X}_{2}\right)$ be such that $h_{\mu}(T)>0$. If $\lambda$ is nontrivial, there exists $\nu \in \mathscr{P}_{I}(\mathcal{X})$ such that $\left.\nu\right|_{\Omega^{N}}=\lambda^{N}$ and $\left.\nu\right|_{\{0,1\}^{N}}=\mu$, but the first coordinate functions $X_{1}(x, y)=x_{1}$ and $Y_{1}(x, y)$ $=y_{1}$ are not independent. (In particular, $\nu \neq \lambda^{N} \times \mu$.)

Suppose now that $\tau$ is a selection rule and that every $\lambda$-normal sequence is a $\tau$ collective. We shall derive a contradiction from the assumption that some $\mu \in V\left(\xi_{\tau}\right)$ has $h_{\mu}(T)>0$. Fixing such a $\mu$, choose $\nu \in \mathscr{P}_{I}(\mathcal{X})$ as in the theorem. If $S \in \Gamma_{\xi_{\tau}}$ is such that $\mu_{\xi_{T}}^{S}=\mu$, then by Theorem 1.8.6 there exists a $\lambda$-normal sequence $x$ such that $\left.\mu_{\left(x, \xi_{\tau}\right.}^{S_{\tau} \tau}\right)=\nu$. Because $X_{1}, Y_{1}$ are not independent (and $Y_{1}$ is 0,1 valued), there exists $f \in C(\Omega)$ such that $\left(Y_{1} f \circ X_{1}, v\right)$ $\neq \mu\left(\Delta_{\infty}\right)(f, \lambda)$. Now if $L_{n}=\operatorname{Card}\{m \mid \tau(m) \leqslant n\}$, then $L_{n} / n$ tends to $\mu\left(\Delta_{\infty}\right)$ as $n \rightarrow \infty, n \in S$. Coupling this with the assumed normality of $x \circ \tau$, we have

$$
\begin{aligned}
\left(Y_{1} f \circ X_{1}, \nu\right) & =\lim _{\substack{n \rightarrow \infty \\
n \in S}} \frac{1}{n} \sum_{j=1}^{n} Y_{1}\left(T_{\xi_{\tau}}^{j}\right) f\left(T_{x}^{j}\right) \\
& =\lim _{\substack{n \rightarrow \infty \\
n \in S}} \frac{L_{n}}{n} \frac{1}{L_{n}} \sum_{m=1}^{1} f\left(x_{\tau(m)}\right)=\mu\left(\Delta_{\infty}\right)(f, \lambda)
\end{aligned}
$$

which contradicts the choice of $f$.

Rauzy has used the work of Kamae to give a remarkable solution to a problem about normal numbers posed by Mendes-France [93]. Define $B(r)$ to be the set of $r$ normal numbers and $B^{\perp}(r)$ to be the set of $x \in R$ such that $x+B(r) \subseteq B(r)$. It can be shown that $B^{\perp}(r)$ is a linear subspace of $\mathbf{R}$ over $\mathbf{Q}$, 
and since it clearly contains 1 , it contains $\mathbf{Q}$. If $x$ is irrational, associate to it the $r$-ary expansion of its fractional part, $x=. b_{1} b_{2} \cdots(\bmod 1)$, and let $b=\left(b_{1}, b_{2}, \ldots\right) \in \Omega_{r}^{N}$. Call $x$ deterministic to the base $r$ if $b$ is deterministic for the shift, and let $D(r)$ be the set of $r$-deterministic numbers $(\cup \mathbf{Q})$. The theorem of Rauzy is

\subsubsection{Theorem (G. RAUZY [111]). $B^{\perp}(r)=D(r)$.}

In a later paper [110], Rauzy characterizes those sequences $\left\{x_{n}\right\}$ of real numbers with the property that whenever $y=\left\{y_{n}\right\}$ is uniformly distributed modulo $1,\left\{x_{n}+y_{n}\right\}$ is also uniformly distributed. The condition is rather natural, but complicated to state, and the reader is referred to [110] for further details.

1.12. Regularity and irregularity of uniform distribution. Let $K=\mathbf{R} / \mathbf{Z}$, and let $\theta \in K$ be irrational. If $I \subseteq K$ is an interval, define $S_{n}(\theta, I)$ $=\sum_{j=0}^{n-1} \chi_{I}\left(j^{\prime} \theta\right)$. By the Kronecker-Weyl theorem,

$$
\lim _{n \rightarrow \infty} \frac{S_{n}(\theta, I-x)}{n}=|I|
$$

holds uniformly in $x$, where $|I|$ is the length of $I$. Below we shall consider more delicate questions about the sequence $\left\{S_{n}\right\}$.

Consider, for example, the sequence $S_{n} \alpha-n \beta$ for any fixed $\alpha, \beta \in \mathbf{R}$. If $f$ is defined on $K$ by

$$
f=e^{2 \pi i(\alpha-\beta)} \chi_{I}+e^{-2 \pi i \beta} \chi_{I^{c}}
$$

and if $f^{(n)}(x)=f(x) f(x+\theta) \cdots f(x+(n-1) \theta)$, then

$$
f^{(n)}(x)=\exp 2 \pi i\left(S_{n} \alpha-n \beta\right), \quad S_{n}=S_{n}(\theta, I-x) .
$$

Therefore by Lemma 1.2.5, applied to $P=\left\{I, I^{c}\right\}$ and $T x=x+\theta$, if for some $x$,

$$
\lim _{M \rightarrow \infty} \frac{1}{M} \sum_{n=1}^{M} \exp \left(2 \pi i\left(S_{n} \alpha-n \beta\right)\right)=0
$$

fails to hold, the equation $h(x)=f(x) h(x+\theta)$ has a measurable solution which is not essentially 0 . Since $|h(x+\theta)|=|h(x)|,|h|$ is constant, and we may take the constant to be 1 . Therefore

$$
h(x) \bar{h}(x+\theta)=f(x), \text { and } h(x) \bar{h}(x+n \theta)=f^{(n)}(x), \quad n>0 .
$$

Setting $\alpha_{n}=\int_{K} f^{(n)}, \alpha_{n} \rightarrow 1$ as $n \theta \rightarrow 0$ in $K$. In particular,

$$
\lim _{n \rightarrow 0}\left|\alpha_{n}\right|=1 \text {. }
$$

Since (1.12.3) does not depend upon $\beta$, we define $\Gamma_{\alpha}(\theta)$ to be the set of $t$ such that if $|I|=t$, then (1.12.3) holds. (We regard $t$ as an element of $K$.)

Let $\|\cdot\|$ be the usual metric on $K$ (distance to nearest integer), and let $\left\{p_{n} / q_{n}\right\}$ be the sequence of convergents to $\theta[80]$. Define $\Gamma^{0}(\theta)$ to be the set of $t \in K$ such that $t=\sum_{n=1}^{\infty} b_{n} q_{n} \theta$, where convergence is understood in terms of $\|\cdot\|$, 
and such that $\lim _{n \rightarrow \infty} b_{n} q_{n}\left\|q_{n} \theta\right\|=0$. $\Gamma^{0}(\theta) \supseteq \mathbf{Z} \theta$, and if $\theta$ has bounded partial quotients $\Gamma^{0}(\theta)=\mathbf{Z} \theta$; otherwise, $\Gamma^{0}(\theta)$ is uncountable but of measure 0 . Also, $\Gamma^{0}(\theta)$ contains no rational, other than 0 . If $t \in \Gamma^{0}(\theta)$, the $b_{n}$ 's are determined for large $n$ [149], and therefore it makes sense to define $\Gamma_{\alpha}^{0}(\theta)$ $=\left\{t \in \Gamma^{0}(\theta) \mid \lim _{n \rightarrow \infty} b_{n} \alpha=0(\bmod 1)\right\}$.

\subsubsection{THEOREM [149], [151]. $\Gamma_{\alpha}(\theta) \subseteq \Gamma_{\alpha}^{0}(\theta)$.}

The theorem was proved for $\alpha=\frac{1}{2}$ in [149] and a proof was sketched for general $\alpha$ in [151]. A complete proof will appear in a forthcoming Rice Ph.D. thesis.

The implications of Theorem 1.12.4 for (1.12.3) are as follows:

1.12.5. THEOREM. With notations as above we have (a) if $\theta$ has bounded partial quotients, then (1.12.2) holds for all $|I| \in \mathbf{Z}$ and $(\alpha, \beta) \notin \mathbf{Z} \times \mathbf{Z}$; (b) if $\theta$ is irrational, then for almost all $|I|,(1.12 .2)$ holds for all $(\alpha, \beta) \notin \mathbf{Z} \times \mathbf{Z}$; (c) for any irrational $\theta$ and $|I|$ it is true for almost all $\alpha$ that (1.12.2) holds for all $\beta$; and (d) if $\theta$ is irrational and $|I|$ rational, (1.12.2) holds for all $(\alpha, \beta) \notin \mathbf{Z} \times \mathbf{Z}$.

The point of (c) is that if $|I| \notin \mathbf{Z} \theta$ but $|I| \in \Gamma^{0}(\theta)$, the set of $\alpha$ with $|I| \in \Gamma_{\alpha}^{0}(\theta)$ has measure 0 by the Riemann-Lebesgue lemma. A special case of $(\mathrm{d})$ is proved by Rauzy [113].

Now define

$$
\Gamma_{\alpha}^{1}(\theta)=\left\{t \in \Gamma_{\alpha}^{0}(\theta)\left|\sum_{n=1}^{\infty}\right| b_{n} \mid q_{n}\left\|q_{n} \theta\right\|<\infty\right\}
$$

We have

$$
\text { 1.12.6. THEOREM [149]. } \Gamma_{1 / 2}^{1}(2 \theta) \subseteq \Gamma_{1 / 2}(\theta) \text {. }
$$

In [149] it is proved that if $t \in \Gamma_{1 / 2}(\theta)(t \notin \mathbf{Z} \theta)$ and if $\alpha=\frac{1}{2}, \beta=0$, there exists a choice of $I=[0, t)$ or $[t, 1)$ and uncountably many values of $x$ such that the limit (1.12.2) does not even exist. Since $\Gamma_{1 / 2}^{1}(2 \theta)$ is uncountable when $\theta$ has unbounded partial quotients, Theorems $1.12 .5-1.12 .6$ may be combined to imply

1.12.7. THEOREM. The limit (1.12.2) exists for all $\alpha, \beta$, and $|I|$ if and only if $\theta$ has bounded partial quotients (when $|\boldsymbol{I}| \notin \mathbf{Z} \theta,(\alpha, \beta) \notin \mathbf{Z} \times \mathbf{Z}$, it is 0 ).

Returning to Theorem 1.12.5(c), note that for almost all $\alpha$ the statement is true for all $n \alpha, n= \pm 1, \pm 2, \ldots$. This plus Weyl's criterion implies that if $|I| \notin \mathbf{Z} \theta$, then for almost all $\alpha$ the sequence $\left\{S_{n} \alpha-n \beta\right\}$ is uniformly distributed modulo 1 for every $\beta$. In particular, if we set $\beta=|I| \alpha$, it follows that

1.12.8. Theorem. If $\theta$ is irrational and $|I| \notin \mathbf{Z} \theta$, then for almost all $\alpha$ the sequence $\left(S_{n}-n|I|\right) \alpha$ is uniformly distributed modulo 1.

The theorem implies that if $\theta$ is irrational and $|I| \notin \mathbf{Z} \theta$, the sequence $\left(S_{n}-n|I|\right)$ is not bounded for $n \geqslant 1$. For if it were there could not exist $\alpha$ 's arbitrarily close to 0 for which $\left(S_{n}-n|I|\right) \alpha$ is uniformly distributed modulo 1. Thus we retrieve a theorem due to Kesten: 
1.12.9. THEOREM (KESTEN [76]). Let $\theta$ be irrational and $I \subseteq K$ an interval. If there exists $x \in K$ such that the sequence $\left\{S_{n}(\theta, I-x)-n|I|\right\}$ is bounded for $n \geqslant 1$, then $|I| \in \mathbf{Z} \theta$ (and conversely [62], [99]).

There are some interesting and perhaps difficult problems to consider in connection with both our work and Kesten's Theorem. Let $I \subseteq K$, and let $E$ be the closed subgroup of $\mathbf{R}$ (not $K$ ) generated by $|I|$ and $1-|I|$. Of course, $E=\mathbf{R}$ if $|I|$ is irrational. Now set up the space $X=K \times E$, and define $T$ on $X$ by $T(x, y)=\left(x+\theta, y+\chi_{I}(x)-|I|\right)$. Notice that the second coordinate of $T^{n}(x, y), n>0$, is $y+S_{n}-n|I|$. Thus, by Kesten's Theorem, if $|I| \notin \mathbf{Z} \theta$, there are no bounded orbits under $T$.

1.12.10. Problem. If $|I| \notin \mathbf{Z} \theta$, is $(T, X)$ topologically transitive?

1.12.11. Problem. If $|I| \notin \Gamma^{0}(\theta)$, is $T$ ergodic relative to Haar measure on $X$ ?

1.12.12. Problem. When $T$ is ergodic relative to Haar measure, can there exist a locally finite invariant measure which is not a multiple of Haar measure?

In connection with Problem 1.12.10, Nelson Markley has shown there necessarily exist points with semibounded orbits, and therefore $(T, X)$ cannot be minimal (oral communication.) In connection with the second problem a special case is contained in K. Schmidt $\left([121], \theta=(\sqrt{5}-1) / 4,|I|=\frac{1}{2}\right)$ and Conze and Keane ([24], $\theta$ irrational, $\left.|I|=\frac{1}{2}\right)$. As for the third, the locally finite assumption is necessary, not only to prevent trivial counterexamples, but also because there exist infinite nonatomic measures on $K$ which are invariant and ergodic under rotation by $\theta$ [122], [123].

For later reference I shall state here another theorem in the same spirit as Kesten's.

1.12.13. Theorem (Furstenberg, Keynes, Shapiro [51]). Assume 2|I| $\notin \mathbf{Z} \theta$. If $x-x^{\prime} \notin \mathbf{Z} \theta$, the sequence $S_{n}(\theta, I-x)-S_{n}\left(\theta, I-x^{\prime}\right)$ is unbounded.

1.13. The construction of prime flows. I shall begin with some "soft" counterparts to certain results from the previous section. The axiomatics are taken essentially from [149].

Let $(X, \theta)$ be a compact abelian group and an element which generates a dense subgroup. Let $\Lambda$ be a compact abelian group, and $f: F^{c} \rightarrow \Lambda$ a continuous map defined on the complement of a closed, nowhere dense set $F \subseteq X$. I shall call $(f, \theta)$ admissible if (a) at each point of $F, f$ has at most two cluster values, while at some point it has two whose difference generates a dense subgroup of $\Lambda$, (b) $f$ has no nonzero period, and (c) $(F-F) \cap \mathbf{Z} \theta$ $=\{0\}$.

Let $A=\cup_{n \in \mathbf{Z}}(F+n \theta)$, and define $m_{x} \in \Lambda^{\mathbf{Z}}, x \in A^{c}$, by $m_{x}(n)=$ $f(x+n \theta)$. Since $m_{x+\theta}=\sigma m_{x}$, where $\sigma$ is the shift, the closure $M$ of $\left\{m_{x} \mid x \in A^{c}\right\}$ is $\sigma$-invariant.

1.13.1. Proposition [149], [108], [51]. If $(f, \theta)$ is admissible, then 9 . is a minimal flow. The map $\pi m_{x}=x, x \in A^{c}$, extends to be continuous on $M$. Moreover, $\pi^{-1} x=\left\{m_{x}\right\}, x \in A^{c}$, and, in general, if $\pi m_{1}=\pi m_{2}$, then $m_{1}(n)$ $\neq m_{2}(n)$ for at most one value of $n$. 
Next let $N=M \times \Lambda$, and define $T(m, \lambda)=(\sigma m, m(0) \lambda)$ on $N$.

1.13.2. Proposition [149]. If $(f, \theta)$ is admissible, then $\Re=(T, N)$ is a minimal flow.

In [149] the proposition is proved for $\Lambda$ the group of order 2 , but the same proof works in the general case. The idea is that if $N^{\prime}=\theta\left(\left(m_{0}, 1\right)\right), m_{0} \in M$ fixed, where $\theta(\cdot)$ denotes orbit closure, then, for each $m,\left\{\lambda \mid(m, \lambda) \in N^{\prime}\right\}$ is a coset of a closed subgroup $\Lambda_{0}\left(=\Lambda m_{0}\right)$. If $h(m)$ denotes the coset, then $h$ is continuous and $h(\sigma m)=m(0) h(m)$. The set $E$ of $x$ such that $h$ has two values on $\pi^{-1} x$ is closed, and (c) may be seen to imply it is dense, if nonempty. Thus, $E=\varnothing$, meaning in particular, that for each $x,\left\{m(0) \mid m \in \pi^{-1} x\right\}$ is contained in one coset of $\Lambda_{0}$. By (a) then, $\Lambda_{0}=\Lambda$, and $\Re$ is minimal.

EXAmple. $X=K, \theta \in K$ irrational, $I \subseteq K$ an interval with $|I| \notin \mathbf{Z} \theta$. Let $\alpha, \beta \in K$ with $\alpha \neq 0$, and assume $\beta \in \Lambda$, where $\Lambda$ is the closed subgroup generated by $\alpha$. Define $f$ on $I^{0}$ as $f=\alpha-\beta$ and on $\left(I^{c}\right)^{0}$ as $f=-\beta$. Then $(f, \theta)$ is admissible. (Note that $F=\partial I$.) Now if $x \in A^{c}$ and $n>0$, a straightforward calculation reveals that $T^{n}\left(m_{x}, 0\right)=\left(m_{x+n_{\theta}}, S_{n}^{x} \alpha-n \beta\right)$, where $S_{n}^{x}=S_{n}(\theta, I-x)$. The following theorem is a consequence of the minimality of $\Re$ above:

1.13.3. THEOREM. With notations and assumptions as above, if $x, y \in K$ and $\varepsilon>0$ are given, the set

$$
\left\{S_{n}^{x} \alpha-n \beta \mid\|x+n \theta-y\|<\cdot \varepsilon\right\}
$$

is dense in $\Lambda$. In particular, if $\alpha$ is irrational, then (1.13.4) is dense in $K$.

Taking $\beta=\alpha|I|$ in the theorem leads to another proof of Kesten's theorem. A different $f$ can be used to prove Theorem 1.12.10. Taking $\alpha=1 / q, \beta$ $=1-1 / q$ leads to

1.13.5. Corollary. With notations as above the set $\left\{n \theta|q|\left(n+S_{n}^{x}\right)\right\}$ is dense in $K$. (Here $q \mid s$ means " $q$ divides $s "$.)

I call Theorem 1.13.3 a "soft" counterpart to the corresponding results of $\$ 1.12$ because first of all it depends only upon $\theta$ 's being irrational and $\alpha \neq 0$, and second it is a minimality theorem, whereas the results of $\$ 1.12$ correspond to unique ergodicity (of $\mathcal{x}$ ) theorems (see [49], [149]). When $\Lambda$ is nonabelian, very few "soft" results are known (see [142]).

Furstenberg, Keynes, and Shapiro [51] base their proof of Theorem 1.12.13 (and Kesten's theorem) on a principle of Gottschalk and Hedlund [58]: If x $=(T, X)$ is minimal, and if $h \in C(X), x_{0} \in X$ are such that $\sum_{j=0}^{n} h\left(T^{j} x_{0}\right)$ is bounded for $n>0$, then there exists $g \in C(X)$ such that $g(T x)-g(x)$ $=h(x)$. (The converse is trivial.) From this they infer

1.13.6. Proposition ([51]; SEE ALSO [107]). Let $I \subseteq X, x_{0} \in X, \delta \in \mathbf{R}$ be such that $\sum_{j=0}^{n}\left(\chi_{I}\left(T^{n} x_{0}\right)-\delta\right)$ is bounded for $n>0$. Let $M$ be the orbit closure of $m, m(n)=\chi_{I}\left(T^{n} x_{0}\right)$, in $\left(\sigma,\{0,1\}^{\mathbf{Z}}\right)$, and assume $(\sigma, M)$ is minimal. Then there exists a nonzero $g \in C(M)$ such that $g(\sigma m)=\exp (2 \pi i \delta) g(m)$.

Kesten's theorem is proved, for example, by taking $\delta=|I|$ and noting that any eigenvalue for $(\sigma, M)$ is also a (measurable) eigenvalue for rotation by $\theta$; 
that is, $\exp (2 \pi i \delta)=\exp \left(2 \pi i k_{\theta}\right)$ for some $k$. Thus, if $\left(S_{n}^{x_{0}}-n \delta\right)$ is bounded, then $|I| \in \mathbf{Z} \theta$. A further application of this approach is made by Shapiro in extending a theorem of W. Schmidt. Let $\alpha$ be a sequence in $K$, and define $B(\alpha)$ to be the set of real numbers $\delta$ such that for some interval $I$ the sequence $\sum_{j=1}^{m}\left(\chi_{I}\left(\alpha_{j}\right)-\delta\right)$ is bounded. With the additional restriction that $\delta=|I|$, Erdös [44] conjectured $B(\alpha)$ is countable. This was proved by W. Schmidt, and the restriction $\delta=|I|$ removed by Shapiro:

1.13.7. Theorem (W. Schmidt [ 124] -[126]; L. Shapiro [130]). For any sequence $\alpha \subseteq K, B(\alpha)$ is at most countable.

I shall now digress with a definition of "prime flow."

1.13.8. Definition. A minimal flow $\mathcal{X}=(\Sigma, X)$ is prime if whenever $\mathcal{X} \rightarrow^{\pi}$ Y is a homomorphism, either $\mathcal{Y}$ is trivial or $\pi$ is an isomorphism (oneto-one, onto).

If $\Sigma$ is abelian, then for each $\sigma \in \Sigma, \sigma X$ is a closed invariant set, whence $\sigma X=X$ if $\mathfrak{X}$ is minimal. But then $\sigma$ also defines a homomorphism from $\mathfrak{X}$ to $\mathcal{X}$, and so if $\mathcal{X}$ is prime, $\sigma$ is one-to-one. Thus, we may as well assume $\Sigma$ is a group in the abelian case.

It is easy to construct prime flows. For example the homogeneous flows $(\mathbf{Z}, \mathbf{Z} / p \mathbf{Z}), p$ prime, and $\left(\operatorname{PSL}(2, \mathbf{R}), \mathbf{P}^{1}(\mathbf{R})\right)$ are prime. However the construction of a prime cascade $\mathscr{X}=(T, X)(\Sigma=\mathbf{Z}$ abelian) with $X$ infinite was done only recently by Furstenberg, Keynes, and Shapiro [51]. Some notations and definitions will be necessary.

Points $x, y \in X$ are proximal for $\mathcal{X}$ if the orbit closure of $(x, y)$ for $\mathscr{X} \times \mathfrak{X}$ contains a point of the diagonal. Write $x P y$ or $y P x$. It is not difficult to see that if $\mathscr{X}=(T, X)$ is minimal with $T$ invertible, if $X$ is infinite, and if $x P y$ whenever $x, y$ lie in distinct orbits, then $\mathcal{X}$ is prime. However, the question of whether such an $\mathcal{X}$ exists has not been answered (I believe). In [51] a less restrictive criterion for primeness is introduced:

1.13.9. Definition [51]. $\mathscr{X}$ is a POD-flow if (i) $\mathscr{X}$ is "totally minimal" $\left(T^{q}\right.$ is minimal for any $q \neq 0$ ), and (ii) for all $x \neq y$ there exists $n \neq 0$ such that $x P T^{n} y$.

\subsubsection{Proposition [51]. Every POD-flow is prime.}

The proposition is proved by noting that if $\mathfrak{X} \rightarrow^{\pi} \mathscr{Y}$ is not an isomorphism, then there exist $x \neq y$ with $\pi x=\pi y$. The POD property then implies $\{(z, w) \mid \pi z=\pi w\}$ contains the graph of $T^{n}$ for some $n \neq 0$, and therefore $T^{n}$ acts as the identity on $Y$. But $T^{n}$ must act minimally on $Y$, because it does on $X$, and therefore $\mathscr{Y}$ is trivial.

I shall give the main idea of the construction in [51], although the $T$ constructed below will not be continuous. A continuous one is constructed by "pulling points apart", i.e., passing to a symbol space.

Let $\theta \in K$ be irrational, and let $I \subseteq K$ be an interval with $|I| \notin \mathbf{Z} \theta$. Let $I^{\prime}$ be a second (distinct) copy of $I$, and let $\varphi: I \rightarrow I^{\prime}$ be the natural identification. Now let $X=K \cup I^{\prime}$, and define $T$ as 


$$
T x= \begin{cases}\varphi x, & x \in I, \\ \varphi^{-1} x+\theta, & x \in I^{\prime}, \\ x+\theta & \text { otherwise. }\end{cases}
$$

One computes that if $l=l(n, x)$ is the largest integer with $S_{l}^{x}+l \leqslant n$, then

$$
T^{n} x= \begin{cases}x+l \theta, & S_{l}^{x}+l=n, \\ \varphi(x+l \theta), & S_{l}^{x}+l=n-1 .\end{cases}
$$

The minimality of $T^{q}, q>0$, follows, for example, from Corollary 1.13.5. Now let $x, y \in K$ not belong to the same orbit under $T$, and consider the sequence

$$
(x+l(n, x) \theta)-(y+l(n, y) \theta)=(x-y+(l(n, x)-l(n, y)) \theta),
$$

$n \geqslant 1$. If this sequence has 0 for a cluster point, then (1.13.11) implies $x P T y, x P y$, or $x P T^{-1} y$, and property (ii) of POD follows easily. Now as $n$ increases by $1, l(n, x)-l(n, y)$ changes by 0 or \pm 1 . Therefore, if

$$
\sup _{n}|l(n, x)-l(n, y)|=\infty,
$$

the sequence $(l(n, x)-l(n, y)) \theta$ will be dense in $K$, and by the above we will be done. If the sup is finite, then $\sup \left|S_{l(n, x)}^{x}-S_{l(x, y)}^{y}\right|<\infty$, and these two facts imply $\sup _{l}\left|S_{l}^{x}-S_{l}^{y}\right|<\infty$. Since $x-y \notin \mathbf{Z} \theta$, this contradicts Theorem 1.12.13, and therefore $\mathcal{X}$ is POD. To understand the "continuous version" of X, see [51]. See also [78].

Since this section was written I have received a preprint by L. Shapiro [131] in which he extends his work and work of Schmidt on the torus to the torus, in the spirit of Theorem 1.13.7.

1.14. Interval exchange transformations. Let $\alpha=\left(\alpha_{1}, \ldots, \alpha_{n}\right)$ be a probability vector, and let $\tau$ be a permutation of $\{1,2, \ldots, n\}$. Define $\beta_{0}=0, \beta_{i}$ $=\sum_{j=1}^{i} \alpha_{j}, i \geqslant 1$. Denote by $\alpha^{\tau}$ the vector $\left(\alpha_{\tau^{-1} 1}, \alpha_{\tau^{-1} 2}, \ldots, \alpha_{\tau^{-1} n}\right)$, and form $\beta^{\tau}$ correspondingly. The $(\alpha, \tau)$ interval exchange $T$ is defined on $[0,1)$ as $T x=x-\beta_{(i)-1}+\beta_{\tau(2)-1}^{\tau}, x \in\left[\beta_{i-1}, \beta_{i}\right), 1 \leqslant i \leqslant n$. Note that $T$ is simply a right continuous, piecewise order preserving isometry of $[0,1)$. (See [3], [70], [134], [72].)

In case $n=\alpha$ and $\tau=(12)$, then $T x=x-\alpha_{1}(\bmod 1)$ is just a rotation. As $n$ increases, the interval exchanges grow rapidly more complicated. We say $T$ is minimal if every orbit is dense (even though $T$ is not continuous). The strongest minimality result is due to Keane:

1.14.1. THEOREM (M. KEANE [72]) . With notations as above, suppose $T^{m} \beta_{i}=T^{n} \beta_{j}$ implies $i=j$ and $m=n$. Then $T$ is minimal.

Keane calls his condition the "infinite distinct orbit" (i.d.o.) condition. It is satisfied, for example, if $(\alpha, \tau)$ is "irrational and irreducible", that is, if $\tau(1,2, \ldots, j)=(1,2, \ldots, j)$ implies $j=n$, and if $\alpha_{1}, \ldots, \alpha_{n}$ are independent over $Q$.

It was conjectured by Keane that every minimal interval exchange is ergodic with respect to Lebesgue measure, even uniquely ergodic, but this has proven to be false. (It is true for $n=2$ or 3 [72].) For $n=5$ Keynes and Newton [77] give an example in which minimality holds but ergodicity does not. In spirit, 
their example amounts to taking $\theta \in K$ irrational, $I$ an interval with $|I| \notin \mathbf{Z} \theta$, and noting that $T: K \times\{ \pm 1\} \rightarrow K \times\{ \pm 1\}$, defined by $T(x, \varepsilon)=(x+\theta, f(x) \varepsilon)$, where $f=-1$ on $I,=1$ on $I^{c}$, is isomorphic to a minimal interval exchange with $n=5$. If $\theta$ has unbounded partial quotients, and if $t \in \Gamma_{1 / 2}^{1}(2 \theta)(\S 1.12)$, then for some $I$ with $|I|=t$ or $1-t$, ergodicity will fail [149]. In turn, Keynes and Newton conjectured that if Lebesgue measure is ergodic, then $T$ is uniquely ergodic. But this is also false, as Keane [73] supplies a counterexample with $n=4$ (as small as possible). Keane's example is, in addition irrational.

REMARK. The fact that a minimal interval exchange can be ergodic without being uniquely ergodic actually follows from the Keynes-Newton example. For if $T$ is a minimal $(\alpha, \tau)$ interval exchange, and if $\mu$ is an invariant probability measure, then $\mu$ is nonatomic and assigns positive measure to every nonempty open set. Thus, $\varphi(x)=\mu([0, x))$ defines a homeomorphism of $[0,1)$, and defining $T_{1}$ by $T_{1} \varphi(x)=\varphi(T x), T_{1}$ is an $\left(\alpha^{1}, \tau\right)$ interval exchange, where $\alpha_{i}^{1}=\mu\left(\left[\beta_{i-1}, \beta_{i}\right)\right)$. Since $\varphi \mu$ is Lebesgue measure, Lebesgue measure is ergodic for $T_{1}$ if $\mu$ is ergodic for $T$. If $\mu$ is not unique for $T$, then $\varphi \mu$ is not for $T_{1}$, but $T_{1}$ is still minimal.

Keane [73] conjectures that if $\tau$ is an irreducible permutation, almost every $(\alpha, \tau)$ interval exchange is uniquely ergodic. The construction he employs in [73] suggests strongly that an auxiliary transformation on the "body" of interval exchanges will be of use in settling his conjecture. More precisely, let $\Delta_{n-1}$ be the simplex of probability $n$-vectors, and let $P_{n}^{*}$ be the set of irreducible permutations $\tau$ which in addition satisfy (a) $\tau(j+1) \neq \tau(j)+1$, all $j$, and (b) $\tau\left(\tau^{-1}(n)+1\right) \neq \tau(n)+1 .^{3}$ If $(\ddot{\alpha}, \tau) \in \Delta_{n-1} \times P_{n}^{*}$ satisfies the i.d.o. condition, and if $1 \leqslant i \leqslant n$, it is possible to prove the transformation induced on $\left[\beta_{i-1}, \beta_{i}\right)$ is an $\left(\alpha^{\prime}, \tau^{\prime}\right)$ interval exchange, $\left(\alpha^{\prime}, \tau^{\prime}\right) \in \Delta_{n-1} \times P_{n}^{*}$. Thus, we have a map $Q_{i}: \Delta_{n-1} P_{n}^{*} \rightarrow \Delta_{n-1} \times P_{n}^{*}$ defined a.e. If $\nu(\tau)=\operatorname{rank}\left\{E_{i j}\right.$ $\left.-E_{\tau(i) \tau(j)}\right\}$, where $E_{i j}=1$ for $j>i$ and 0 for $j \leqslant i$, it turns out that $\nu(\tau)$ $=\nu\left(\tau^{\prime}\right)$ above.

1.14.2. Problem. Is $Q_{i}$, restricted to the set $\left\{(\alpha, \tau) \in \Delta_{n-1} \times P_{n}^{*} \mid \nu(\tau)\right.$ $=$ const.\} ergodic?

1.14.3. Problem. Does $Q_{i}$ have an invariant measure equivalent to Lebesgue measure?

When $n=2$ or 3 (the cases in which the conjecture is already known), the answers to both questions are yes. For $n=2$ the measure is infinite, while for $n=3$ we do not yet know whether it is finite or infinite. Keane's conjecture would be true, for example, if the measure exists and is finite for $n \geqslant 4$.

Keane [71], [75] has established a connection between billiards on polygonal billiard tables whose corner angles are commensurable with $\pi$ and interval exchange transformations. I will not describe the connection here, but Keane does obtain, as a consequence of his minimality theorem, that for almost every (beginning) reflection angle from a fixed side, every trajectory is dense in the table. (See also [159].) Statements concerning the distribution of the trajectories could be made if one could prove the associated interval exchanges are ergodic, or better, uniquely ergodic.

\footnotetext{
${ }^{3}$ Also (c) $\tau\left(\tau_{1}^{-1}-1\right) \neq \tau 1-1,(\mathrm{~d})$ either $\tau_{n+1}^{-1} \neq \tau_{1}^{-1}$ or $\tau n+1 \neq \tau n$.
} 
It is interesting to note that when the table is square, certain skew products arise naturally. Identify one side, $L$, of the table with $(0,1)$, and denote a reflection from $L$ by position $(x)$ and angle of rebound $(\alpha)$, where $0<\alpha<\pi$. Beginning at $(x, \alpha)$ the next reflection from $L$ will have the form $\left(x^{\prime}, \alpha\right)$ or $\left(x^{\prime}, \pi-\alpha\right)$ (there is one $(x, \alpha)$ which ends up in a corner, and this we ignore). Now let $X=(0,1) \times\{\alpha, \pi-\alpha\}$, and define $T: X \rightarrow X$ by $T(x, \beta)=\left(x^{\prime}, \beta\right)$ or $\left(x^{\prime}, \pi-\beta\right)$ as above. For example, if $\alpha$ satisfies $1<\cot \alpha<\frac{3}{2}$, and if we set $\theta=2(\cot \alpha-1)$, it can be shown that $(T, X)$ is isomorphic to the skew product on $K \times\{ \pm 1\}$ defined using $\theta$ and $f=\mathfrak{X}_{(0, t)}-\mathfrak{X}_{(t, 1)}, t=1-\theta$. That is, $S(x, \varepsilon)=(x+\theta, f(x) \varepsilon),(x, \varepsilon) \in K \times\{ \pm 1\}$. (The correspondence is $(x, \alpha)$ $\sim(x, 1)$ and $(x, \pi-\alpha) \sim(1-x,-1)$.) Now even though $|I|=\theta$, it is proved in [149] that the skew product is uniquely ergodic (more precisely $\Re$ in the last section is uniquely ergodic) if $\theta$ is irrational (i.e., if $\cot \alpha$ is irrational). From this one easily infers that the trajectory of a ball bouncing off $L$ at an angle $\alpha$ is uniformly distributed on the table.

REMARK. Even in the case of a triangular table, little, if anything, seems to be known about density of trajectories if one of the corner angles is not commensurable with $\pi$.

Finally, I should mention that powerful techniques for dealing with "expansive" maps exist and may prove useful in connection with Problems 1.14.2 -1.14.4. See, for example, Sacksteder [120], Adler [1], Keane [74], and Walters [154].

\section{THE STRUCTURE OF MINIMAL FlOWS.}

While the notion of a minimal flow occurs in Birkhoff [12], it is only recently that efforts have been directed at uncovering the structure of arbitrary minimal flows. The first important structure theorem is the Furstenberg structure theorem for minimal "distal" flows [50], and this was followed by the structure of "point-distal" flows (Veech [146], Ellis [38]), and a structure theorem for arbitrary minimal flows (Ellis, Glasner and Shapiro [40], McMahon and $\mathrm{Wu}$ [90], McMahon [88]). In this section I shall prove a theorem, containing those above, which says roughly that the class of minimal flows (with fixed phase group) is the smallest class of flows containing the trivial flow and closed under (a) homomorphisms, (b) projective limits, and (c) three "building blocks" which will be specified later. It would be impossible to exaggerate the importance of the ideas and papers of Furstenberg and Ellis to the study of minimal flows.

2.1. Statements of results. In what follows $G$ will be a locally compact, $T_{2}$ topological group. Flows $\mathcal{X}=(G, X)$ will be assumed to satisfy the additional requirement that the map $L(g, x)=g x$ be continuous from $G \times X$ to $X$. Unless otherwise specified, $X$ is required to be compact, $T_{2}$ but not necessarily metrizable.

The Ellis separate continuity theorem [35] implies the following two statements, which will be important in later sections: (i) If $G$ is a locally compact $T_{2}$ space which is also a group, and if multiplication is left and right continuous, then $G$ is a topological group; (ii) in the first paragraph above, if $L(g, x)=g x$ is only assumed to be continuous in each variable separately, it 
is still jointly continuous. Namioka [96] has a nice generalization of the Ellis theorem with applications to other disciplines (e.g., Banach spaces).

A bitransformation group [34] is a triple $(G, X, H)$ such that (a) $(G, X)$ and $(H, X)$ are commuting flows ( $g h x=h g x, g \in G, h \in H, x \in X)$, (b) $H$ is compact, and (c) $(H, X)$ is strongly effective ("effective" means $h x=x$ for all $x$ implies $h=\mathrm{id}$; "strongly effective" means $h x=x$ for some $x$ implies $h=\mathrm{id}$ ). By (a) and (b) there is a natural flow $\mathcal{O}$ on $Y=X / H$. If $\mathfrak{X} \rightarrow^{\pi} \mathscr{Y}$ is the canonical homomorphism, then (c) implies for all $x \in X$ that $h \rightarrow h x$ defines a homeomorphism between $H$ and $\pi^{-1} \pi x$. $X \rightarrow^{\pi} \mathscr{Y}$ (or $\mathcal{X}$, or $\pi$ ) is called a group extension of $\mathscr{Y} . \mathfrak{X} \rightarrow \pi \mathscr{Y}$ is an almost periodic (a.p.) extension if there exist a group extension $\mathscr{Z} \rightarrow^{\pi^{\prime \prime}} \mathscr{Y}$ and a commutative diagram

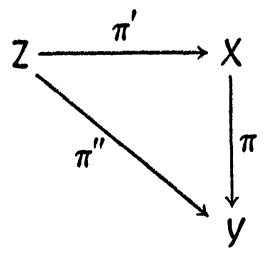

The trivial flow will be denoted $\Omega$. The equicontinuous flows are the a.p. extensions of $\Omega$.

$\mathcal{X} \rightarrow \pi \mathscr{O}$ defines an almost automorphic (a.a.) extension [146] or almost oneto-one extension if the set $A_{\pi}=\left\{y \mid \pi^{-1} y\right.$ is a singleton set $\}$ is nonempty $A_{\pi}$ is an invariant set, and therefore if $\mathcal{X}$ is minimal (resp. also metrizable), then $A_{\pi} \neq \varnothing$ implies $A_{\pi}, \pi^{-1} A_{\pi}$ are dense (resp. residual). To explain the terminology, call a point $x \in X$ an a.a. point for $\mathcal{X}$ if whenever $g_{\nu}$ is a net in $G$ such that $\lim _{\nu} g_{\nu} x=x^{\prime}$, then also $\lim _{\nu} g_{\nu}^{-1} x^{\prime}=x$. $X$ is an a.a. flow if there exists an a.a. point with dense orbit. Every a.a. flow is minimal, and the principal structure theorem is

2.1.2. THEOREM [138]. If $\mathcal{X}$ is an a.a. flow, there exist an equicontinuous flow $\mathcal{Y}$ and a homomorphism $\mathfrak{X} \rightarrow \pi$ Oु such that $\pi^{-1} A_{\pi}$ is the set of a.a. points. $\mathcal{X}$ is $a$ minimal equicontinuous flow if and only if $\mathcal{X}$ is minimal and every point is an a.a. point.

Theorem 2.1.2 is the basis for the main theorems about "almost automorphic" functions [138] which were introduced by Bochner [140].

$\mathcal{X} \rightarrow{ }^{\pi} \mathscr{Y}$ defines a proximal extension if $x P x^{\prime}(\$ 1.13)$ whenever $\pi x=\pi x^{\prime}$. If this proximality is uniform on $\pi^{-1} y$ for each $y$, in the sense that for every nonempty open set $U$ there exists $g \in G$ with $g \pi^{-1} y \subseteq U$, then $\mathcal{X} \rightarrow \pi \mathscr{Y}$ is highly proximal (h.p.) (McMahon and $\mathrm{Wu}$ [92], J. Auslander and S. Glasner [7]). Any minimal a.a. extension is h.p. (and proximal), while h.p. implies a.a.

if $X$ is metrizable [92], [7].

Let $X \rightarrow^{\pi}$ Y be a homomorphism of flows, and let $\alpha$ be an ordinal number. By a tower of flows of height $\alpha$ between $\mathcal{X}$ and $\mathcal{Y}$ we shall mean a set of flows and homomorphisms $\left\{\mathfrak{X}_{\beta} \rightarrow^{\pi_{\beta}} \mathfrak{X}_{\beta^{\prime}} \mid \beta^{\prime}<\beta \leqslant \alpha\right\}$ with the properties (a) $\mathfrak{X}_{0}$ $=\mathcal{Y}, \mathfrak{X}_{\alpha}=\mathcal{X}, \pi_{\alpha 0}=\pi$, (b) if $\beta^{\prime \prime}<\beta^{\prime}<\beta$, then $\pi_{\beta \beta^{\prime \prime}}=\pi_{\beta^{\prime} \beta^{\prime \prime}} \pi_{\beta \beta}$, and (c) if $\beta \leqslant \alpha$ is a limit ordinal, then $\lim _{\beta^{\prime}<\beta}^{-1} x_{\beta^{\prime}}=x_{\beta}$. Different kinds of towers lead to different names for the extension $\mathcal{X} \rightarrow \pi$ OY. 


$\begin{array}{ll}\text { Extension } & \text { Property of } \mathfrak{X}_{\beta+1} \rightarrow^{\pi_{\beta+1 . \beta}} \mathscr{X}_{\beta} \\ \text { I-extension [50] } & \text { a.p. for each } \beta<\alpha \\ \text { AI-extension [146] } & \text { a.p. or a.a. for each } \beta<\alpha \\ P I \text {-extension [40] } & \text { a.p. or proximal for each } \beta<\alpha \\ \text { HPI-extension [7] } & \text { a.p. or h.p. for each } \beta<\alpha\end{array}$

It is necessary to define one last kind of extension. Let $\mathscr{X} \rightarrow^{\pi} \mathscr{O}$ be a homomorphism, and define $R_{\pi}=\left\{\left(x, x^{\prime}\right) \mid \pi x=\pi x^{\prime}\right\}, \mathscr{R}_{\pi}=\left(G, R_{\pi}\right)$. $\mathscr{X} \rightarrow \rightarrow^{\pi}$ Q defines a weak mixing extension if $\Omega_{\pi}$ is topologically transitive (\$1.6). (When $X$ is metrizable, it is equivalent to say that $\Re_{\pi}$ has a point with dense orbit [63].)

What follows is a general structure theorem for homomorphisms of minimal flows. The construction of the diagram (2.1.4) is due to Ellis, Glasner and Shapiro [40] (see also McMahon and Wu) although in special cases it occurred previously in Veech [146] and Ellis [38]; the proof of the weak mixing statement is due to McMahon [88] for $X$ metrizable, while the general case is new.

2.1.3. TheOREM. Let $\mathfrak{X} \rightarrow^{\pi} \mathfrak{Y}$ be a homomorphism of minimal flows. There exist canonically determined minimal flows $x_{\alpha}$ and $\mathcal{Q}_{\alpha}$ and a commutative diagram

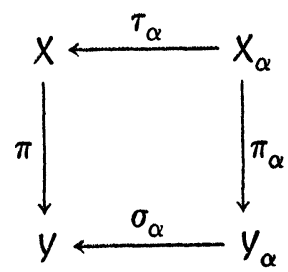

such that (a) $\mathfrak{X}_{\alpha}$ is a proximal extension of $\mathcal{X}$ and a weak mixing extension of $\mathscr{\vartheta}_{\alpha}$, and (b) $\mathcal{O}_{\alpha}$ is a PI extension of $\mathcal{Y}$. If $X$ is metrizable, $X_{\alpha}$ is metrizable.

Ellis, Glasner and Shapiro call $\mathscr{X}$ a "PPY flow" if when $\mathscr{Y}=\Omega, \pi_{\alpha}$ is an isomorphism in (2.1.4). In $\$ 2.7$ I will discuss some of their criteria for $\mathfrak{X}$ being (PS.

Let $\rightarrow^{\pi} \mathscr{O}$ be a homomorphism of minimal flows. A point $x \in X$ is $\mathcal{Y}$ distal ( $\pi$ is understood) if whenever $\left(x, x^{\prime}\right) \in R_{\pi}$ and $x P x^{\prime}$, then $x=x^{\prime}$. When

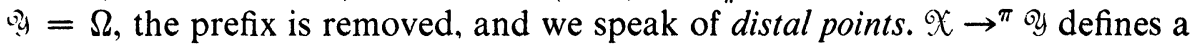
distal extension (distal flow if $\mathscr{Y}=\Omega$ ) if all points in $X$ are $\mathcal{Y}$-distal (distal). Distal flows were first studied by Ellis [33] who credits Hilbert with the notion. Certain results in [33], e.g., that a zero dimensional distal flow with finitely generated phase group is equicontinuous, led to the conjecture that distality implied equicontinuity. However, L. Auslander, Hahn and Markus [9] and Furstenberg [50] gave examples of minimal distal nonequicontinuous flows on certain compact nilmanifolds, thus proving the conjecture to be false. Perhaps the most important single breakthrough for the theorems under discussion was Furstenberg's structure theorem for minimal distal flows [50]. This theorem was extended by Ellis to (minimal) distal extensions [37], where began the "algebraic theory" of minimal sets. 
The notion of a distal flow was generalized by J. Auslander and Hahn [8] (see also Knapp [83]). We say $\mathfrak{X} \rightarrow^{\pi}$ $\mathcal{Y}$ defines $x$ as a $\mathscr{Y}$-point distal flow (point distal if $\mathscr{Y}=\Omega$ ) [146] if there exists a $\mathscr{Y}$-distal point with dense orbit. Assuming $\mathcal{O}$ is minimal, $\mathfrak{X}$ is also minimal (J. Auslander and Hahn [8], Knapp [83], Veech [146], but the argument goes back to Ellis [33]).

The structure theorem 2.1.5 below is due to Furstenberg for minimal distal flows [50], to Ellis for $\mathcal{Y}$-distal flows [37], to Veech [146] for $\mathcal{Y}$-point distal flows ( $\mathcal{Q}=\Omega$, but the general result was mentioned) having $X$ metrizable and a

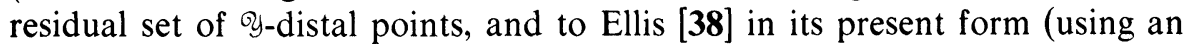
idea from Bronstein [20]). In the first two cases above of $\rightarrow^{\pi}$ oy is an Iextension, and so the $\mathfrak{X}_{\alpha}, \mathscr{Y}_{\alpha}$ in (2.1.4) do not enter. The idea of using a diagram such as (2.1.4) for a structure theorem occurs in [146].

2.1.5. TheOREM. Let $\mathcal{X} \rightarrow^{\pi}$ Oु be Oy-point distal, and assume either $G$ is $\sigma$ -

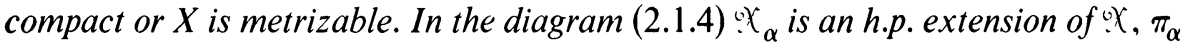
is an isomorphism, and $\mathcal{O}_{\alpha}$ is an HPI extension of $\mathcal{Y}_{\text {. If }} X$ is metrizable, replace "h.p." by "a.a." and "HPI" by "AI".

It was pointed out in [146] that the structure theorem implies that a 9 -point distal flow with metrizable phase space has a residual set of $\vartheta_{\text {-distal }}$ points. A direct proof of this fact has not been found.

The constructions of canonical towers in [146] and [38] on the one hand and in [40] on the other, though similar in spirit, are formally different. However, it was observed by J. Auslander and Glasner [7], using Ellis' structure theorem in [38], that the towers are in fact the same. In $\$ 2.3$ I shall give a direct proof of this fact.

Question. Is Theorem 2.1.5 true with no separability hypothesis? This is open for distal flows. It is not even known whether every nontrivial minimal distal flow has a nontrivial equicontinuous factor (a fact which is true for point distal flows with the separability assumption [50], [146]).

The techniques which will be developed in later sections are in part new and will be used to give a partial solution to a problem posed by Furstenberg. Let ox be a minimal cascade (homeomorphism) on a compact metric space, and assume $x$ has no nontrivial equicontinuous factor. Then $x \times$ is topologically transitive (Keynes and Robertson [79]). In fact, Furstenberg proves that if of is any minimal cascade, $\mathcal{X} \times$ o is topologically transitive. He raises the question [48] as to whether $\mathcal{X} \times \mathcal{O}$ is topologically transitive whenever of is topologically transitive. This is false in general; however we do have the following (as a special case of a more general result):

2.1.6. THEOREM. Let $x$ be an incontractible minimal flow, and assume $y$ has no nontrivial equicontinuous factor. Let of be a topologically transitive flow having a dense set of almost periodic points (e.g., a minimal flow). Then $x \times$ of is topologically transitive.

"Incontractible" will be defined in §2.3. (Every minimal flow with nilpotent phase group is incontractible.) A point $x$ is an almost periodic point (for $x)$ if $\mathfrak{Q}(x)$ is a minimal set. 
2.2. Generalities on flows. Denote by $e=e(G)$ the Banach algebra of bounded left uniformly continuous (l.u.c.) functions on $G$ and by $S=S(G)$ the maximal ideal space of $e$. The map $g \rightarrow s_{g}$ defined by $s_{g}(f)=f(g), f$ $\in \mathcal{C}$, embeds $G$ homeomorphically as an open dense subset of $S$. Often $g$ and $s_{g}$ will be identified. $S$ enjoys a universal property: if $\Lambda$ is a collection of maps from $G$ to some compact $T_{2}$ space, and if $\Lambda$ is jointly l.u.c., then $\Lambda$ has a jointly l.u.c. extension to $S$. If $K$ is a compact subset of $G$, and if $L(g, \cdot): G \rightarrow S$ is left multiplication by $g$, then $\Lambda_{K}=\{L(g, \cdot) \mid g \in K\}$ is jointly l.u.c. The extensions of $L(g, \cdot)$ to $S$ therefore define a flow $\delta(G)=\delta=(G, S)$. (We write $L(g, s)=g s$ interchangeably.)

For each fixed $s \in S$, the map $L(\cdot, s)$ extends to $S$ yielding a binary operation $L\left(s^{\prime}, s\right)=s^{\prime} s$ which is left continuous but not, in general, right continuous. The operation may be proved to be associative, and therefore $S$ is a compact $\left(T_{2}\right)$ semigroup with left continuous multiplication.

The reasoning above applies to any flow $\mathcal{X}$ so that $L(s, x)=s x$ is defined on $S \times X$ and continuous in the $S$ coordinate. Notice for any $x$ that $\mathcal{O}(x)=\overline{G x}=S x$, because $G$ is dense in $S$. (Applying this to $\left(x, x^{\prime}\right) \in X \times X$, we see that $x P x^{\prime}$ if and only if $s x=s x^{\prime}$ for some $s \in S$.) In particular, if $\mathscr{X}$ is point-transitive, meaning $\theta(x)=X$ for some $x$, the map $L(\cdot, x)$ defines a homomorphism from $\delta$ to $\mathcal{X}$. Thus $\S$ is the "universal" point transitive flow [34]. (Any point transitive flow of which $\delta$ is a factor is isomorphic to $\varsigma$, and therefore $\delta$ is unique up to isomorphism.) The following theorem is a classical result of Ellis [39] when $G$ is discrete. The general case will be proved in the Appendix.

2.2.1. THEOREM. If $G$ is a locally compact topological group, the flow $\$(G)$ is strongly effective.

If $\mathscr{X}$ is any flow the set $E(\mathcal{X})=\{L(s, \cdot) \mid s \in S\}$ may be regarded as a semigroup of transformations of $X$. With the point open (Tychonoff) topology, $E(X)$ is compact $T_{2}$ with multiplication left continuous. $E(X)$ contains a dense subgroup $\hat{G}$ of continuous maps (corresponding to $G$ ), and left multiplication by $\hat{G}$ is continuous on $E(\mathcal{X})$. $(\mathcal{E}=(G, E(X))$ is a flow defined by $L(g, s)=\hat{g} s$. One has $E(\mathcal{E}) \approx E(X)$ by a natural correspondence.) $E(X)$ is called the enveloping semigroup or Ellis semigroup of $x$. It is clear by the definition in terms of $S$ that if $x \rightarrow^{\pi}$ O is a homomorphism of flows, there is an induced

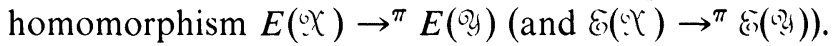

For the moment, let $E$ be a compact, $T_{2}$ semigroup with left continuous multiplication. Following Ellis [32] we note that if $\mathfrak{H}$ is the set of nonempty closed subsemigroups of $E$, then $\mathcal{H}$ contains a minimal element, $H$. If $x \in H$, then $H x \in \mathscr{H}$, meaning $H x=H$. In particular, $H_{0}=\{h \in H \mid h x=h\} \neq \varnothing$, and $H_{0} \in \mathcal{H}$. Thus, $H_{0}=H, x^{2}=x$, and $E$ contains an idempotent.

A nonempty set $I \subseteq E$ is an ideal if $E I \subseteq I$ (more properly left ideal, but there will be little occasion to consider right ideals). I is a minimal ideal if $I \neq \varnothing$ and if $I$ contains no proper ideal. As $E x$ is a closed ideal for any $x \in E$, and as $E x \subseteq I$ for any $x \in I, E x=I$ is closed if $I$ is a minimal ideal. Existence of minimal ideals is established by Zorn's lemma on the set of nonempty closed ideals. 
Let $I$ be a fixed minimal ideal. If $x \in I, I x$ is also an ideal, meaning $I x=I$, and $H=\{p \in I \mid p x=x\}$ is a closed subsemigroup. By the above, $H$ contains an idempotent $u$, and therefore if $J(I)=J$ is the set of all idempotents, $I=\cup_{u \in J} u I$. If $p \in u I$, and if $q \in I$ is such that $q p=u$, then $(u q) p=u$ also. As $u q \in u I$, it follows that $u I$ is a group with identity $u$. Now suppose $u, v \in J$ and $p \in u I \cap v I$. Choose $q \in u I$ with $p q=u$. Since $v p=p, u=v u=v\left(p^{\prime} u=p^{\prime}\right.$, all $p^{\prime} \in I$, because the set of such $p^{\prime}$ is an ideal containing $u$ ). Thus, $I$ is a disjoint union of groups (Ellis [32]).

REMARK. If $E$ contains a dense subgroup $\hat{G}$ which acts continuously, the minimal ideals in $E$ are the $\hat{G}$ minimal sets. In this setting it can be proved that if $I$ is minimal, and if $g \rightarrow^{\pi} g$ is a homomorphism of flows, then $\pi(p)=p \alpha$ for some fixed $\alpha \in I$ (J. Auslander [4]). Thus, $\pi$ is an isomorphism(Ellis [39]).

Let $\mathcal{X}$ be a minimal (in particular, point transitive) flow, and let $\delta \rightarrow^{\pi}$ o be a homomorphism. If $I \subseteq S$ is a closed nonempty ideal, $\pi I$ is an invariant closed set, hence all of $X$. In particular, if $I$ is a minimal ideal, there is a homomorphism $g \rightarrow^{\pi}$ X. The result mentioned in the last paragraph may be used to prove $G$ is unique up to isomorphism as a universal minimal set (Ellis [39]). Theorem 2.2 .1 implies $q$ is strongly effective.

Associate to $G$ two cardinals. The first, $a$, is defined to be 1 if $\left[G: G_{e}\right]$ is finite, where $G_{e}$ is the connected component of the identity, and otherwise $a=\operatorname{Card}\left[G: G_{e}\right]$. The second, $b$, is 1 if $G_{e}$ is compact, and otherwise $b=2^{c}$. It can be shown that $S$ contains $a b$ minimal ideals. If $G$ is discrete and $a>1$, then each minimal ideal has at least $2^{c}$ idempofents.

There are two useful characterizations of almost periodic (a.p.) points (points with minimal orbit closures): a "concrete" one due to Gottschalk and Hedlund [58], and an abstract one due to Ellis [32]. A set $L \subseteq G$ is left relatively dense (1.r.d.) if there exist $g_{1}, \ldots, g_{k} \in G$ with $\bigcup_{j=1}^{k} g_{j} L=G$.

2.2.2. Proposition. A point $x \in X$ is an a.p. point for $x$ if and only if whenever $U$ is a neighborhood of $x,\{g \in G \mid g x \in U\}$ is l.r.d.

2.2.3. Proposition. The following are equivalent for $x \in X$ :

(1) $x$ is an a.p. point for $X$.

(2) $x \in I x$ for any minimal ideal $I \subseteq E(X)($ or $\subseteq S)$.

(3) If I is a minimal ideal, then $x=u x$ for some $u \in J$.

REMARK. If $x, x^{\prime} \in X$, then by the last proposition $\left(u x, u x^{\prime}\right)$ is an a.p. point for $\mathfrak{X} \times \mathfrak{X}$. Therefore, if $u x P u x^{\prime}, u x=u x^{\prime}$ (it is impossible to leave the diagonal). Now suppose $x P x^{\prime}$ is a closed relation. If $x P x^{\prime}$, then as $\left(u x, u x^{\prime}\right)$ $\in \mathfrak{O}\left(x, x^{\prime}\right), u x P u x^{\prime}$. By the above $u x=u x^{\prime}$. This implies $P$ is an equivalence relation (Clay [22]). In general, $P$ is not an equivalence relation, and when it is, it may not be closed (Shapiro [129]).

REMARK. If $x \in X, u \in J$, then $u(x, u x)=(u x, u x)$, and so $x P(u x)$. If $x P y$ implies $x=y$, then $u x=x, u \in J$ (and conversely if $x$ is minimal; for if $v y=y, v \in J$, the fact $v x=x$ implies $v(x, y)=(x, y)$; thus $x P y$ implies $y=x)$.

Remark. The set $\{g \in G \mid g x \in U\}$ in Proposition 2.2.2 is a generalization of the translation numbers from Bohr's theory of almost periodic functions 
[14b]. (The idea of a translation number occurs at least as early as the thesis of E. Esclangon [45].)

2.3. The functor $2^{\mathscr{X}}$. If $X$ is compact, $T_{2}, 2^{X}$ will denote the space of nonempty closed subsets of $X . X$ has a unique compatible uniformity 2 which determines the Hausdorff uniformity $Q^{*}$. A base for $Q^{*}$ is the set of all $\alpha^{*}, \alpha \in \mathcal{Q}$, where $\alpha^{*}=\{(A, B) \mid B \subseteq \alpha[A]$ and $A \subseteq \alpha[B]\} .2^{X}$ is compact, $T_{2}$, and metrizable if $X$ is metrizable. Any flow $\mathcal{X}$ gives rise naturally to a flow $\left(G, 2^{X}\right)$ which we denote by $2^{x}$.

If $\mathcal{X} \rightarrow \pi$ Q is a homomorphism of minimal flows, $\pi_{\mathrm{ad}}: Y \rightarrow 2^{X}$ is defined by $\pi_{\mathrm{ad}} y=\pi^{-1} y . \pi_{\mathrm{ad}}$ is equivariant, upper semicontinuous and continuous if and only if $\pi$ is an open map. The construction to follow was introduced in [146] to circumvent certain difficulties which arise when $\pi$ fails to be open.

Assume $X$ is metrizable so that $2^{X}$ is also metrizable. The set $Y_{0}$ of points of continuity for $\pi_{\mathrm{ad}}$ is then invariant and residual. Let $Y^{*}=\overline{\pi_{\mathrm{ad}} Y_{0}} \subseteq 2^{X}$. $\mathrm{O}^{*}=\left(G, Y^{*}\right)$ is a flow, and there is a natural homomorphism Of $^{*}$ $\rightarrow^{\sigma \mathcal{O}}\left(\sigma \pi_{\mathrm{ad}} y=y, y \in Y_{0}\right)$. The minimality of $\mathcal{Y}$ and continuity of $\pi_{\mathrm{ad}}$ at points of $Y_{0}$ imply (a) $\mathcal{O}^{*}$ is minimal, and (b) $\sigma^{-1} y=\left\{\pi_{\mathrm{ad}} y\right\}, y \in Y_{0}$, i.e., $\mathcal{Y}^{*}$ is an a.a. extension of $\mathscr{Y}$.

Now define $X^{*} \subseteq X \times 2^{X}$ by $X^{*}=\left\{(x, A) \mid x \in A \in Y^{*}\right\}$. Set $\mathcal{X}^{*}=(G$, $X^{*}$ ) and let $\mathfrak{X}^{*} \rightarrow^{\tau} \mathfrak{X}$ and $\mathfrak{X}^{*} \rightarrow^{\pi^{*}} \mathcal{Y}^{*}$ be the coordinate maps. It is routine to check that $\mathfrak{X}^{*}$ is a minimal a.a. extension of $\mathcal{X}$ and $\pi^{*}$ is an open map. Of course $X^{*}$ is metrizable, and the diagram

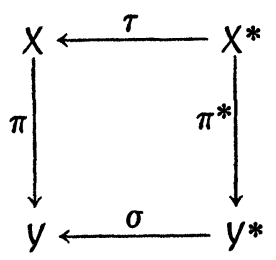

is commutative.

A fruitful generalization of the "shadow diagram" (2.3.1) was made by Ellis, Glasner and Shapiro [40] (see also McMahon and $\mathrm{Wu}$ [90]). In what follows $X$ is not assumed to be metrizable. If $A \in 2^{X}, s \in S(G)$, then $s A$ has two possible interpretations, either $s A=\{s a \mid a \in A\}$, which may not be closed, or $s A$ is the image of the "point" $A \in 2^{X}$ under $s$ which is a closed set (i.e. another point of $2^{X}$ ). Following [40] we denote the latter by $s \circ A$. Always $s A \subseteq s \circ A$, but usually they are distinct. If $A \subseteq X$ is an arbitrary set, $s \circ A$ is understood to be $s \circ \bar{A}$.

Let $I \subseteq S$ be a minimal ideal, and let $J=J(I)$. (The construction below is in fact independent of the choice of $I$.) If $y \in Y$, define $I_{y}=\{p \in I \mid p y=y\}$ and $J_{y}=J\left(I_{y}\right)$. We have $I_{y}=\cup_{u \in J_{y}} u I_{y}$ (disjoint union of groups). For each $u \in J_{y}$ define $C_{\pi}(y, u)=C(y, u) \stackrel{=}{=} \circ\left(u \pi^{-1} y\right)$. As a set, $C_{\pi}(y, u) \subseteq \pi^{-1} y$, and as a point (in $\left.2^{X}\right) C_{\pi}(y, u)$ has a minimal orbit closure. One may prove (cf. [40]) that if $p \in I, v \in J$ satisfy $v p=p$, then $p \circ C_{\pi}(y, u)=C_{\pi}(p y, v)$. It follows that

$$
Y^{\prime}=\mathcal{O}\left(C_{\pi}(y, u)\right)=I \circ C_{\pi}(y, u)=\left\{C_{\pi}\left(y^{\prime}, v\right) \mid y^{\prime} \in Y, v \in J_{y^{\prime}}\right\} .
$$


$\mathcal{Y}^{\prime}$ is a minimal flow, and if $\sigma^{\prime}\left(C_{\pi}\left(y^{\prime}, v\right)\right)=y^{\prime}, \mathscr{Y}^{\prime} \rightarrow^{\sigma^{\prime}}$ OY is a homomorphism of minimal flows. If $y \in Y, u, v \in J_{y}$, the discussion above implies $u$ $\circ C_{\pi}(y, v)=C_{\pi}(y, u)=u \circ C_{\pi}(y, u)$, and, therefore, $C_{\pi}(y, v) P C_{\pi}(y, u)$. That is, $\mathcal{O}^{\prime}$ is a proximal extension of $\mathcal{Y}$.

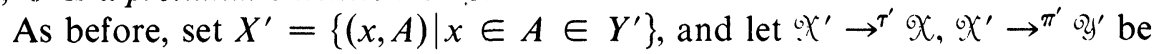
the natural maps. $\mathfrak{X}^{\prime}$ is a minimal proximal extension of $\mathcal{X}$ and the map $\pi^{\prime}$ is open. Of course the shadow diagram

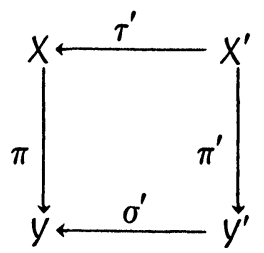

is commutative. The advantage of $\left(2.3 .1^{\prime}\right)$ over (2.3.1) (in some cases they coincide, see below) is that $\pi^{\prime}$ enjoys a somewhat stronger property than simply being open. For if $y^{\prime} \in Y^{\prime}$, say $y^{\prime}=C_{\pi}(y, u)$, then $\pi_{\text {ad }}^{\prime} y^{\prime}=C_{\pi}(y, u)$ $\times\left\{C_{\pi}(y, u)\right\}$. Therefore, $C_{\pi^{\prime}}\left(y^{\prime}, u\right)=\pi_{\mathrm{ad}}^{\prime} y^{\prime}$. This brings us to

2.3.2. Definition (Ellis, Glasner and Shapiro [40]). Let $\mathscr{X} \rightarrow^{\pi}$ OY be a homomorphism of minimal flows. We say $\pi$ defines an RIC extension (relatively incontractible) if $C(y, u)=\pi_{\mathrm{ad}} y$ for all $y \in Y, u \in J_{y}$. ox is incontractible if it is an RIC extension of $\Omega$.

In $\left(2.3 .1^{\prime}\right) \pi^{\prime}$ defines an RIC extension; $\pi$ is RIC there if and only if $\sigma^{\prime}, \tau^{\prime}$ are isomorphisms.

2.3.3. Definition. We say $\mathfrak{X} \rightarrow^{\pi} \mathscr{Y}$ satisfies the Bronstein condition if $R_{\pi}$ contains a dense set of a.p. points.

The significance of Bronstein's condition [20] will be made clear in \$2.6. If $\pi$ is an RIC extension, then $G\left(u \pi^{-1} y \times u \pi^{-1} y\right), u \in J_{y}$, is a dense set of a.p. points in $R_{\pi}$, and therefore $\pi$ satisfies the Bronstein condition. It also follows that in $\left(2.3 .1^{\prime}\right) \tau \times \tau\left(R_{\pi^{\prime}}\right)$ is the closure of the set of almost periodic points it contains, because if $z^{\prime} \in R_{\pi^{\prime}}$ is a.p., $\tau \times \tau\left(z^{\prime}\right)$ is a.p. On the other hand, if $z=\left(x_{1}, x_{2}\right) \in R_{\pi}$ is an a.p. point, say $u z=z$, then $x_{1}, x_{2} \in C(y, u), \pi x_{1}$ $=y$. Thus,

$$
\left(x_{1}, x_{2}\right)=\tau \times \tau\left(\left(x_{1}, C(y, u)\right),\left(x_{2}, C(y, u)\right)\right),
$$

and so $\tau \times \tau\left(R_{\pi^{\prime}}\right)$ is the closure of the a.p. points in $R_{\pi}$. In particular,

2.3.4. Lemma. Let $\mathfrak{X} \rightarrow^{\pi} \mathscr{Y}$ satisfy the Bronstein condition. In (2.3.1') we have $\tau \times \tau\left(R_{\pi^{\prime}}\right)=R_{\pi}$.

A more direct generalization of (2.3.1) has been given by $\mathbf{J}$. Auslander and Glasner [7]. With notations as above, set $Y^{*}=I \circ \pi_{\mathrm{ad}} y, X^{*}=\{(x, A) \mid$ $\left.x \in A \in Y^{*}\right\}$. If $X$ is metrizable, then $Y^{*}, X^{*}$ coincide with the objects in (2.3.1). In any case (2.3.1) is again defined (and independent of $y, I$ ), and $\pi^{*}$ is an open map. This time, however, $\sigma$ and $\tau$ define h.p. extensions but possibly not a.a. extensions. (Given $y \in Y$, Zorn's lemma and the fact $\sigma_{\mathrm{ad}} y \in 2^{2^{x}}$ is used to prove there exists $A \in \sigma_{\mathrm{ad}} y$ such that if $B \in \sigma_{\mathrm{ad}} y, B \subseteq A$, then $B=A$. Choose $p \in I$ with $p \circ \pi^{-1} y=A$. It is straightforward to check that $p \circ \sigma_{\text {ad }} y=\{A\}$, and $\sigma$ is h.p. Similarly for $\tau$.) 
In the structure theorem for point-distal extensions it is h.p. rather than proximal extensions which play a role. Since it is more convenient to work with $\left(2.3 .1^{\prime}\right)$, it is natural to ask for a condition guaranteeing that (2.3.1) and $\left(2.3 .1^{\prime}\right)$ are identical (i.e., $\mathcal{Y}^{*}=\mathscr{Y}^{\prime}, X^{*}=x^{\prime}$, etc.). I shall prove

2.3.5. TheOREM. Let $\mathfrak{X} \rightarrow^{\pi}$ OY be a homomorphism of minimal flows, and assume either $X$ is metrizable or $G$ is $\sigma$-compact. A necessary and sufficient condition for $\left(2.3 .1^{\prime}\right)$ and (2.3.1) to coincide is that for some $y \in Y, \cap_{u \in J_{y}} C(y, u)$ $\neq \varnothing$.

The theorem provides an intrinsic proof of a result of $\mathrm{J}$. Auslander and Glasner [7] which was proved using the structure theorems themselves.

2.3.6. Corollary. Let $\mathcal{X} \rightarrow^{\pi}$ Oy be such that there is a $\mathcal{Y}$ distal point, and assume either $X$ is metrizable or $G$ is o-compact. Then (2.3.1) and (2.3.1') coincide.

PROOF. Let $x$ be $y$-distal, the set $\pi x=y$. If $u \in J_{y}$, then because $x P u x$, and $\pi u x=y$, we have $x=u x$. Therefore, $x \in C(y, u)$. Since $u$ is arbitrary, $x \in \cap_{u \in J_{y}} C(y, u)$, and Theorem 2.3.5 applies.

I shall prove Theorem 2.3 .5 only in the metrizable case. The extension to $\sigma$ compact $G$ is made by "approximating" $\mathcal{X} \rightarrow^{\pi}$ ? $\mathrm{Y}$ by homomorphisms of minimal flows with metrizable phase spaces. The theorem turns on the following

2.3.7. Proposition. Let $\mathfrak{X} \rightarrow^{\pi}$ oy be a homomorphism of minimal flows, and let $X$ be metrizable. Let $\varphi: Y \rightarrow 2^{2^{X}}$ be an equivariant Borel map such that (a) $\varphi(y) \in 2^{2^{\pi^{-1}} y}, y \in Y$, and (b) $Y_{\varphi}=\left\{y \in Y \mid \cap_{A \in \varphi(y)} A \neq \varnothing\right\}$ is a second category set. Then for some $y \in Y, \varphi(y)=\left\{\pi^{-1} y\right\}$.

I shall first derive the theorem from the proposition. Define $\varphi(y)=\{C(y, u) \mid$ $\left.u \in J_{y}\right\}$. Then $\varphi$ is u.s.c., hence Borel. If we assume $Y_{\varphi} \neq \varnothing$, then $Y_{\varphi}$, being invariant, is dense. It follows easily that if $y$ is a point of continuity of $\varphi$, then $y \in Y_{\varphi}$. As the points of continuity comprise a residual set, $Y_{\varphi}$ is residual. By the proposition $\varphi(y)=\left\{\pi^{-1} y\right\}$ for some $y$, or $\pi^{-1} y=C(y, u), u \in J_{y}$, for some $y$. Thus, $Y^{\prime} \cap Y^{*} \neq \varnothing$, and being (invariant) minimal sets, they must coincide.

To prove the proposition, define $D: 2^{2^{X}} \rightarrow 2^{X} \cup\{\varnothing\}$ by $D(\lambda)=\cap_{A \in \lambda} A$. $D(\cdot)$ is u.s.c., hence Borel, and therefore $\psi(y)=D(\varphi(y))$ is a Borel map from $Y$ to $2^{X} \cup\{\varnothing\}$. Since $Y$ and $2^{X} \cup\{\varnothing\}$ are compact metrizable and $\psi$ is Borel, there exists a residual set $Y_{\psi} \subseteq Y$ such that $\left.\psi\right|_{Y_{\downarrow}}$ is continuous. The equivariance of $\psi$ implies that $Y_{\psi}$ may be assumed to be invariant. (See below.) Now $Y_{\psi} \cap Y_{\varphi} \neq \varnothing$, because $Y_{\varphi}$ is second category, and therefore $Y_{\psi} \cap Y_{\varphi}$ is dense in $Y_{\psi}$. Since $\varnothing$ is an isolated point of $2^{X} \cup\{\varnothing\}, Y_{\psi} \cap Y_{\varphi}=Y_{\psi}$. Now let $y \in Y_{\psi}$ be any point of continuity for $\pi_{\mathrm{ad}} . G \psi(y)$ is a dense subset of $X$ by minimality, and therefore $\lim _{\sup _{g y \rightarrow y}} \psi(g y)=\pi^{-1} y$. By continuity, $\psi(y)$ $=\pi^{-1} y$, and therefore $\varphi(y)=\left\{\pi_{\mathrm{ad}} y\right\}$ as claimed.

RemarK. An observation by Ellis [38] is useful for an iteration which will be carried out later using $\left(2.3 .1^{\prime}\right)$. It is that if $x \in X$ is a $\mathcal{Y}$-distal point, then for any $A \in Y^{\prime}$ such that $x \in A,(x, A) \in X^{\prime}$ is a $\mathcal{Y}^{\prime}$ distal point. For suppose 
$\left(x^{\prime}, A^{\prime}\right) \in\left(\pi^{\prime}\right)^{-1} A$. Then $A^{\prime}=A$, and if $x^{\prime} P x, x^{\prime}=x$. Thus, $\left(x^{\prime}, A^{\prime}\right) P(x, A)$ implies $\left(x^{\prime}, A^{\prime}\right)=(x, A)$.

The invariance of $Y_{\psi}$ used in the proof of the proposition may be obtained from the following lemma.

2.3.8. LemMa. Let $\mathscr{W}, \mathscr{Z}$ be flows with metric phase spaces $\left(W, D_{W}\right),\left(Z, D_{Z}\right)$, and let $f: W \rightarrow Z$ be an equivariant map. If $A \subseteq W$ is a residual set such that $\left.f\right|_{A}$ is continuous, then also $\left.f\right|_{G A}$ is continuous.

Proof. Suppose $\lim _{k} g_{k} a_{k}=g a, a_{k}, a \in A$. Replacing $g_{k}$ by $g^{-1} g_{k}$ and using equivariance of $f$ and continuity of $g$ we may suppose $g=e$. Because $A$ is residual, $A_{k}=A \cap g_{k}^{-1} A$ is residual for each $k$, and we may choose $b_{k} \in A_{k}$ such that (i) $D_{W}\left(g_{k} b_{k}, g_{k} a_{k}\right)<1 / k$, (ii) $D_{Z}\left(g_{k} f\left(b_{k}\right), g_{k} f\left(a_{k}\right)\right)$ $<1 / k$. By (i) $\lim _{k} g_{k} b_{k}=a$, and since $a, g_{k} b_{k} \in A, \lim _{k} f\left(g_{k} b_{k}\right)=f(a)$. By (ii), $\lim _{k} f\left(g_{k} a_{k}\right)=\lim _{k} g_{k}\left(f\left(a_{k}\right)\right)=f(a)$ also.

The following result is another consequence of Proposition 2.3.7.

2.3.9. Proposition. Let $\mathfrak{X} \rightarrow \rightarrow^{\pi}$ Oु be a homomorphism of minimal flows with $X$ metrizable, and let $A \subseteq X$ be an invariant Borel set such that $\lambda(y)=A \cap \pi^{-1} y$ is closed and nonempty for each $y$. Then

(i) if $\lambda(y)$ is a singleton for each $y, \pi$ is an a.a. extension,

(ii) if $\lambda(\cdot)$ is a Borel function from $Y$ to $2^{X}$, then $\lambda(y)=\pi^{-1} y$ for some $y$.

In (i), let $\lambda(y)=\{f(y)\}$. If $B \subseteq X$ is Borel, $f^{-1} B=\pi(B \cap A)$ is a continuous one-one image of a Borel set, hence Borel. Thus (i) follows from (ii). Of course (ii) follows from Proposition 2.3.7 using $\varphi(y)=\{\lambda(y)\}$.

2.4. Almost periodic extensions. Let $\mathfrak{X} \rightarrow \pi$ $\mathscr{Y}$ be a homomorphism of minimal flows, and let $थ$ be the compatible uniformity for $X$. Define $P_{\pi} \subseteq R_{\pi}$ by $P_{\pi}=\bigcap_{\alpha \in \mathcal{Q}}\left(G \alpha \cap R_{\pi}\right)$. $P_{\pi}$ is called the relative proximal relation (proximal relation if $\mathcal{Y}=\Omega$ ). The argument for Clay's result $(\$ 2.2$, see [22]) shows that if $P_{\pi}$ is closed, it is an equivalence relation. As mentioned earlier, $P_{\pi}$ is generally not an equivalence relation, and when it is, it is generally not closed. Of course, $G P_{\pi}=P_{\pi}$.

Now assume $\mathcal{X}$ is a distal extension of $\mathcal{O}\left(P_{\pi}=\Delta\right)$. If $y \in Y, u \in J_{y}$, then $u x=x, x \in \pi^{-1} y$ (see Corollary 2.3.6). Fix $y$ and set up $\mathcal{W}=\prod_{x \in \pi^{-1} y} \mathfrak{x}_{x}$, $\mathcal{X}_{x}=\mathcal{X}$. Define $z \in W$ by $z(x)=x, x \in \pi^{-1} y$. Clearly, $u z=z, u \in J_{y}$, and so $Z=\vartheta(z)$ is a minimal set (Proposition 2.2.3). There is a natural commutative diagram

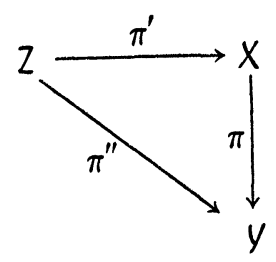

Let $p, p^{\prime}, q \in I$ be such that $p z=p^{\prime} z$ and $q y=y$. For each $x \in \pi^{-1} y, q x$ is a coordinate of $z$ (the $q x$ th) and therefore $p q x=p^{\prime} q x$. That is, $p q z=p^{\prime} q z$, and we have a natural pairing $Z \times I_{y} \rightarrow Z$ defined by $L(p z, q)=p q z$. If $q \in I_{y}$, $u q=q, u \in J_{y}$, choose $q^{\prime} \in u I_{y}$ with $q q^{\prime}=u$. Then $L\left(L(p z, q), q^{\prime}\right)=p q q^{\prime} z$ 
$=p z$, and so $L(\cdot, q)$ is invertible. It is also continuous, and so if we let $H$ be the set of maps $\left\{L(\cdot, q) \mid q \in I_{y}\right\},(H, Z)$ is a "flow" (except that $H$ may not be a topological group). Note that the actions of $G$ and $H$ on $Z$ commute, and $\mathcal{Y}=\mathscr{Z} / H$. Moreover, $H$ acts strongly effectively. For if $p q z=p z$, the $\mathcal{Y}$ distality of $\mathfrak{X}$ implies $q x=x, x \in \pi^{-1} y$. That is, $q=\mathrm{id}$ in $H$. Ellis calls (2.4.1) a "group-like extension" [37].

Now define $Q_{\pi}=\cap_{\alpha \in \mathcal{Q}} \overline{G \alpha \cap R_{\pi}} \cdot Q_{\pi} \supseteq P_{\pi}$ is the relative regionally proximal relation (regionally proximal relation if $\mathcal{O}=\Omega$ ). If $Q_{\pi}=\Delta$, then $P_{\pi}=\Delta$, and (2.4.1) is defined. But this time one finds that (a) $H$ has separately continuous multiplication and is therefore a topological group, and (b) the pairing $(H, Z)$ is separately continuous and therefore a flow. In other words, if $Q_{\pi}=\Delta, \mathscr{Z}$ is a group extension of $\mathscr{Y}$ and $\mathscr{X}$ an a.p. extension. Conversely, if $\mathfrak{X}$ is an a.p. extension of $\mathscr{Y}$, one sees that $Q_{\pi}=\Delta$.

The first a.p. extensions were the isometric extensions introduced by Furstenberg [50]. What follows is a straightforward generalization of Furstenberg's notion:

2.4.2. Definition. Let $\mathfrak{X} \rightarrow^{\pi}$ Y be a homomorphism of minimal flows. $\mathfrak{X}$ shall be called an F-extension of $\mathscr{Y}$ if for each $z \in R_{\pi}-\Delta$ there exists $\delta \in C\left(R_{\pi}\right)$ such that
(a) $\delta \geqslant 0,\left.\delta\right|_{\Delta}=0$,
(b) $G \delta=\delta$,
(c) $\delta(z)>0$.

If $\mathscr{X}$ is an $F$-extension of $\mathscr{Y}$, then $Q_{\pi}=\Delta$. For if $z \notin \Delta$, choose $\delta \in C\left(R_{\pi}\right)$ as in (a) -(c), and let $\alpha \in \mathcal{Q}$ be such that $\alpha \cap R_{\pi} \subseteq\left\{z^{\prime} \mid \delta\left(z^{\prime}\right)<\frac{1}{2} \delta(z)\right\}$. Then $z \notin \overline{G \alpha \cap R_{\pi}}$, meaning $z \notin Q_{\pi}$. Conversely, if $x$ is an a.p. extension of $\mathscr{Y}$, $\mathcal{X}$ is an $F$-extension. This is not a difficult result and will not be proved here. It follows from more difficult results in the next section. We have

2.4.3. Proposition. Let $\mathfrak{X} \rightarrow{ }^{\pi} \mathcal{Y}$ be a homomorphism of minimal flows. The following are equivalent:

(i) $\mathcal{X}$ is an a.p. extension of $\mathcal{O}$,

(ii) $Q_{\pi}=\Delta$,

(iii) $\mathcal{X}$ is an F-extension of oु.

Let $E_{\pi}$ be the least closed invariant equivalence relation which contains $Q_{\pi}$. $\mathcal{X} / E_{\pi}$ is the largest almost periodic extension of $\mathcal{Y}$ "below" $\mathcal{X}$. When $\mathcal{X}=\mathscr{g}, \mathscr{g} / E_{\pi}$ is the largest minimal a.p. extension of $\mathcal{Y}$, in the sense that any other minimal a.p. extension of $\mathcal{Y}$ is a factor of $q / E_{\pi}$.

REMARK. Let $\mathscr{W}$ be the largest minimal a.p. extension of $\mathcal{Y}$, and let $\mathcal{X} \rightarrow^{\pi}$ Oु, $\mathscr{Z}=\mathcal{X} / E_{\pi}$ be as above. There is a commutative diagram

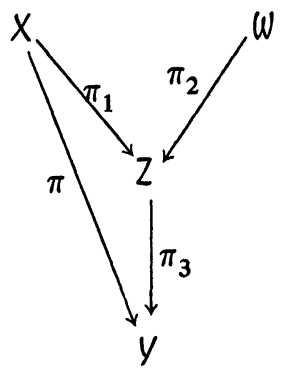


Define $R_{\pi_{1} \pi_{2}}=\left\{(x, w) \mid \pi_{1} x=\pi_{2} w\right\}$. Then it is possible to show $\Re_{\pi_{1} \pi_{2}}$ is itself a minimal flow. (For $\mathcal{Y}=\Omega, G$ abelian this is proved (and used) in [150].)

2.5. Topologies associated to minimal flows. The classical characterization of distality, due to Ellis [33], states that $\mathcal{X}$ is a distal flow if and only if $E(X)$ is a group. This played a major role in Furstenberg's structure theorem for minimal distal flows (and also contributed to the early false conjecture that distal flows are equicontinuous).

Let $\mathcal{X}$ be a minimal distal flow with (for now) metrizable phase space. Furstenberg's idea is to endow $X$ with a weak(er) topology with respect to which the elements of $E(X)$ are homeomorphisms. With the weak(er) pointopen topology $E(\mathfrak{X})$ has multiplication separately continuous (and inversion continuous). Should either of the weak topologies be $T_{2}$, they both are and (a) $E(\mathcal{X})$ is a topological group in its "regular" topology, and (b) $\mathscr{X}$ is an equicontinuous flow. In general, the weak topologies are $T_{1}$ but not $T_{2}$, and so Furstenberg employs an ingenious fiberwise "Hausdorffization" to prove that if $\mathcal{X} \rightarrow \pi \mathscr{Y}$ is a nontrivial extension of $\mathcal{O}$, it is possible to "insert" a nontrivial a.p. extension of $\mathscr{Y}$ below $\mathfrak{X}$. A transfinite induction, beginning with $\mathscr{Y}=\Omega$, proves $\mathcal{X}$ is an $I$-flow.

REMARK. The condition that a group $K$ be compact, with multiplication separately continuous and inversion continuous, occurs so frequently that I shall refer to $K$ simply as an " $F$-group."

A different procedure for defining weak topologies was given by Ellis [37] and used by him to prove his structure theorems [37], [38]. The idea of attaching "Furstenberg topologies" to point-distal flows occurs in [146], where Hausdorffization and shadow diagrams were used to prove the structure theorem. Below I shall sketch the (natural generalization of the) Furstenberg topologies of [146] and prove they are equivalent to Ellis' topologies. The latter fact has been proved independently by Ellis, Glasner and Shapiro [40].

Fix a minimal flow $\mathcal{X}$, and let $\Sigma$ be the set of continuous pseudometrics on $X$. If $\sigma \in \Sigma$ define $F_{\sigma}$ on $X \times X$ by $F_{\sigma}(z)=\inf _{g \in G} \sigma(g z)$. $F_{\sigma}$ is invariant and u.s.c., which implies for all $z \in X \times X$ (resp. all a.p. $z \in X \times X$ ) and $p \in S$ or $E(\mathfrak{X})$,

$$
F_{\sigma}(z) \leqslant F_{\sigma}(p z) \quad\left(\text { resp. }=F_{\sigma}(p z)\right) .
$$

If $x \in X, \sigma \in \Sigma, \varepsilon>0$, define $U_{\varepsilon, \sigma}(x)=\left\{x^{\prime} \mid F_{\sigma}\left(x, x^{\prime}\right)<\varepsilon\right\} . U_{\varepsilon, \sigma}$ is open because $F_{\sigma}$ is u.s.c. The following lemma is basic and is due to Furstenberg [50]; see also [146].

2.5.2. LemmA. If $u \in J, z=\left(x_{1}, x_{2}\right)$ are such that $u z=z$, and if $\sigma \in \Sigma, \varepsilon$ $>0$ are such that $F_{\sigma}(z)<\varepsilon$, then there exist $\rho \in \Sigma$ and $\delta>0$ such that $U_{\delta, \rho}\left(x_{2}\right) \subseteq U_{\varepsilon, \sigma}\left(x_{1}\right)$.

REMARK. If $x_{2}^{\prime} \in X$ and $u x_{2}^{\prime}=x_{2}$, then $F_{\rho}\left(x_{2}, x_{2}^{\prime}\right)=0$. Therefore $x_{2}^{\prime}$ $\in U_{\varepsilon, \sigma}\left(x_{1}\right)$. (This also follows from (2.5.1) directly.) Define $U_{\varepsilon, \sigma}^{u}(x)=U_{\varepsilon, \sigma}(x)$ $\cap X(u)$.

The lemma implies the set $\mathfrak{F}_{0}(u)=\left\{U_{\varepsilon, \sigma}^{u}(x) \mid x \in X(u), \varepsilon>0, \sigma \in \Sigma\right\}$ is a basis for a topology $\mathfrak{F}(u)$ on $X(u)$. If $x, x^{\prime}$ are distinct points of $X(u)$ the fact that $x$ and $x^{\prime}$ cannot be proximal implies $F_{\sigma}\left(x, x^{\prime}\right)>0$ for some $\sigma$, and 
therefore $\mathfrak{F}(u)$ is $T_{1}$. The remark following Lemma 2.5.2 implies any open cover $\theta=\left\{U^{u}\right\}$ from $\mathfrak{F}_{0}(u)$ corresponds to an open cover $\vartheta^{\prime}=\{U\}$ of $X$, and therefore has a finite subcover. That is, $(X(u), \mathfrak{F}(u))$ is compact.

Each $\gamma \in u I(I$ a minimal ideal in $S$ or $E(X))$ maps $X(u)$ to itself and by (2.5.1) is an " $\mathfrak{F}(u)$ isometry". Therefore $\gamma$ is an $\mathfrak{F}(u)$ homeomorphism. Let $K(u)=u I$, and place on $K(u)$ the point-open $(\mathfrak{F}(u))$ topology, $\mathfrak{F}^{*}(u) \cdot \mathfrak{F}^{*}(u)$ is compact and $T_{1}$ if $I \subseteq E(\mathfrak{X})$, but usually not $T_{1}$ if $I \subseteq S$ (because the action of $K$ on $X(u)$ may not be effective if $I \subseteq S)$. Multiplication is separately continuous, and, because $F_{\sigma}(x, \gamma x)=F_{\sigma}\left(x, \gamma^{-1} x\right), x \in X(u), \gamma \in K(u)$, inversion is continuous. That is, $\left(K(u), \mathfrak{\Im}^{*}(u)\right)$ is an $F$-group.

REMARK. It is not true in general that $X(u)$ is $G$ invariant or that $G$ has a natural homomorphism into $K(u)$. These facts are of surprisingly little consequence.

REMARK. If $x \in X(u)$, define $\lambda_{x}: K(u) \rightarrow X(u)$ by $\lambda_{x}(\gamma)=\gamma x$. By definition of $\mathfrak{F}^{*}(u), \lambda_{x}(\cdot)$ is continuous for each $x$. Now let $A$ be a nonempty index set, and choose $z \in X^{A}$ such that $u z=z$. Setting $Z=\mathcal{O}(z), Z$ is a minimal set, and $Z(u)$ carries its $\mathfrak{F}(u)$ topology. Because the uniformity of $X^{A}$ is generated by the uniformities of its factors $(X)$, it follows that a net $z_{\nu}$ in $Z(u)$ is $\mathfrak{F}(u)$ convergent if and only if it is $\mathfrak{F}(u)$ convergent in each coordinate. Therefore, if we define $\lambda_{z}(\gamma)=\gamma z, \gamma \in K(u), \lambda_{z}(\cdot)$ is continuous from $(K(u)$, $\left.\mathfrak{F}^{*}(u)\right)$ to $(Z(u), \mathfrak{F}(u))$. If we set $A=X(u)$ and take $z$ with $z(x)=x, x$ $\in X(u)$, and if $I \subseteq E(\mathfrak{X})$, so that $K(u)$ is effective, then $\lambda_{z}(\cdot)$ is one-to-one. However, because $\mathfrak{F}(u)$ is not $T_{2}$, we cannot infer immediately that $\lambda_{z}$ is a homeomorphism. In fact, it is, as will be seen later.

REMARK. Let $I \subseteq S$ or $E(\mathfrak{X})$ and let $\mathfrak{X} \rightarrow^{\pi} \mathscr{O}$ be a homomorphism of minimal flows. By equivariance and continuity, $X(u) \rightarrow^{\pi} Y(u)$ is onto and equivariant with respect to $K(u)$. (Use $\pi u$ and $\pi K(u)$ if $I \subseteq E(\mathscr{X})$.) As pseudometrics on $Y$ pull back to pseudometrics on $X, \pi$ is $\mathfrak{F}(u)$ continuous and $\mathfrak{F}^{*}(u)$ continuous. I shall later prove $\pi$ is $\mathfrak{F}(u)$ closed, which is not obvious.

I shall now describe the Ellis topology [34]. Fix a minimal ideal $I \subseteq S(G)$ or $E(\mathscr{X}), u \in J(I)$, and identify $g \in G$ with $s_{g} \in S(G)$ or $E(\mathfrak{X})$. If $V \subseteq G$ is a set such that $u \in(\bar{V})^{0}$, define $h(V)=\bar{V} \cap I$, so that $u \in h(V)^{0}$ (relative topology). As $I$ is a minimal set, Proposition 2.2 .2 implies the set $V u$ $=\left\{g \in G \mid g u \in h(V)^{0}\right\}$ is open and l.r.d. If $p \in h(V u)$, then $p=\lim _{\nu} g_{\nu}$ for some net in $V u$, and because $p u=p$ for any $p \in I, \lim _{\nu} g_{\nu} u=p$ also. By definition of $V u, g_{\nu} u \in h(V)^{0} \subseteq h(V)$, and therefore $p \in h(V)$. That is, $h(V u) \subseteq h(V)$. On the other hand, if $p \in h(V)^{0}$, choose a net $g_{\nu}$ in $V$ with $\lim _{\nu} g_{\nu}=p$. Again $g_{\nu} u \rightarrow p$, and therefore $g_{\nu} u \in h(V)^{0}$ for large $\nu$. Therefore, $g_{\nu} \in V u$ for large $\nu$, and so $p \in h(V u)$. The inclusions $h(V)^{0} \subseteq h(V u) \subseteq h(V)$ imply $h(V)^{0}=h(V u)^{0}$, and therefore

\subsubsection{Proposition (Ellis [34]). If $u \in \bar{V}^{0}$, then $(V u) u=V u$.}

REMARK. The inclusion $h(V u) \subseteq h(V)$, plus the proposition, imply the sets $\left\{h(V) \mid u \in(\bar{V})^{0}, V u=V\right\}$ form a base for the neighborhoods of $u$ in $I$.

Let $\mathcal{X}$ be a minimal flow, and fix $u \in J, x \in X(u)$. If $U$ is a neighborhood of $x$ in $X$ and $V \subseteq G$ an open set such that $V u=V$, define

$$
[U, V]_{u}=\bigcup_{g \in V} g^{-1} U \cap X(u) .
$$


I shall demonstrate that these sets comprise a basis for $\mathfrak{F}(u)$. As they also comprise a basis for the topology defined by Ellis [34], the two must coincide.

Let $x \in X(u), \sigma \in \Sigma, \varepsilon>0$ be given. Choose a neighborhood $U$ of $x$ such that $\sigma(x, z)<\varepsilon, z \in U$, and use the fact $u x=x$, plus the remark following Proposition 2.5.3 to find an open set $V$ such that $V u=V$ and $V x \subseteq U$. If $g \in V$ and $z \in g^{-1} U \cap X(u)$, then $\sigma(g x, g z) \leqslant \sigma(g x, x)+\sigma(x, g z)<2 \varepsilon$. Thus, $z \in U_{2 \varepsilon, \sigma}^{u}(x)$, and as $z$ is arbitrary, $[U, V]_{u} \subseteq U_{2 \varepsilon, \sigma}^{u}(x)$. Conversely, let $[U, V]_{u}$ be given containing $x \in U \cap X(u)$. There exists $\sigma \in \Sigma$ and $\varepsilon>0$ such that $\{z \mid \sigma(x, z)<\varepsilon\} \subseteq U$, and there exists $V_{0} \subseteq V$ such that $V_{0} u=V_{0}$ and $\sigma(x, z)<\varepsilon / 2, z \in V_{0} x . V_{0}$ is 1.r.d., and therefore there exist $g_{1}, \ldots, g_{k} \in G$ with $G=\cup_{j=1}^{k} g_{j}^{-1} V_{0}$. Choose $\sigma^{\prime} \in \Sigma$ and $\varepsilon^{\prime}>0$ so that if $\sigma^{\prime}\left(x^{\prime}, x^{\prime \prime}\right)<\varepsilon^{\prime}$, then $\sigma\left(g_{j} x^{\prime}, g_{j} x^{\prime \prime}\right)<\varepsilon / 2,1 \leqslant j \leqslant k$. I will now prove $U_{\varepsilon^{\prime}, \sigma^{\prime}}^{u}(x) \subseteq[U, V]_{u}$. To this end fix $z \in U_{\varepsilon^{\prime}, \sigma^{\prime}}^{u}(x)$, and choose $g \in G$ with $\sigma^{\prime}(g x, g z)<\varepsilon^{\prime}$. There exists $j$ with $g_{j} g \in V_{0}$, and by choice of $\sigma^{\prime}, \varepsilon^{\prime}, \sigma\left(g_{j} g x, g_{j} g z\right)<\varepsilon / 2$. Therefore,

$$
\sigma\left(x, g_{j} g z\right) \leqslant \sigma\left(x, g_{j} g x\right)+\sigma\left(g_{j} g x, g_{j} g z\right)<\varepsilon / 2+\varepsilon / 2=\varepsilon .
$$

But this implies $g_{j} g z \in U$ or $z \in\left(g_{j} g\right)^{-1} U \subseteq\left[U, V_{0}\right]_{u} \subseteq[U, V]_{u}$. Thus, $U_{\varepsilon^{\prime}, \sigma^{\prime}}^{u}(x) \subseteq[U, V]_{u}$, as claimed. We have

2.5.5. PROPOSITION (SEE ALSO[40]). The sets $[U, V]_{u}$ of (2.5.4) form a basis for $\Re(u)$.

The proposition leads to the elegant characterization of $\mathfrak{F}(u)$ closures due to Ellis, Glasner and Shapiro [40]. Below, topological entities are understood to be with respect to the "regular" topologies unless $\mathfrak{F}(u), \mathfrak{F}^{*}(u)$ are specified. It is convenient here to assume $I \subseteq S$, so that $u \circ A$ is defined.

2.5.6. Proposition (Ellis, Glasner and Shapiro [40]). If $A \subseteq X(u)$, then $\operatorname{cls}_{\mathfrak{T}(u)} A=u \circ A \cap X(u)=u(u \circ A)$. (Recall that $u \circ A=u \circ \bar{A}$.)

Proof. $(u \circ A) \cap X(u) \subseteq u(u \circ A) \subseteq u \circ(u \circ A)=u \circ A$. Therefore, $u$ $\circ A \cap X(u)=u(u \circ A)$. A point $x \in X(u)$ belongs to $\operatorname{cls}_{\widetilde{T}(u)} A$ if and only if $[U, V]_{u} \cap A \neq \varnothing$ whenever $U$ is a neighborhood of $x$ and $V u=u$. This, in turn, is if and only if there exist nets $x_{\nu}$ in $A, g_{\nu}$ in $G$ such that $g_{\nu} \rightarrow u$ and $g_{\nu} x_{\nu} \rightarrow x$. That is, if and only if $x \in u \circ A$ and $u x=x$.

2.5.7. Proposition. Let $\mathcal{X} \rightarrow^{\pi}$ Oु be a homomorphism of minimal flows. Then $X(u) \rightarrow^{\pi} Y(u)$ is an onto $\mathfrak{F}(u)$-continuous and $\mathfrak{F}(u)$-closed map.

Proof. We have only to prove $\pi A$ is $\mathfrak{F}(u)$ closed in $Y(u)$ if $A$ is $\mathfrak{F}(u)$ closed in $X(u)$. It is easily checked that $\pi(u \circ A)=u \circ \pi A$, and therefore if $u(u \circ \pi A)$ $\neq \pi A$, there exists $x \in u \circ A$ with $\pi x \in u(u \circ \pi A)$ but not in $\pi A$. However, $u x \in u(u \circ A) \subseteq u \circ A$ also, and as $A=u(u \circ A)$ because $A$ is $\mathfrak{F}(u)$ closed, $\pi x=\pi u x \in \pi A$. Thus, $\pi A$ is $\mathfrak{i}(u)$ closed.

2.5.8. Corollary [40]. Let $\mathfrak{X} \rightarrow^{\pi}$ OY be a homomorphism of minimal flows, and suppose $\pi$ is one-to-one on $X(u)$. Then $\pi$ is an $\mathfrak{F}(u)$ homeomorphism.

REMARK. If $\pi$ is one-to-one on $X(u)$ for one $u$ it is for all $u$ (since $X(u) \rightarrow^{v} X(v)$ is one-to-one for $\left.v \in J\right)$. This implies $\pi$ is a proximal homomorphism. Conversely, if $\pi$ is proximal, $\pi$ is one-to-one on $X(u)$ for all $u$. 
If $I \subseteq E(\mathfrak{X}), u \in J(I)$, then $I(u)=u I(=K(u))$ carries its own $\mathfrak{F}(u)$ topology, defined using the flow $g=(G, I)$. We shall prove $(I(u)$, $F(u))$ and $\left(K(u), \mathfrak{F}^{*}(u)\right)$ are homeomorphic. First suppose $\lim _{\nu} p_{\nu}=p(\mathfrak{F}(u))$. For each $x \in X(u), g \rightarrow^{\lambda x} \mathcal{X}$ is a homomorphism of flows, and therefore

$$
\mathfrak{F}(u) \lim _{\nu} p_{\nu} x=\mathfrak{F}(u) \lim \lambda_{x}\left(p_{\nu}\right)=\lambda_{x}(p)=p x .
$$

Since $x$ is arbitrary, the definition of $\mathfrak{F}^{*}(u)$ implies $\mathscr{F}^{*}(u) \lim _{\nu} p_{\nu}=p$, and the injection $(I(u), \mathfrak{F}(u)) \rightarrow^{i}\left(K(u), \mathfrak{F}^{*}(u)\right)$ is continuous. For the reverse map, set up $X^{X(u)}$ and $z$ with $z(x)=x, x \in X(u)$, as before. If $Z=\mathfrak{O}(z), Z(u)=u Z$, there is a commutative diagram $\left(\lambda_{z}^{0}(\gamma)=\gamma z\right)$

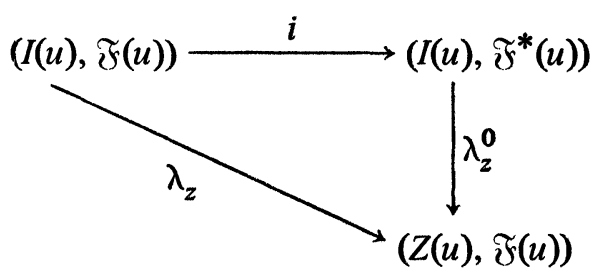

in which all maps are one-to-one and known to be continuous. As $\lambda_{z}$ is also closed, $i^{-1}$ is also continuous. We have

2.5.9. Proposition. Let $\mathfrak{X}$ be a minimal flow, and let $I \subseteq E(\mathfrak{X})$ be a minimal ideal. For each $u \in J(I),(I(u), \mathfrak{F}(u))$ and $\left(I(u), \mathfrak{F}^{*}(u)\right)$ are the same. In particular, $(I(u), \mathfrak{F}(u))$ is an F-group.

2.6. The relativized equicontinuous structure relation. Recall that if $\mathscr{X} \rightarrow \pi$ ơ is a homomorphism of minimal flows, then $E_{\pi}$ is the "relativized equicontinuous structure relation," the least closed invariant equivalence relation containing $Q_{\pi}$. The theorem to follow is new and will be the principal result of this section. $I$ is a minimal ideal in $S$ (for now) and $u \in J$ a fixed idempotent.

2.6.1. THEOREM. Let $\mathcal{X} \rightarrow^{\pi}$ Oु be a homomorphism of minimal flows which is an $R I C$ extension. If $x \in X(u)$, then for every relative $\mathfrak{F}(u)$ neighborhood $\alpha$ of $x$ in $u \pi^{-1} \pi x\left(=X(u) \cap \pi^{-1} \pi x\right)$, we have $E_{\pi}[x] \subseteq u \circ \alpha$.

One of the consequences of Theorem 2.6.1 is the following result which has been obtained independently and earlier by Ellis (oral communication):

2.6.2. THEOREM. Let $\mathfrak{X} \rightarrow^{\pi}$ oy be a homomorphism of minimal flows which satisfies the Bronstein condition (Definition 2.3.3). Then $Q_{\pi}=E_{\pi}$; i.e., $Q_{\pi}$ is already an equivalence relation.

If $\mathfrak{X} \rightarrow^{\pi} \mathcal{Y}, \mathscr{Z} \rightarrow^{\lambda} \mathcal{Y}$ are homomorphisms, define $\mathfrak{R}_{\pi, \lambda}=\left(G, R_{\pi, \lambda}\right)$, where $R_{\pi, \lambda}=\{(x, z) \mid \pi x=\lambda z\} .\left(\Re_{\pi, \pi}=\Re_{\pi}.\right)$ (Similarly, given $\mathfrak{X}_{j} \rightarrow_{\pi j} \mathcal{Q}_{j} j=1$, $\ldots, n$, define $\Re_{\pi_{1} \pi_{2}} \ldots \pi_{n}$.) I shall say $(\pi, \lambda)$ (resp. $\left(\pi_{1}, \ldots, \pi_{n}\right)$ ) satisfies the generalized Bronstein condition (g.B.c.) if the a.p. points are dense in $R_{\pi, \lambda}$ (resp. $\left.R_{\pi_{1}, \ldots, \pi_{n}}\right)$. Another consequence of Theorem 2.6.1 will be

2.6.3. TheOREM. Let $\mathfrak{X} \rightarrow{ }^{\pi}$ y be a homomorphism of minimal flows which satisfies the Bronstein condition, and suppose that $E_{\pi}=R_{\pi}$. If $\mathscr{Z} \rightarrow^{\lambda} \mathscr{Y}$ is a homomorphism of flows such that $\mathscr{Z}$ is topologically transitive and if $(\pi, \lambda)$ satisfies 
the generalized Bronstein condition, then $\Re_{\pi, \lambda}$ is topologically transitive.

REMARK. If $\mathscr{X} \rightarrow^{\pi} \mathscr{Y}$ is an RIC extension, and if $\mathscr{Z} \rightarrow^{\lambda} \mathscr{Y}$ is such that $\mathscr{Z}$ contains a dense set of a.p. points, then I claim $(\pi, \lambda)$ satisfies the g.B.c. To see this, let $w=(x, z) \in R_{\pi, \lambda}$, and let $W$ be a neighborhood of $w$. The projection of $W$ onto the first coordinate is a neighborhood of $x$ which under $\pi$ is sent to a neighborhood $U$ of $\pi x=y=\lambda z \cdot \lambda^{-1} U$ is a neighborhood of $z$ and we may choose $z^{\prime} \in \lambda^{-1} U$ such that $z^{\prime}$ is an a.p. point and $\left(x^{\prime}, z^{\prime}\right) \in W$ for some $x^{\prime} \in X$. Suppose $u z^{\prime}=z^{\prime}$, and note that $u \circ\left(u \pi^{-1} \pi x^{\prime}\right)=\pi^{-1} \pi x^{\prime}$ because $\pi$ is RIC. There exist nets $g_{\nu}$ in $G$ and $x_{\nu}^{\prime}$ in $u \pi^{-1} \pi x^{\prime}$ such that $g_{v} \rightarrow u$ and $g_{\nu} x_{v}^{\prime} \rightarrow x^{\prime}$. Then $\lim _{\nu} g_{\nu}\left(x_{\nu}^{\prime}, z^{\prime}\right)=\left(x^{\prime}, z^{\prime}\right)$. As $g_{\nu}\left(x_{\nu}^{\prime}, z^{\prime}\right)$ is an a.p. point for each $\boldsymbol{\nu}$, our claim is established. Therefore, in the special case $\mathcal{Y}=\Omega$ ( $\mathfrak{X}$ incontractible), Theorem 2.6.3 implies Theorem 2.1.6. A simple induction based on Theorem 2.6.3 implies the following, which in the metrizable case is due to McMahon [88].

2.6.4. THEOREM. Let $\mathfrak{X} \rightarrow^{\pi}$ Oy be a homomorphism of minimal flows, and suppose for each $n \geqslant 2$ that $(\pi, \pi, \ldots, \pi)$ ( $n$ times) satisfies the g.B.c. Then $\Re_{(\pi, \pi, \ldots, \pi)}$ is topologically transitive.

In connection with Theorem 2.6.2, Ellis proved [38] that the Bronstein condition implies $E_{\pi}=Q_{\pi} P_{\pi}$. "Unrelativized" versions $(\mathcal{Y}=\Omega)$ of the theorem were proved in special cases by Peterson ([105]; $G$ abelian) and Ellis and Keynes ([42]; $X(u)$ contains an orbit). A different characterization of the (unrelativized) equicontinuous structure theorem is given in [150] ( $G$ abelian) and [143] ( $G$ amenable) and can be used to identify it with the regionally proximal relation (cf. [105]).

In what follows I shall combine the approaches of [50], [146] and [38], [40].

Let $\mathcal{X} \rightarrow^{\pi} \mathcal{Y}$ be a homomorphism of minimal flows, fix $y \in Y(u)$, and set $W=X(u) \cap \pi^{-1} y . W$ is $\mathfrak{F}(u)$ closed (because $\{y\}$ is) and therefore compact. Set $\Gamma=\left\{\gamma \in K(u) \mid \gamma \pi^{-1} y \subseteq \pi^{-1} y\right\}$. Then $\Gamma$ is an $\mathfrak{F}(u)\left(=\mathfrak{F}^{*}(u)\right)$ closed subgroup of $K(u)$, hence an $F$-group. Also, $\Gamma$ is transitive on $W$ (but possibly not effective).

If $x \in W$ we use $N_{x}$ to denote the set of (relative) $\mathfrak{F}(u)$ neighborhoods of $x$ in $W$. Define $E(x)=\cap_{U \in N_{x}} \operatorname{cls}_{\mathfrak{\mho}(u)} U$ and $\Gamma(x)=\{\gamma \in \Gamma \mid \gamma x \in E(x)\}$. Both $E(x)$ and $\Gamma(x)$ are $\mathfrak{F}(u)$ closed, and for all $x \in W, \gamma \in \Gamma$ we have

$$
\Gamma(\gamma x)=\gamma \Gamma(x) \gamma^{-1} .
$$

$\Gamma(x)$ is trivially expressed as

$$
\Gamma(x)=\bigcap_{U \in N_{x}}\left\{\gamma \in \Gamma \mid \gamma x \in \operatorname{cls}_{\mathfrak{r}(u)} U\right\}
$$

and clearly contains

$$
\Gamma^{\prime}(x)=\bigcap_{U \in N_{x}} \operatorname{cls}_{\mathfrak{r}(u)}\{\gamma \in \Gamma \mid \gamma x \in U\} .
$$

However, because $\Gamma$ is transitive and the map $I(u) \rightarrow^{\lambda_{x}} X(u)$ given by $\lambda_{x}(p)=p x$ is $\mathfrak{F}(u)$ closed, we have for each $U \in N_{x}$ that $\operatorname{cls}_{\mathfrak{s}(u)}\{\gamma \in \Gamma \mid$ $\gamma x \in U\}=\left\{\gamma \in \Gamma \mid \gamma x \in \operatorname{cls}_{\mathfrak{F}(u)} U\right\}$. That is, $\Gamma^{\prime}(x)=\Gamma(x)$. It is not difficult 
to see that $\Gamma^{\prime}(x)$ is a group, and therefore $\Gamma(x)$ is a group (Ellis [38]). Now (2.6.5) implies $E(x)=E\left(x^{\prime}\right)$ if $x^{\prime} \in E(x)$, or even if $E(x) \cap E\left(x^{\prime}\right) \neq \varnothing$. Therefore, the relation $x E x^{\prime}$ if $x^{\prime} \in E(x)$ is an equivalence relation. By (2.6.5), $E(\gamma x)=\gamma E(x), \gamma \in \Gamma$. In fact, if $\gamma \in I$ is arbitrary, and if $v \in J$ is such that $v \gamma=\gamma$, then $E(\gamma x)=\gamma E(x)$ in the sense that if $E(\gamma x)$ is formed with respect to $(X(v), \mathfrak{F}(v))$ and $v M$, then $E(\gamma x)=\gamma E(x)$. This is because the map $(X(u), \mathfrak{F}(u)) \rightarrow^{v}(X(v), \mathfrak{F}(v))$ is, by (2.5.1), a homeomorphism.

Now let $L=W / E$ with the $\mathfrak{F}(u)$ quotient topology, and denote the canonical map by $W \rightarrow^{\mu} L$. For any $x \in W$ the map $\eta_{x}=\mu \lambda_{x}$ induces a continuous map from $\Gamma / \Gamma(x)$ with the quotient $(\mathfrak{F}(u))$ topology to $W$. Moreover, this map is one-to-one. Now let $x, x^{\prime} \in W$ be such that $\mu x \neq \mu x^{\prime}$. There exist $U \in N_{x}, U^{\prime} \in N_{x^{\prime}}$ such that $\operatorname{cls}_{\mathfrak{i}(u)} U \cap \operatorname{cls}_{\mathfrak{i}(u)} U^{\prime}=\varnothing$. Define $\Gamma_{A}\left(x^{\prime \prime}\right)=\left\{\gamma \in \Gamma \mid \gamma x^{\prime \prime} \in A\right\}$. I shall prove $\Gamma_{U}(x) \Gamma(x) \cap \Gamma_{U^{\prime}}\left(x^{\prime}\right) \Gamma\left(x^{\prime}\right)=\varnothing$, which implies $\Gamma / \Gamma(x)$ is $T_{2}$. Therefore, $L$ is $T_{2}$ and $\Gamma / \Gamma(x), L$ are homeomorphic. Now by (2.6.6)-(2.6.6') we have for any $\gamma \in \Gamma_{U}(x)$ that $\Gamma(\gamma x)$ $\subseteq \operatorname{cls}_{\mathfrak{F}(u)} \Gamma_{U}(\gamma x)=\operatorname{cls}_{\mathfrak{r}(u)} \Gamma_{U}(x) \gamma^{-1}, \quad$ or, what is the same, $\gamma \Gamma(x)$ $\subseteq \operatorname{cls}_{\mathfrak{f}(u)} \Gamma_{U}(x)$. Let $U_{0}=\operatorname{cls}_{\mathfrak{F}(u)} U, U_{0}^{\prime}=\operatorname{cls}_{\mathfrak{r}(u)} U^{\prime}$, and note that, as observed earlier, $\operatorname{cls}_{\mathfrak{F}(u)} \Gamma_{U}(x)=\Gamma_{U_{0}}(x), \operatorname{cls}_{\mathfrak{F}(u)} \Gamma_{U^{\prime}}\left(x^{\prime}\right)=\Gamma_{U_{0}^{\prime}}\left(x^{\prime}\right)$. It follows that $\Gamma_{U}(x) \Gamma(x) \subseteq \Gamma_{U_{0}}(x)$ and $\Gamma_{U^{\prime}}(x) \Gamma(x) \subseteq \Gamma_{U_{0}^{\prime}}(x)$ are disjoint.

Next, define $\Gamma_{0}=\bigcap_{x \in W} \Gamma(x)=\bigcap_{\gamma \in \Gamma} \gamma \Gamma\left(x_{0}\right) \gamma^{-1}$ (any fixed $x_{0} \in W$ ). $\Gamma_{0}$ is a normal subgroup of $\Gamma$, and by arguments similar to the above $H=\Gamma / \Gamma_{0}$ is $T_{2}$. As $H$ is also an $F$ group, the separate continuity theorem implies $H$ is a topological group. Note also that because $\Gamma$ is $\mathfrak{F}(u)$ compact, the canonical map $\Gamma \rightarrow^{\lambda} H$ is closed. Now from the triangle of natural maps

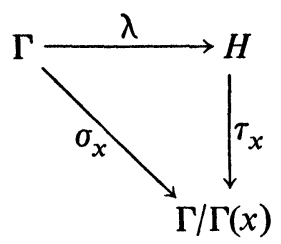

we infer that $\tau_{x}$ is continuous because $\sigma_{x}$ is continuous and $\lambda$ is closed. It follows that $L$ is a homogeneous space of $H$.

Let $\delta_{0}$ be a continuous $H$-invariant pseudometric on $L$. Lift $\delta_{0}$ to an $\mathfrak{F}(u)$ continuous, $\Gamma$ invariant pseudometric on $W$, retaining the notation $\delta_{0}$. Note that if $\left(x_{1}, x_{2}\right)=z \notin E$, then $\delta_{0}$ can be chosen with $\delta_{0}(z)>0$. For any $z \in W \times W, g \in G$, such that $g z \in W \times W$ we have $u g u \in \Gamma, u g u z=g z$, and therefore $\delta_{0}(g z)=\delta_{0}(u g u z)=\delta_{0}(z)$. It follows that $\delta_{0}(g z)=\delta_{0}(z), g$ $\in G, z \in W \times W$ defines $\delta_{0}$ on $R_{\pi}^{\prime}(u)=G(W \times W) \subseteq R_{\pi}$. The following lemma is elementary [50], [146].

2.6.7. Lemma. For every $\varepsilon>0$ there exist $\sigma \in \Sigma$ and $\varepsilon^{\prime}>0$ such that if $z \in R_{\pi}^{\prime}(u)$ and $F_{\sigma}(z)<\varepsilon^{\prime}$, then $\delta_{0}(z)<\varepsilon$.

I now make the assumption that $\mathcal{X}$ is an RIC extension of $\mathcal{O}$, which implies $R_{\pi}=\overline{R_{\pi}^{\prime}(u)}$. Let $I \subseteq S$ be a minimal ideal $(u \in I$ now and $u y=y)$, and recall that if $p \in v I, v \in J$, then $p \circ C(y, u)=C(p y, v)$. If $z \in R_{\pi}, z=\left(x_{1}\right.$, $\left.x_{2}\right)$, and if $\pi x_{1}=y^{\prime}$, let $I_{y^{\prime}}=\left\{p \in I \mid p y=y^{\prime}\right\}$, and define 


$$
\delta(z)=\sup _{p \in I_{y^{\prime}}} \sup _{\substack{g_{p} \rightarrow p ; z_{\nu} z_{\nu} \rightarrow z \\ z_{\nu} \in W \times W}} \delta_{0}\left(z_{\nu}\right),
$$

noting that $\delta$ is u.s.c., invariant, and $\delta(z) \geqslant \delta_{0}(z), z \in R_{\pi}^{\prime}(u)$. In particular, if $z \in W \times W$ does not belong to $E$, there exists $\delta_{0}$ such that $\delta(z)>0$.

2.6.9. LeMMA (COMPARE WITH [50], [146]). With notations as above, $\delta(\cdot)$ defines a pseudometric on $\pi^{-1} y^{\prime}, y^{\prime} \in Y$.

Proof. Let $x_{1}, x_{2}, x_{3} \in \pi^{-1} y^{\prime}$, and set $z=\left(x_{1}, x_{3}\right), z^{\prime}=\left(x_{1}, x_{2}\right), z^{\prime \prime}$ $=\left(x_{2}, x_{3}\right)$. Given $\varepsilon>0$ there exist $p \in I, p y=y^{\prime}$, and nets $g_{v},\left(x_{1}^{\nu}, x_{3}^{\nu}\right)$ $\in W \times W$, such that $g_{\nu} \rightarrow p, g_{\nu}\left(x_{1}^{\nu}, x_{3}^{\nu}\right) \rightarrow\left(x_{1}, x_{3}\right)$, and $\liminf _{v} \delta_{0}\left(g_{\nu}\left(x_{1}^{\nu}, x_{3}^{\nu}\right)\right)$ $\geqslant \delta(z)-\varepsilon$. In the topology of $2^{X}, g_{\nu} \bar{W} \rightarrow C\left(y^{\prime}, v\right)(v p=p)$. Therefore, after passing to a subnet, if necessary, we may suppose $x_{2}^{\nu} \in W$ and $g_{\nu} x_{2}^{\nu} \rightarrow x_{2}$. Set $z_{\nu}=\left(x_{1}^{\nu}, x_{3}^{\nu}\right), z_{\nu}^{\prime}=\left(x_{1}^{\nu}, x_{2}^{\nu}\right), z_{\nu}^{\prime \prime}=\left(x_{2}^{\nu}, x_{3}^{\nu}\right)$. Then

$$
\begin{aligned}
\delta(z) & \leqslant \varepsilon+\lim _{\nu} \inf \delta_{0}\left(g_{\nu} z_{\nu}\right) \leqslant \varepsilon+\limsup _{\nu} \delta_{0}\left(g_{\nu} z_{\nu}\right) \\
& \leqslant \varepsilon+\lim \sup \left\{\delta_{0}\left(g_{\nu} z_{\nu}^{\prime}\right)+\delta_{0}\left(g_{\nu} z_{\nu}^{\prime \prime}\right)\right\} \\
& \leqslant \varepsilon+\delta\left(z^{\prime}\right)+\delta\left(z^{\prime \prime}\right) .
\end{aligned}
$$

Letting $\varepsilon \rightarrow 0$, our claim is established.

An immediate consequence of Lemma 2.6.7 and the fact that $F_{\sigma}$ is u.s.c. is

2.6.7'. Lemma. For every $\varepsilon>0$ there exist $\sigma \in \Sigma$ and $\varepsilon^{\prime}>0$ (the same as in Lemma 2.6.7) such that if $z \in R_{\pi}$ and $F_{\sigma}(z)<\varepsilon^{\prime}$, then $\delta(z)<\varepsilon$.

Now define $\Delta_{c}, c>0$, by

$$
\Delta_{c}=\left\{z \in R_{\pi} \mid \liminf _{\substack{z^{\prime} \rightarrow z \\ z^{\prime} \in R_{\pi}}} \delta\left(z^{\prime}\right) \leqslant \delta(z)-c\right\} .
$$

Because $\delta$ is u.s.c. and invariant, $\Delta_{c}$ is closed and invariant. Moreover, if $\Delta_{c}=\varnothing, c>0$, then $\delta$ is continuous. I shall prove $\Delta_{c}=\varnothing$ by contradiction, in a fashion similar to that in [50], [146].

If $\Delta_{c} \neq \varnothing$, there is a minimal set $Z \subseteq \Delta_{c}$. Let $\mathcal{Z}^{\mu} \rightarrow^{\mu}$ oX be the map onto the first coordinate, and form the sets $C_{\mu}(x, v), x \in X, v \in J, v x=x$. Of course, $C_{\mu}(x, v) \subseteq\{x\} x \pi^{-1} \pi x \subseteq R_{\pi}$.

If we restrict $z, z^{\prime}$ in (2.6.10) to lie in $Z$, we obtain a closed invariant set $\Delta_{c}^{\prime} \subseteq Z$. As $\mathscr{Z}$ is minimal, either $\Delta_{c}^{\prime}=\varnothing$ or $\Delta_{c}^{\prime}=Z$. If $\Delta_{c}^{\prime}=Z,\left.\delta\right|_{Z}$ has no point of continuity. However, being u.s.c., $\left.\delta\right|_{Z}$ has a point of continuity.

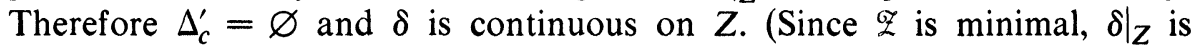
constant.)

Now fix $z \in Z$ and use the definition of $\Delta_{c}$ to find a net $z_{\nu} \rightarrow z$ in $R_{\pi}$ such that $\delta\left(z_{\nu}\right) \leqslant \delta(z)-3 c / 4$. Write $z=\left(x_{1}, x_{2}\right), z_{\nu}=\left(x_{1}^{\nu}, x_{2}^{\nu}\right)$, and choose $u, u_{\nu}$ in $J$ with $u z=z\left(z\right.$ is an a.p. point!) and $u_{\nu} x_{1}^{\nu}=x_{1}^{\nu}$. Matters may be arranged so that $\lim _{\nu} C_{\mu}\left(x_{1}^{\nu}, u_{v}\right)=C_{\mu}\left(x_{1}, v\right)$ exists. Possibly $v \neq u$, but at least $v x_{1}$ $=x_{1}$. Recall that $u \circ C_{\mu}(x, v)=C_{\mu}\left(u x_{1}, u v\right)=C_{\mu}\left(x_{1}, u\right)$. Choose a net $g_{\tau}$ in $G$ such that $g_{\tau} \rightarrow u$. Of course, $g_{\tau} z \rightarrow z$, and $g_{\tau} C_{\mu}\left(x_{1}, v\right) \rightarrow C_{\mu}\left(x_{1}, u\right)\left(\right.$ in $\left.2^{X}\right)$.

If $\alpha \in \mathcal{Q}$ (the uniformity for $X$ ), choose $g_{\tau}$ so that 
(i) $\left(x_{j}, g_{\tau} x_{j}\right) \in \alpha, j=1,2$, and

(ii) $\left(g_{\tau} C_{\mu}\left(x_{1}, v\right), C_{\mu}\left(x_{1}, u\right)\right) \in \alpha^{*}(\S 2.3)$.

Denote the dependence on $\alpha$ by $h_{\alpha}=g_{\tau}$. Use the continuity of $h_{\alpha}$ to find $\nu$ such that

(i') $\left(x_{j}^{\nu}, h_{\alpha} x_{j}^{\nu}\right) \in \alpha$, and

(ii') $\left(h_{\alpha} C_{\mu}\left(x_{1}^{\nu}, u_{v}\right), C_{\mu}\left(x_{1}, u\right)\right) \in \alpha^{*}$.

Set $\left(w_{1}^{\alpha}, w_{2}^{\alpha}\right)=\left(x_{1}^{\nu}, x_{2}^{\nu}\right)$, and pick $\bar{w}_{2}^{\alpha} \in C_{\mu}\left(x_{1}^{\nu}, u_{\nu}\right)$ such that $\left(x_{2}, h_{\alpha} \bar{w}_{2}^{\alpha}\right) \in \alpha$. Now $\lim _{\alpha} h_{\alpha}\left(w_{1}^{\alpha}, \bar{w}_{2}^{\alpha}\right)=z$, and $\left(w_{1}^{\alpha}, \bar{w}_{2}^{\alpha}\right) \in Z$. Since $\lim _{\alpha} h_{\alpha}\left(w_{2}^{\alpha}, \bar{w}_{2}^{\alpha}\right)=\left(x_{2}, x_{2}\right)$ $\in \Delta$, we have from Lemmas 2.6 .9 and 2.6.7':

$$
\limsup _{\alpha}\left|\delta\left(h_{\alpha}\left(w_{1}^{\alpha}, w_{2}^{\alpha}\right)\right)-\delta\left(h_{\alpha}\left(w_{1}^{\alpha}, \bar{w}_{2}^{\alpha}\right)\right)\right| \leqslant \limsup _{\alpha} \delta\left(h_{\alpha}\left(w_{2}^{\alpha}, \bar{w}_{2}^{\alpha}\right)\right)=0 .
$$

But this contradicts the fact that $\delta\left(h_{\alpha}\left(w_{1}^{\alpha}, \bar{w}_{2}^{\alpha}\right)\right)=\delta(z)$ and $\delta\left(w_{1}^{\alpha}, w_{2}^{\alpha}\right)=\delta\left(z_{\nu}\right)$ $\leqslant \delta(z)-3 c / 4$. Thus, $\Delta_{c}=\varnothing$, and $\delta$ is continuous.

Now return to the general (non-RIC) case, and define $R_{\pi}(u)=\overline{R_{\pi}^{\prime}(u)}$. As essentially noted in $\$ 2.3, R_{\pi}(u)$ is the closure of the a.p. points in $R_{\pi}$. If $X^{\prime} \rightarrow \pi^{\prime} \mathscr{Y}^{\prime}$ is as attached in $\left(2.3 .1^{\prime}\right)$, then by the argument for Lemma 2.3.4, $\tau \times \tau R_{\pi^{\prime}}=R_{\pi}(u)$ (= $R_{\pi}$ if $\pi$ satisfies the Bronstein condition). Under $\tau, X(u)$ and $X^{\prime}(u)$ are $\mathfrak{F}(u)$ homeomorphic (Corollary 2.5.8) and, therefore, $W, \Gamma, L$, etc. may be formed using $\pi^{\prime}$ instead of $\pi$ with the same result. Let $\delta$ be as constructed above, except on $R_{\pi^{\prime}}$. If $c>0$ the set $\Delta_{c}$ of $z \in R_{\pi}(u)$ such that there exist $z_{1}, z_{2}, \tau \times \tau\left(z_{j}\right)=z$ and $\left|\delta\left(z_{1}\right)-\delta\left(z_{2}\right)\right| \geqslant c$ is closed and invariant, and, therefore, if nonempty, contains an a.p. point. However, if $z=\left(x_{1}, x_{2}\right)$ $\in R_{\pi}(u)$ is an a.p. point, say $u z=z$, there exists $z^{\prime}=\left(x_{1}^{\prime}, x_{2}^{\prime}\right) \in R_{\pi^{\prime}}, u z^{\prime}$ $=z^{\prime}$ with $\tau \times \tau z^{\prime}=z$. If $z^{\prime \prime}=\left(x_{1}^{\prime \prime}, x_{2}^{\prime \prime}\right) \in R_{\pi^{\prime}}$ satisfies $\tau \times \tau z^{\prime \prime}=z$, then because $\tau$ is proximal, $u x_{1}^{\prime \prime}=x_{1}^{\prime}$ and $u x_{2}^{\prime \prime}=x_{2}^{\prime}$. Thus, $\delta\left(z^{\prime}\right)=\delta\left(u z^{\prime \prime}\right)$ $=\delta\left(z^{\prime \prime}\right)$, meaning $z \notin \Delta_{c}$. Thus, $\Delta_{c}=\varnothing$, and $\delta$ may be regarded as an element of $C\left(R_{\pi}(u)\right)$. As $C\left(y^{\prime}, u^{\prime}\right)$ is homeomorphic under $\tau$ to some fiber of $\mathfrak{X}^{\prime} \rightarrow^{\pi^{\prime}} \mathscr{Y}^{\prime}$, it follows that $\delta$ defines a pseudometric on $C\left(y^{\prime}, u^{\prime}\right)$ for all $y^{\prime} \in Y, u^{\prime} \in J, u^{\prime} y^{\prime}=y^{\prime}$. In particular, if $\pi$ satisfies the Bronstein condition, and if $x_{1}, x_{2}, x_{3} \in \pi^{-1} y(u y=y)$, then

$$
\begin{aligned}
\delta\left(x_{1}, x_{3}\right) & =\delta\left(u x_{1}, u x_{3}\right) \leqslant \delta\left(u x_{1}, u x_{2}\right)+\delta\left(u x_{2}, u x_{3}\right) \\
& =\delta\left(x_{1}, x_{2}\right)+\delta\left(x_{2}, x_{3}\right) .
\end{aligned}
$$

That is, $\delta$ defines a pseudometric on $\pi^{-1} y$. It follows that $\delta(z)=0$ defines an invariant closed equivalence relation.

Denote by $\Sigma_{\pi}$ the set of functions $\delta$ which arise by the above procedure. By the last paragraph, if $\pi$ satisfies the Bronstein condition, the set

$$
E_{\pi}^{\prime}=\left\{z \in R_{\pi} \mid \delta(z)=0, \text { all } \delta \in \Sigma_{\pi}\right\}
$$

is a closed, invariant equivalence relation. We note first that $Q_{\pi} \subseteq E_{\pi}^{\prime}$. For if $z \in Q_{\pi}$, there are nets $z_{v}$ in $R_{\pi}$ and $g_{\nu}$ in $G$ such that $z_{v} \rightarrow z$ and $g_{v} z_{v} \rightarrow w$ $\in \Delta$. If $\delta \in \Sigma_{\pi}$, then $\delta(z)=\lim _{\nu} \delta\left(z_{\nu}\right)=\lim _{\nu} \delta\left(g_{\nu} z_{\nu}\right)=\delta(w)=0$. Since $\delta$ is arbitrary, $z \in E_{\pi}^{\prime}$, and since $z \in Q_{\pi}$ is arbitrary, $Q_{\pi} \subseteq E_{\pi}^{\prime}$. By definition, therefore, $E_{\pi} \subseteq E_{\pi}^{\prime}$. Now let $\mathfrak{X}^{\prime} \rightarrow \pi^{\pi^{\prime}} \mathcal{Y}^{\prime}$ be as in (2.3.1'). The construction of $\delta \in \Sigma_{\pi}$ above makes it evident that $\tau \times \tau\left(E_{\pi^{\prime}}^{\prime}\right)=E_{\pi}^{\prime}$, and as $\tau \times \tau\left(Q_{\pi^{\prime}}\right)$ $\subseteq Q_{\pi}$, it will follow that $E_{\pi}^{\prime} \subseteq Q_{\pi} \subseteq E_{\pi}$ if we prove $Q_{\pi^{\prime}}=E_{\pi^{\prime}}^{\prime}$. In other 
words, Theorem 2.6.2 is true under the Bronstein condition if it is true for RIC extensions. In order to prove this, it is necessary first to prove Theorem 2.6.1.

Let $W, \Gamma, u$ be as in our earlier discussion, and fix $x \in W$. As before $N_{x}$ is the set of $\mathfrak{F}(u)$ neighborhoods of $x$ in $W$. Our goal is to prove $u \circ \alpha \supseteq E_{\pi}^{\prime}[x]$ for which purpose we may suppose $\alpha$ has the form $\alpha=W \cap U_{\varepsilon, \sigma}(x)$ for some $\varepsilon>0, \sigma \in \Sigma$. Define $\Gamma_{\alpha}=\Gamma_{\alpha}(x)=\{\gamma \in \Gamma \mid \gamma x \in \alpha\}$, so that $\Gamma_{\alpha}=\Gamma_{\alpha}^{-1}$. One proves readily that $u \circ \alpha=u \circ\left(\Gamma_{\alpha} x\right)=\left(u \circ \Gamma_{\alpha}\right) x$. Because $\left(u \circ \Gamma_{\alpha}\right) \cap \Gamma$ $=\operatorname{cls}_{\mathfrak{F}(u)} \Gamma_{\alpha}, u \circ \Gamma_{\alpha} \supseteq \Gamma(x)$. In fact, if $\gamma \in \Gamma_{\alpha}, \Gamma(\gamma x) \gamma \subseteq u \circ \Gamma_{\alpha}$, or what is the same by (2.6.5), $\gamma \Gamma(x) \subseteq u \circ \Gamma_{\alpha}$. That is, $\Gamma_{\alpha} \Gamma(x) \subseteq u \circ \Gamma_{\alpha}$, and applying $u$ once more $\left(u \circ \Gamma_{\alpha}\right) \Gamma(x) \subseteq u \circ \Gamma_{\alpha}$.

Suppose $\gamma \in\left(u \circ \Gamma_{\alpha}\right) \cap \Gamma$ is not an $\mathfrak{F}(u)$ interior point. There exists a net $\gamma_{\nu}$ in $\Gamma, \gamma_{\nu} \notin u \circ \Gamma_{\alpha}$, such that $\mathfrak{F}(u) \lim _{\nu} \gamma_{\nu}=\gamma$. For large $\nu$ it must be that $\gamma\left(\gamma \gamma_{v}^{-1}\right) \in \gamma \Gamma_{\alpha}$, or $\gamma \in \Gamma_{\alpha} \gamma_{\nu}$. Therefore, if $A$ denotes the $\mathfrak{F}(u)$ interior of $u \circ \Gamma_{\alpha}\left(\supseteq \Gamma_{\alpha}\right)$, the set $\left\{A, \Gamma_{\alpha} \gamma\left(\gamma \notin u \circ \Gamma_{\alpha}\right)\right\}$ is an open cover of $\Gamma$. Choose a finite subcover $A, \Gamma_{\alpha} \gamma_{1}, \ldots, \Gamma_{\alpha} \gamma_{n}$. Recall that $I_{y}=\{p \in I \mid p y=y\}, J_{y}$ $=J(I) \cap I_{y}\left(u \in J_{y}\right)$. As usual, $I_{y}=\cup_{v \in J_{y}} v I_{y}=\cup_{v \in J_{y}} v \Gamma$.

If $v \in J_{y}$ belongs to $\left(u \circ \Gamma_{\alpha}\right) \gamma_{j}$, then $u=u v \in u\left(u \circ \Gamma_{\alpha}\right) \gamma_{j}$, or $u \in$ $\left(u \circ \Gamma_{\alpha}\right) \gamma_{j}$. It follows that $\gamma_{j}^{-1} \in\left(u \circ \Gamma_{\alpha}\right) \cap \Gamma$. Now because (a) $\Gamma_{\alpha}=\Gamma_{\alpha}^{-1}$, (b) $(\Gamma, \mathfrak{F}(u))$ is an $F$-group, and (c) $\operatorname{cls}_{\mathfrak{F}(u)} \Gamma_{\alpha}=\left(u \circ \Gamma_{\alpha}\right) \cap \Gamma$, it follows that $\gamma_{j} \in\left(u \circ \Gamma_{\alpha}\right) \cap \Gamma$, contradicting our choice of $\gamma_{j}$. We conclude that $u \circ \Gamma_{\alpha} \gamma_{j}$, $j=1, \ldots, n$, contains no element of $J_{y}$.

Now define $I_{y}^{\prime}=u \circ \Gamma$. Clearly, $I_{y}^{\prime} \Gamma \subseteq I_{y}^{\prime}$, and therefore if $J_{y}^{\prime}=J_{y} \cap I_{y}^{\prime}$, $I_{y}^{\prime}=\cup_{v \in J_{\underline{y}}^{\prime}} v \Gamma$. Also, because $\pi$ is an RIC extension, $I_{y}^{\prime} x=(u \circ \Gamma) x=u$ $\circ(\Gamma x)=\pi^{-1} y$. Now certainly $I_{y}^{\prime}=u \circ \Gamma=\cup_{j=1}^{n}\left(u \circ \Gamma_{\alpha}\right) \gamma_{j} \cup u \circ A$, and so, by the result of the preceding paragraph, $J_{y}^{\prime} \subseteq u \circ A$. Since $\Gamma_{\alpha} \subseteq A$ $\subseteq u \circ \Gamma_{\alpha}, u \circ A=u \circ \Gamma_{\alpha}$, and therefore because $\left(u \circ \Gamma_{\alpha}\right) \Gamma(x) \subseteq u \circ \Gamma_{\alpha}, v \Gamma(x)$ $\subseteq u \circ \Gamma_{\alpha}$ for every $v \in J_{y}^{\prime}$.

Now suppose $\left(x, x^{\prime}\right) \in E_{\pi}^{\prime}$, and choose $v \in J_{y}^{\prime}$ with $v x^{\prime}=x^{\prime}$. Since $E_{\pi}^{\prime}$ is invariant, $u\left(x, x^{\prime}\right)=\left(x, u x^{\prime}\right) \in E_{\pi}^{\prime}$, and it follows that $u x^{\prime} \in E(x)=\Gamma(x) x$. Then $x^{\prime}=v\left(u x^{\prime}\right) \in v E(x)=v \Gamma(x) x$. Since $v \Gamma(x) \subseteq u \circ \Gamma_{\alpha}, x^{\prime} \in u \circ \alpha$, and we have proved Theorem 2.6.1.

2.6.11. Proposition. Let $\mathfrak{X} \rightarrow^{\pi}$ Oु be an arbitrary homomorphism of minimal flows, and let $W, \Gamma, x \in X(u) \cap W, N_{x}$ be as above. If $z \in \bigcap_{\alpha \in N_{x}} u \circ \alpha$, then $(x, z) \in Q_{\pi}$.

PRoof. Now it is convenient to suppose $\alpha \in N_{x}$ is of the form $\alpha=W$ $\cap \cup_{g \in V} g^{-1} U$, where also $V x \subseteq U$. To say $z \in u \circ \alpha$ is to say there are nets $h_{\nu}, g_{v}$ in $G$ and $x_{v}$ in $U$ such that (a) $g_{v}^{-1} x_{v} \in W$, (b) $h_{\nu} g_{v}^{-1} x_{v} \rightarrow z$, (c) $h_{v} \rightarrow u$, and (d) $g_{\nu} \in V$. By (a) $\left(h_{\nu} g_{\nu}^{-1} x_{\nu}, h_{\nu} x\right) \in h_{\nu}(W \times W) \subseteq R_{\pi}$. Therefore if $k_{\nu}=g_{\nu} h_{\nu}^{-1}$,

$$
k_{\nu}\left(h_{\nu} g_{\nu}^{-1} x_{\nu}, h_{\nu} x\right)=\left(x_{\nu}, g_{\nu} x\right) \in U \times U \cap R_{\pi},
$$

by (d) and our choice of $x_{v}, V$. It follows that $\left(h_{\nu} g_{\nu}^{-1} x_{\nu}, h_{\nu} x\right)$ $\in \overline{G\left(U \times U \cap R_{\pi}\right)}$. Because $U$ is an arbitrary neighborhood of $x,(z, x)$ $\in Q_{\pi}$.

Proposition 2.6.11 and Theorem 2.6.1, together with the discussion preceding the proof of the latter, combine to imply Theorem 2.6.2. It is left for us to 
prove Theorem 2.6.3. Recall that $\mathfrak{X} \rightarrow^{\pi}$ $\mathcal{Y}$ is a homomorphism of minimal flows satisfying Bronstein's condition and also $E_{\pi}=R_{\pi} \cdot \mathscr{Z} \rightarrow^{\lambda}$ Oु is a homomorphism and $(\pi, \lambda)$ satisfies the generalized Bronstein condition. $\mathscr{Z}$ is topologically transitive but possibly not minimal.

Initially we suppose $\pi$ satisfies the stronger hypothesis of being RIC. Let $A \subseteq R_{\pi, \lambda}$ be a closed invariant set with nonempty interior, and let $(x, z) \in A$ be an a.p. point, where $u(x, z)=(x, z)$. Set $y=\pi x=\lambda z$. There exist neighborhoods $U$ of $x$ and $U^{\prime}$ of $z$ such that $U \times U^{\prime} \cap R_{\pi, \lambda} \subseteq A$, and we may choose $V \subseteq G$ with $V u=V$ and $V x \subseteq U, V z \subseteq U^{\prime}$. If $x^{\prime} \in \alpha=X(u)$ $\cap \pi^{-1} y \cap \cup_{g \in V} g^{-1} U$, say $x^{\prime}=g^{-1} x^{\prime \prime}$, then

$$
\left(x^{\prime}, z\right)=g^{-1}\left(x^{\prime \prime}, g z\right) \in g^{-1}\left(U \times U^{\prime} \cap R_{\pi, \lambda}\right) \subseteq G A .
$$

It follows from Theorem 2.6.1 that

$$
u \circ(\alpha x\{z\})=(u \circ \alpha) x\{u z\}=(u \circ \alpha) x\{z\}=\pi^{-1} y x\{z\} \subseteq \overline{G A} .
$$

Now the set $Z^{\prime}=\left\{z \in Z \mid(x, z) \in A^{0}\right.$ for some $\left.x \in X\right\}$ contains $G U^{\prime}$. Since $\mathscr{Z}$ is assumed to be topologically transitive $Z^{\prime}$ is dense, and by the above $Z^{\prime \prime}=\left\{z \in Z \mid \pi^{-1} \lambda z x\{z\} \subseteq A\right\}$ is also dense. Now if $A \neq R_{\pi, \lambda}, A^{c} \cap R_{\pi, \lambda}$ is open and invariant, and the set $Z^{\prime \prime \prime}=\left\{z \in Z \mid(x, z) \in A^{c} \cap R_{\pi}\right.$ for some $\left.x\right\}$ is open and dense. Since $Z^{\prime \prime} \cap Z^{\prime \prime \prime}=\varnothing$, we have reached a contradiction, and therefore $A=R_{\pi, \lambda}$. Theorem 2.6.3 is proved for RIC extensions $\pi$.

To sketch the general case, assume $\pi$ and $(\pi, \lambda)$ satisfy the B.c. and g.B.c. We generalize $\left(2.3 .1^{\prime}\right)$ by setting $Y^{\prime}=\left\{\left(C_{\pi}(y, u), C_{\lambda}(y, u)\right) \mid y \in Y, u \in J, u y\right.$ $=y\}$. While $\mathcal{Z}$ is not necessarily minimal, it is still true that $\mathcal{O}^{\prime}$ is minimal. Furthermore, because $(\pi, \lambda)$ satisfies the g.B.c., and because homomorphisms map a.p. points to a.p. points, $\mathscr{Z}$ contains a dense set of a.p. points. This implies each $z \in Z$ belongs to at least one $C_{\lambda}(y, u)$, and therefore if we set $X^{\prime}=\left\{(x,(A, B)) \mid x \in A,(A, B) \in Y^{\prime}\right\}, Z^{\prime}=\{(z,(A, B)) \mid z \quad \in B,(A, B)$ $\left.\in Y^{\prime}\right\}$, there is a commutative diagram

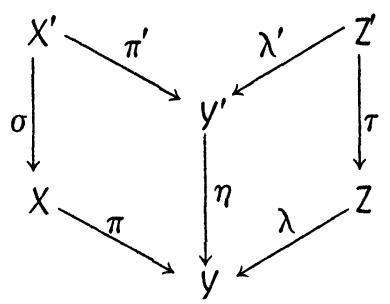

in which $\sigma, \tau$ are proximal, $\mathfrak{X}^{\prime} \rightarrow^{\pi^{\prime}} \mathcal{Y}^{\prime}$ is RIC, and $\mathscr{Z}^{\prime}$ is topologically transitive and has a dense set of a.p. points. By the remark following the statement of Theorem 2.6.3, $\left(\pi^{\prime}, \lambda^{\prime}\right)$ satisfies g.B.c. Because $\pi$ satisfies B.c. and $E_{\pi}=R_{\pi}$, we have $E_{\pi^{\prime}}=R_{\pi^{\prime}}$. By the first part of our proof above, $R_{\pi^{\prime}, \lambda^{\prime}}$ is topologically transitive. The natural generalization of Lemma 2.3 .4 implies $\sigma \times \tau\left(R_{\pi^{\prime}, \lambda^{\prime}}\right)$ $=R_{\pi, \lambda}$, and because topological transitivity is closed under homomorphisms, $R_{\pi, \lambda}$ is topologically transitive. Theorem 2.6 .3 is thereby proved.

2.7 Another proximity relation. Let $\mathfrak{X} \rightarrow^{\pi}$ Oु be a homomorphism of minimal flows. Define $U_{\pi}[x], x \in X$, to be the set of $z$ for which there exist nets $g_{\nu}$ in 
$G$ and $z_{\nu}$ in $\pi^{-1} \pi x$ such that $z_{\nu} \rightarrow z, g_{\nu} z_{\nu} \rightarrow z, g_{\nu} x \rightarrow x$. It is clear that $P_{\pi}[x] \subseteq U_{\pi}[x] \subseteq Q_{\pi}[x]$.

2.7.1. Question. Let $\mathcal{X} \rightarrow^{\pi}$ Oy satisfy the Bronstein condition. Does $U_{\pi}[x]$ $=Q_{\pi}[x]$ for all $x$ ?

It is perhaps too much to hope that the answer is "yes". On the other hand, it will develop that if for all $y \in Y, u \in J_{y}$, the set $u \pi^{-1} y$ is dense in $\pi^{-1} y$ (e.g., if $\mathcal{X}$ is $\mathscr{Y}$-distal), then the answer is "yes".

If $z \in X$, denote by $\Re_{z}$ the set of all (regular) neighborhoods of $z$ in $\pi^{-1} \pi z$. Define for each $y \in Y$ a set $\Sigma_{1}(y) \subseteq \pi^{-1} y, \Sigma_{1}(y)=\left\{z \mid u \circ U \cap u \pi^{-1} y\right.$ has nonempty $\mathfrak{F}(u)$ interior, $\left.u \in J_{y}, U \in \mathfrak{X}_{z}\right\}$. (If $u \circ U \cap u \pi^{-1} y$ has nonempty $\mathfrak{F}(u)$ interior for one $u \in J_{y}$, it does so for all $u \in J_{y}$.) $\Sigma_{1}(y)$ is closed. The argument in the lemma to follow is due to Furstenberg [50]; see also Veech [146] and Ellis [38]. Set $\Gamma=u I_{y}$ for some $u \in J_{y}$.

2.7.2. Lemma. Let $H \subseteq I_{y}$ be a closed set such that $H \Gamma=H$, and suppose every $v \in J(H)$ has the property that $v^{\prime} W \cap W \neq \varnothing, v^{\prime} \in J(H), W \in \Re_{v}$. Then $H \pi^{-1} y \subseteq \Sigma_{1}(y)$.

Proof. Fix $x \in H \pi^{-1} y$, and let $v \in J(H)$ be such that $v x=x$. If $U$ $\in \Re_{x}$, let $H_{U}=\{h \in H \mid h x \in U\}$, and note that our hypothesis implies $H_{U} \Gamma=H$. As $H$ is compact and $H_{U} \gamma$ is open, $\gamma \in \Gamma$, there exist $\gamma_{1}, \ldots, \gamma_{n}$ $\in \Gamma$ such that $\cup_{j=1}^{n} H_{U} \gamma_{j}=H$. Now if $v \in J(H), v \Gamma \cap v \circ H_{U} \gamma_{j}$ is $\mathfrak{F}(v)$ closed, and since $v \Gamma \subseteq \cup v \circ H_{U} \gamma_{j}, v \circ H_{U} \gamma_{j}$ has $\mathfrak{乛}(v)$ interior for some $j$. But $(v \Gamma, \mathfrak{F}(v))$ is an $F$-group, and therefore $v \Gamma \cap v \circ H_{U}$ has $\mathfrak{F}(v)$ interior. Now choose $\delta_{1}, \ldots, \delta_{m} \in v \Gamma$ with $\cup_{i=1}^{m} \delta_{i}\left(v \circ H_{U} \cap v \Gamma\right)=v \Gamma$, and apply similar reasoning to conclude that $v \circ U \cap v \pi^{-1} y$ has $\mathfrak{F}(v)$ interior. Thus, $x \in \Sigma_{1}(y)$ as claimed.

To construct $H$ with the properties in the lemma, regard $\left(I_{y}, \Gamma\right)$ as a (right) flow, and let $H \subseteq I_{y}$ be a $\Gamma$-minimal set. Then $H$ has the desired property (Ellis [38]). Thus, $\Sigma_{1}(y) \neq \varnothing$.

2.7.3. Lemma. Let $z \in \Sigma_{1}(y)$, and suppose $x \in \pi^{-1} y, v \in J_{y}$ are such that (a) $v x=x$, and (b) $x \in \cap_{U \in \Re_{z}} v \circ U$. Then $z \in U_{\pi}[x]$.

Proof. By (b) there exist nets $z_{\nu}$ in $\pi^{-1} y$ and $g_{\nu}$ in $G$ such that $z_{\nu} \rightarrow z, g_{\nu} z_{\nu}$ $\rightarrow x$, and $g_{v} \rightarrow v$. By (a) $g_{v} x \rightarrow v x=x$, and therefore $z \in U_{\pi}[x]$.

2.7.4. Proposition. Assume $x \rightarrow^{\pi}$ oy satisfies the Bronstein condition, and let $y \in Y$ be such that $\Sigma_{1}(y)=\pi^{-1} y$. Then for all $x \in \pi^{-1} y$ we have $U_{\pi}[x]$ $=E_{\pi}[x]=Q_{\pi}[x]$.

Proof. Fix $x \in \pi^{-1} y, v \in J_{y}$ such that $v x=x$, and let $z \in E_{\pi}[x]$. If $U \in \mathfrak{R}_{z}$, then $v \circ U \cap v \pi^{-1} y$ has $\mathfrak{F}(v)$ interior, and as it is also $\mathfrak{F}(v)$ closed, it contains $v \gamma \Gamma(z) z(\Gamma(z)$ as in \$2.6) for some $\gamma=\gamma(U) \in \Gamma$. Regard $\left\{v \gamma(U) \mid U \in \mathfrak{\Re}_{z}\right\}$ as a net, and choose a convergent subnet (regular topology) $v \gamma_{v}$, say $\lim _{\nu} v \gamma_{\nu}=p \in I_{y}$. For any $U \in \Re_{z}, v \gamma_{\nu} \Gamma(z) z \subseteq v \circ U$ for large $\nu$, and therefore $p \Gamma(z) z \subseteq v \circ U, U \in \Re_{z}$. Since $v(v \circ U) \subseteq v \circ U, v p \Gamma(z) z$ $\subseteq v \circ U, U \in \mathscr{\Re}_{z}$. If $x^{\prime}=v p \gamma z, \gamma \in \Gamma(z)$, then $z \in U_{\pi}\left[x^{\prime}\right] \subseteq Q_{\pi}\left[x^{\prime}\right]$ $=E_{\pi}\left[x^{\prime}\right]$ by Lemma 2.7.3. Since $E_{\pi}[z]=\cup_{v \in J_{y}} v \Gamma(z) z$ (\$2.6), $x=v p \gamma z$ for some $\gamma \in \Gamma(z)$ and so $z \in U_{\pi}[x]$. Since $z \in E_{\pi}[x]$ is arbitrary, the proposition is proved. 
A consequence of Lemma 2.7.2 (and the remark following it) together with Proposition 2.7.4 is

2.7.5. THEOREM. With notations as above suppose $u \pi^{-1} y$ is dense in $\pi^{-1} y$ for all $y \in Y, u \in J_{y}$. Then $E_{\pi}=U_{\pi}\left(=\left\{(x, z) \mid z \in U_{\pi}[x]\right\}\right)$. In particular, if $\mathcal{X}$ is Qy-distal, $E_{\pi}=U_{\pi}$.

In case $\mathcal{O}$ is trivial, the proposition implies

2.7.6. THEOREM. Let $\mathfrak{X}$ be a minimal flow, and suppose that $\mathfrak{X} \times \mathfrak{X}$ has a dense set of a.p. points. The equicontinuous structure relation for $\mathcal{X}$ is then $U_{\pi}$, where $\mathcal{X} \rightarrow{ }^{\pi} \Omega$ is the constant map.

Proof. In this case $\Sigma_{1}(y), \Omega=\{y\}$, is closed, invariant, nonempty, hence all of $X$. Apply the proposition.

2.7.7. Question. With notations as above let $\Sigma_{1}=\cup_{y \in Y} \Sigma_{1}(y)$. Is $\Sigma_{1}$ a Borel set? If $X$ is metrizable, is the map $y \rightarrow \Sigma_{1}(y) \in 2^{X}$ Borel? If the answer is "yes" Proposition 2.3.9 implies $\Sigma_{1}(y)=\pi^{-1} y$ for a residual set of $y$, so that $U_{\pi}[x]=E_{\pi}[x]$ for a residual set of $x$.

Now let $\beta$ be the first ordinal whose cardinal exceeds the density character of $X$. If $y \in Y, u \in J_{y}$, define closed sets $\Sigma_{\alpha}(y, u), \alpha<\beta$, inductively as follows. $\Sigma_{0}=\pi^{-1} y$. If $\Sigma_{\alpha^{\prime}}$ is defined, $\alpha^{\prime}<\alpha$, and if $\alpha$ is a limit ordinal, set $\Sigma_{\alpha}=\bigcap_{\alpha^{\prime}<\alpha} \Sigma_{\alpha^{\prime}}$. If $\alpha=\alpha^{\prime}+1, \Sigma_{\alpha}$ is the set of $z \in \Sigma_{\alpha}$ such that $u$ $\circ\left(U \cap \Sigma_{\alpha^{\prime}}\right) \cap u \pi^{-1} y$ has a point of $\Sigma_{\alpha^{\prime}}$ in its $\mathfrak{F}(u)$ interior. The remark following Lemma 2.7.2 implies $H \pi^{-1} y \subseteq \Sigma_{\alpha}$ for all $\alpha$. An argument in [146] shows $\Sigma_{\alpha}=\Sigma_{\alpha+1}$ for some $\alpha<\beta$. Denote this set by $\Sigma(y, u)$. So far as I know, this construction depends upon $u$.

2.7.8. Lemma. Let $\mathcal{X} \rightarrow^{\pi}$ Y satisfy the Bronstein condition. If $\Sigma(y, u)$ is not a perfect set, then $\mathcal{X}$ is a finite-to-one a.p. extension of of.

Proof. . If $z \in \Sigma(y, u)$ is isolated, then $\{u z\}$ has $\mathfrak{F}(u)$ interior in $u \pi^{-1} y$. This implies $\left(u \pi^{-1} y, \mathfrak{F}(u)\right)$ is $T_{2}$, and therefore $\mathcal{X}$ is an a.p. extension of $\mathscr{Y}$. But then $\pi^{-1} y=u \pi^{-1} y$, and as $u \pi^{-1}$ is compact and discrete, it is finite.

The following lemma is essentially taken from Ellis [38]. See also McMahon and $\mathrm{Wu}[90]$.

2.7.9. Lemma. Assume there exist $\alpha_{n} \in \mathcal{Q}(X)$ such that $\cap_{n=1}^{\infty} \alpha_{n} \cap R_{\pi}=\Delta$. If $E_{\pi}=R_{\pi}$, then for all $x \in X, u \in J_{y}$ such that $u x=x$, we have $x P z$ for $a$ residual set of $z \in \Sigma(\pi x, u)$.

Proof. For each $n, G\left(\alpha_{n} \cap R_{\pi}\right) \cap(\{x\} \times \Sigma(\pi x, u))$ is open dense in $\{x\}$ $\times \Sigma(\pi x, u)$. Apply the Baire category theorem.

2.7.10. THEOREM. Let $\mathfrak{X} \rightarrow{ }^{\pi}$ Y satisfy the Bronstein condition and otherwise be as in Lemma 2.7.9. Then $R_{\pi}=P_{\pi}^{3}$.

Proof. If $\left(x, x^{\prime}\right) \in R_{\pi}, u x=x$, there exists by the lemma $z \in \Sigma(\pi x, u)$ with $x P_{\pi} z$ and $u x^{\prime} P_{\pi} z$. Thus $x P_{\pi}^{2} u x^{\prime}$ and as $x^{\prime} P_{\pi} u x^{\prime}, x P_{\pi}^{3} x^{\prime}$.

2.7.11. Corollary (Ellis [38] ). Let $\mathfrak{X} \rightarrow^{\pi}$ Y be as in the theorem. If $\mathscr{X}$ has a Y-distal point, $\pi$ is an isomorphism. 
2.7.12. Corollary (Ellis, Glasner and Shapiro [ 40]). Let $\mathfrak{X} \rightarrow \pi$ y be as in the theorem. If $P_{\pi}$ is an equivalence relation (e.g., if proximal is an equivalence relation), then $\mathfrak{X}$ is a proximal extension of $\mathcal{Y}$.

2.7.13. Corollary (Ellis, Glasner and Shapiro [ 40]). Let $\mathfrak{x} \rightarrow^{\pi}$ y be as in the theorem and suppose $P_{\pi}[x]$ is countable (e.g., the proximal cell of $x$ is countable) for some $x$. Then $\pi$ is an isomorphism.

Proof. If $u x=x$, and if $\Sigma(\pi x, u)$ is a perfect set, then any residual subset of $\Sigma(\pi x, u)$ is uncountable. As $P_{\pi}[x] \cap \Sigma(\pi x, u)$ is residual, $\Sigma(\pi x, u)$ is not perfect, and by Lemma 2.7.8, $\mathcal{X}$ is an a.p. extension of $\mathcal{Y}$. Since $R_{\pi}=E_{\pi}=\Delta$, $\pi$ must be an isomorphism.

2.8 The canonical tower. Ellis, Glasner and Shapiro [40] attach to an arbitrary minimal flow a canonical tower of minimal flows, the relativized version of which I shall sketch here. In the case that $\mathcal{X}$ is a point distal flow with metrizable phase space and a residual set of distal points, the EllisGlasner-Shapiro tower reduces to the tower constructed in [146]; when further $x$ is distal, both towers reduce to Furstenberg's [50].

Let $\mathcal{X} \rightarrow^{\pi} \mathcal{Y}$ be a fixed homomorphism of minimal flows, and let $y \in Y, u$ $\in J_{y}, x \in \pi^{-1} y$ be fixed with $u x=x$. The construction below, which is taken from [40], [54], can be shown not to depend on the choices of $y, u, x$, and $I \subseteq S$. Let $W=u \pi^{-1} y, \Gamma=u I_{y}$, and let $\beta$ again be greater than the density character of $X$. Define sets $A_{\alpha}, x \in A_{\alpha} \subseteq W, \alpha<\beta$ by $A_{0}=W$, and if $A_{\alpha^{\prime}}$ is defined for $\alpha^{\prime}<\alpha$,

$$
\begin{aligned}
& A_{\alpha}=\bigcap_{\alpha^{\prime}<\alpha} A_{\alpha^{\prime}} \quad(\alpha=\text { limit ordinal }) \\
& A_{\alpha}=\bigcap_{U \in N_{x}} \operatorname{cls}_{\mathfrak{q}(u)}\left(U \cap A_{\alpha^{\prime}}\right) \quad\left(\alpha=\alpha^{\prime}+1\right)
\end{aligned}
$$

where $N_{x}$ are the $\mathfrak{F}(u)$ neighborhoods of $x$. Since $x \in A_{\alpha}, A_{\alpha} \neq \varnothing$ for each $\alpha$. As in the last section there is an ordinal $\alpha<\beta$ with $A_{\alpha}=A_{\alpha+1}$. The least such $\alpha$ is denoted $\alpha(\pi)$.

Let $Z=\left(2^{X}\right)^{\alpha(\pi)}$, and define $\omega_{\alpha} \in Z, \alpha \leqslant \alpha(\pi)$ by

$$
\omega_{\alpha}\left(\alpha^{\prime}\right)= \begin{cases}u \circ A_{\alpha^{\prime}}, & \alpha^{\prime} \leqslant \alpha, \\ u \circ A_{\alpha}, & \alpha^{\prime}>\alpha .\end{cases}
$$

Since $u \omega_{\alpha}=\omega_{\alpha}, \mathscr{Y}_{\alpha}=\left(G, \mathcal{O}\left(\omega_{\alpha}\right)\right)$ is a minimal flow. The assignment $\sigma_{\alpha \alpha^{\prime}}\left(g \omega_{\alpha}\right)$

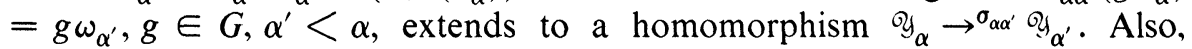
define $x_{\alpha} \in X \times Z$ by $x_{\alpha}=\left(x, \omega_{\alpha}\right)$, and let $x_{\alpha}=\left(G, O\left(x_{\alpha}\right)\right)$. $x_{\alpha}^{\alpha}$ is also minimal, and there are natural (coordinate) homomorphisms $\mathfrak{o x}_{\alpha} \rightarrow{ }^{\pi_{\alpha}} \mathscr{g}_{\alpha}, \mathfrak{x}_{\alpha}$ $\rightarrow^{\tau_{\alpha}}$ X. For each $\alpha, \tau_{\alpha}$ is proximal and $\pi_{\alpha}$ is RIC (see [40]). Setting $\dddot{z}_{\alpha}$ $=\mathfrak{X}_{\alpha} / E_{\pi_{\alpha}}\left(=\mathfrak{x}_{\alpha} / Q_{\pi_{\alpha}}\right.$ by Theorem 2.6.2) there is a natural diagram

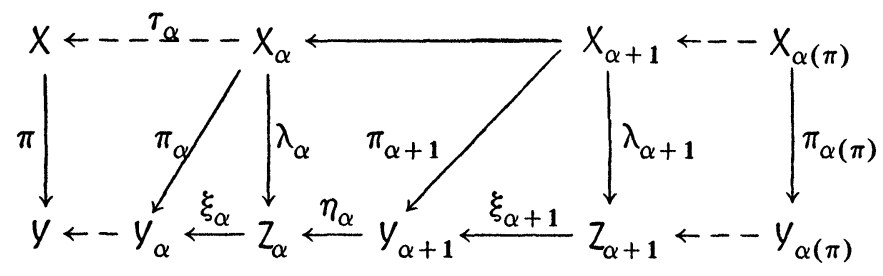


in which $\mathscr{Z}_{\alpha}$ is an a.p. extension of $\mathscr{Y}_{\alpha}$ and $\mathscr{Y}_{\alpha+1}$ a proximal extension of $\mathscr{Z}_{\alpha}$ for each $\alpha$. Thus, $\mathscr{Y}_{\alpha(\pi)}$ is a PI extension of $\mathscr{Y}_{\text {. As }} E_{\pi_{\alpha(\pi)}}=R_{\pi_{\alpha(\pi)}}$, Theorem 2.6.3 implies $\mathfrak{X}_{\alpha(\pi)}$ is a weak mixing extension of $\mathscr{Y}_{\alpha(\pi)}$. Also, $\mathfrak{X}_{\alpha(\pi)}$ is a proximal extension of $\mathcal{X}$. Thus, Theorem 2.1.3 is proved. $(\alpha(\pi)$ is countable if $X$ is metrizable, in which case $X_{\alpha(\pi)}$ is also metrizable.) Now it can also be shown that the diagram

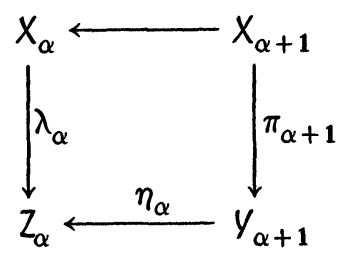

is the same as $\left(2.3 .1^{\prime}\right)$. Therefore, if $X$ is metrizable or $G \sigma$-compact, and if $\mathscr{X}$ has a $\mathcal{Y}$-distal point, Corollary 2.3.6 and the remark preceding Lemma 2.3.8 imply that for each $\alpha, \mathfrak{X}_{\alpha+1}$ is an h.p. extension of $\mathfrak{X}_{\alpha}$ and $\mathscr{Y}_{\alpha+1}$ an h.p. extension of $\mathscr{Z}_{\alpha}$. The projective limit of h.p. extensions is also an h.p. extension, and Theorem 2.1.5 follows. (That $\pi_{\alpha(\pi)}$ is an isomorphism is Ellis' Corollary 2.7.11.)

2.8.1 Question. Let $\mathfrak{X}$ be a point distal flow. Does $\mathcal{X}$ have an invariant Borel probability measure?

The question is open even for a.a. flows (which are point-distal). If $\mathfrak{X}$ is distal, the answer is "yes" and was obtained by Furstenberg using his structure theorem [50]. This result may be used to generalize the fixed point theorem of Ryll-Nardzewski [118] as follows (see [137]):

2.8.2. THEOREM. Let $X$ be a weakly compact subset of a Banach space $B$, and let $\Sigma$ be a semigroup of bounded linear operators on $B$ which leaves $X$ invariant. If $\Sigma$ is norm distal on $X$, the closed convex hull of $X$ contains a fixed point for $\Sigma$.

Appendix. In this Appendix I shall prove Theorem 2.2.1, the discrete case of which is due to Ellis.

Suppose $g \in G, p \in S(G)$ are such that $g p=p$. We are to prove $g=e$, to which end we let $g_{v}$ be a net in $G$ convergent to $p$. The assumption $g p=p$ implies $\lim _{\nu}\left(f\left(g g_{\nu}\right)-f\left(g_{\nu}\right)\right)=0, f \in \mathcal{C}$.

Let $U$ be a compact symmetric neighborhood of $e$, and use Zorn's lemma to find a set $A \subseteq G$ which is maximal for the property $U a \cap A=\{a\}, a \in A$. Notice that if $x \in G$, then $x \in U a$ for some (possibly not unique) $a \in A$. Thus, we may write $g_{\nu}=\alpha_{\nu} a_{\nu}, \alpha_{\nu} \in U, a_{\nu} \in A$. Passing to a subnet if necessary, assume $\lim _{v} \alpha_{v}=\alpha \in U$ exists.

Let $B=\alpha A=\{\alpha a \mid a \in A\}$, where $\alpha$ is as above. If $V$ is a neighborhood of $e$ such that $V=V^{-1}$ and $\alpha^{-1} V^{2} \alpha \subseteq U$, then for all $b \in B, V b \cap V B=\{b\}$. Fix such a $V$, and let $b_{v}=\alpha a_{v}$, where $a_{\nu}$ is as in the preceding paragraph. Since $\lim _{\nu} b_{\nu}\left(\alpha_{\nu} a_{\nu}\right)^{-1}=e$ and $g_{\nu}=\alpha_{\nu} a_{\nu}$, we have $\lim _{\nu} g_{\nu} b_{\nu}^{-1}=e$. In particular, if $f \in \mathcal{C}$,

$$
\lim _{\nu} f\left(b_{\nu}\right)=\lim _{\nu} f\left(g_{\nu}\right)=\lim _{\nu} f\left(g g_{\nu}\right)=\lim _{\nu} f\left(g b_{\nu}\right) .
$$

There are two cases to consider. 
Case 1. There exist a neighborhood $W$ of $e$ and an index $\nu_{0}$ such that $g g_{v} \notin W B$ if $\nu \geqslant \nu_{0}$. We may suppose $W \subseteq V$ so that if $b, b^{\prime} \in B$ are distinct points, $W b \cap W b^{\prime}=\varnothing$. Let $\varphi \geqslant 0$ be a continuous function on $G$ such that $\varphi(e)=1$ and $\varphi \equiv 0$ on $W^{c}$. Define $f \in \mathcal{C}$ by

$$
f(x)= \begin{cases}\varphi\left(x b^{-1}\right), & x \in W b, b \in B \\ 0 & \text { otherwise. }\end{cases}
$$

A moment's reflection shows $f(x)=\max _{b \in B} \varphi\left(x b^{-1}\right)$ for all $x$, and as the family $\left\{\varphi\left(x b^{-1}\right) \mid b \in B\right\}$ is jointly 1.u.c., $f$ is also l.u.c. Since $f\left(b_{v}\right) \equiv 1$ and $f\left(g b_{v}\right) \equiv 0$, we have reached a contradiction.

Case 2. Passing to a subnet if necessary, assume $b_{v}^{\prime} \in B$ are such that $\lim _{\nu} g g_{\nu}\left(b_{\nu}^{\prime}\right)^{-1}=e$, so that also $\lim _{\nu} g b_{\nu}\left(b_{\nu}^{\prime}\right)^{-1}=e$. Notice that $\lim _{\nu}\left(f\left(b_{\nu}\right)\right.$ $\left.-f\left(b_{v}^{\prime}\right)\right)=\lim _{\nu}\left(f\left(g b_{\nu}\right)-f\left(b_{v}^{\prime}\right)\right)=0$. This will be seen to be impossible unless $g=e$. Call a finite or infinite sequence from $B$ a string if either (a) $\Sigma=\{b\}$ is a singleton, or (b) if whenever $b^{\prime}$ follows $b$ in $\Sigma$, then $g b \in V b^{\prime}$. From our choice of $V$ it follows that for each $b \in B$ there is at most one $b^{\prime}$ such that $\left(b, b^{\prime}\right)$ is a string. Decreasing $V$ if necessary so that $g^{-1} V^{2} g \subseteq U$, there exists at most one $b^{\prime \prime} \in B$ such that $\left(b^{\prime \prime}, b\right)$ is a strong. These remarks imply the set of maximal strings partitions $B$ (although strictly speaking a string $\Sigma$ is not a subset of $B$ because $\Sigma$ may be periodic).

If $g \neq e$, we may choose $V$ small enough that $(b, b)$ is not a string, and therefore in a maximal string $\Sigma$, successive elements are distinct. Because of this, there exists on $\Sigma$ a $\{0,1,2\}$-valued function $\Psi$ with the properties that (i) $\Psi=1$ if $\Sigma$ is a singleton, (ii) $\left|\Psi\left(b^{\prime}\right)-\Psi(b)\right| \geqslant 1$ if $b, b^{\prime}$ are successive elements of $\Sigma$, and (iii) if $\Sigma$ is periodic, $\Psi$ has the same period. Now "coalesce" the different $\Psi$ 's into a function on $B$. If $b, b^{\prime} \in B$ are such that $g b \in V b^{\prime}$, then $\left(b, b^{\prime}\right)$ are successive elements in some maximal string, and $\left|\Psi(b)-\Psi\left(b^{\prime}\right)\right|$ $\geqslant 1$. Now let $\varphi$ be as in Case 1 (taking $W=V$ ). Define $f$ on $G$ this time as $f(x)=\max _{b \in B} \Psi(b) \varphi\left(x b^{-1}\right)$. Again $f \in \mathcal{C}$. By our choice of $b_{\nu}, b_{\nu}^{\prime}$ we have $g b_{\nu} \in V b_{\nu}^{\prime}$ for large $\nu$, and therefore $\left(b_{\nu}, b_{\nu}^{\prime}\right)$ are successive elements in a maximal string for large $\nu$. By the choice of $b_{\nu}, b_{\nu}^{\prime}, \Psi$, and $f$ we have

$$
\begin{aligned}
0 & =\lim _{\nu}\left|f\left(g g_{\nu}\right)-f\left(g_{\nu}\right)\right|=\lim _{\nu}\left|f\left(g b_{\nu}\right)-f\left(b_{\nu}\right)\right| \\
& =\lim _{\nu}\left|f\left(b_{\nu}^{\prime}\right)-f\left(b_{\nu}\right)\right| \geqslant 1 .
\end{aligned}
$$

This is a contradiction and therefore $g=e$.

\section{BIBLIOGRAPHY}

1. R. L. Adler, F-expansion revisited, Recent Advances in Topological Dynamics (Proc. Conf., New Haven, Conn., 1972), Lecture Notes in Math., vol. 318, Springer-Verlag, Berlin and New York, 1973, pp. 1-5. MR 51 \#6880.

2. H. Anzai, Ergodic skew product transformations on the torus, Osaka Math. J. 3 (1951), 83-99. MR 12, 719.

3. V. I. Arnol'd, Small denominators and problems of stability of motion in classical and celestial mechanics, Uspehi Mat. Nauk. 18 (1963), no. 6(114), 91-192 = Russian Math. Surveys 18 (1963), no. 6, 85-191. MR 30 \#943.

4. J. Auslander, Endomorphisms of minimal sets, Duke Math. J. 30 (1963), 605-614. MR 27 \#5245. 
5. J. Auslander, On the proximal relation in topological dynamics, Proc. Amer. Math. Soc. 11 (1960), 890-895. MR 29 \#1632.

6. - Regular minimal sets. I, Trans. Amer. Math. Soc. 123 (1966), 469-479. MR 33 \# 1845.

7. J. Auslander and S. Glasner, Distal and highly proximal extensions of minimal flows, Univ. of Maryland, 39 pp. (preprint).

8. J. Auslander and F. Hahn, Point transitive flows, algebras of functions, and the Bebutov system, Fund. Math. 60 (1967), 117-137. MR 36 \#4541.

9. L. Auslander, L. Green and F. Hahan, Flows on homogeneous spaces, Ann. of Math. Studies, no. 53, Princeton Univ. Press, Princeton, N.J., 1963. MR 29 \#4841.

10. L. Benzinger, Uniformly distributed sequences in locally compact groups. I, II, Trans. Amer. Math. Soc. 188 (1974), 149-165; ibid, 188 (1974), 167-178. MR 48 \# 11388.

11. I. D. Berg, M. Rajagopolan and L. Rubel, Uniform distribution in locally compact Abelian groups, Trans. Amer. Math. Soc. 133 (1968), 435-446. MR 37 \# 3279.

12. G. Birkhoff, Dynamical systems, Amer. Math. Soc. Colloq. Publ., vol. 9, Amer. Math. Soc., Providence, R.I., 1927.

13. J. R. Blum and D. L. Hanson, On invariant probability measures. I, Pacific J. Math. 10 (1960), 1125-1129. MR 24 \# A3260.

14a. S. Bochner, $A$ new approach to almost periodicity, Proc. Nat. Acad. Sci. U.S.A. 48 (1962), 2039-2043. MR 26 \#2816.

14b. H. Bohr, Zur Theorie der fastperiodischen Funktionen. I, II, III, Acta. Math. 45 (1925), 29-127; ibid 46 (1925), 101-214; ibid 47 (1926), 237-281.

15. E. Borel, Les probabilités dénombrables et leurs applications arithmétiques, Rend. Circ. Mat. Palermo 27 (1909), 247-271.

16. N. Bourbaki, Éléments de mathématique, Fasc. 29, Livre VI: Intégration, Chap. 7, Actualitiés Sci. Indust., no. 1306, Hermann, Paris, 1963. MR 31 \#3539.

17. —_ Eléments de mathématique, Fasc. 8, Livre III: Topologie générale., 2nd ed., Actualitiés Sci.Indust., no. 1045, Hermann, Paris, 1958. MR 30 \#3439.

18. R. Bowen, Equilibrium states and the ergodic theory of Anosov diffeomorphisms, Lecture Notes in Math., vol. 470, Springer-Verlag, Berlin and New York, 1975.

19a. Periodic points and measures for Axiom A diffeomorphisms, Trans. Amer. Math. Soc. 154 (1971), 377-397. MR 43 \# 8084.

19b. , Periodic orbits for hyperbolic flows, Amer. J. Math. 94 (1972), 1-30. MR 45 \#7749.

20. I. Bronštein, $A$ theorem on the structure of almost distal expansions of minimal sets, Math. Issled. 6 (1971), 22-32. (Russian) MR 44 \#7532.

21. D. C. Champernowne, The construction of decimals normal in the scale of 10 , J. London Math. Soc. 8 (1933), 254-260.

22. J. Clay, Proximity relations in transformation groups, Trans. Amer. Math. Soc. 108 (1963), 88-96. MR 27 \#4218.

23. C. M. Colebrook, The Hausdorff dimension of certain sets of nonnormal numbers, Michigan Math. J. 17 (1970), 103-116. MR 41 \#5321.

24. J. P. Conze et M. Keane, Ergodicité d'un flot cylindrique, C. R. Acad. Sci. Paris (to appear).

25. A. H. Copeland and P. Erdös, Note on normal numbers, Bull. Amer. Math. Soc. 52 (1946), 857-860. MR 8, 194.

26. H. Davenport and P. Erdös, Note on normal decimals, Canad. J. Math. 4 (1952), $58-63$. MR 13, 825.

27. K. deLeeuw and I. Glicksberg, Applications of almost periodic compactifications, Acta Math. 105 (1961), 63-97. MR 24 \#A1632.

28. M. Denker and E. Eberlein, Ergodic flows are strictly ergodic, Advances in Math. 13 (1974), 437-473. MR 50 \# 4890.

29. W. F. Eberlein, Abstract ergodic theorems and weakly almost periodic functions, Trans. Amer. Math. Soc. 67 (1949), 217-240. MR 12, 112.

30. B. Eckmann, Uber monothetische Gruppen, Comment. Math. Helv. 16 (1944), 249-263. MR 6, 146.

31. H. G. Eggleston, The fractional dimension of a set defined by decimal properties, Quart. J. Math. Oxford Ser. 20 (1949), 31-36. MR 11, 88. 
32. R. Ellis, A semigroup associated with a transformation group, Trans. Amer. Math. Soc. 94 (1960), 272-281. MR 23 \# A961.

33. - Distal transformation groups, Pacific J. Math. 8 (1958), 401-405. MR 21 \# 96.

34. - Lectures on topological dynamics, Benjamin, New York, 1969. MR 42 \#2463.

35. - Locally compact transformation groups, Duke Math. J. 24 (1957), 119-125. MR 19, 561.

36. - Point transitive transformation groups, Trans. Amer. Math. Soc. 101 (1961), 384-395. MR 24 \#A1119.

37. - The structure of group-like extensions of minimal sets, Trans. Amer. Math. Soc. 134 (1968), 261-287. MR 38 \#6569.

38. - The Veech structure theorem, Trans. Amer. Math. Soc. 186 (1973), 203-218. MR 50 \#3204.

39. - Universal minimal sets, Proc. Amer. Math. Soc. 11 (1960), 540-543. MR 22 \# 8491.

40. R. Ellis, S. Glasner and L. Shapiro, 99 flows (preprint, Univ. of Minnesota), Advances in Math. (to appear).

41. R. Ellis and W. H. Gottschalk, Homomorphisms of transformation groups, Trans. Amer. Math. Soc. 94 (1960), 258-271. MR 23 \# A960.

42. R. Ellis and $\mathrm{H}$. B. Keynes, $A$ characterization of the equicontinuous structure relation, Trans. Amer. Math. Soc. 161 (1971), 171-183. 43 \#8069.

43. - Bohr compactifications and a result of Folner, Israel J. Math. 12 (1972), 314-330. MR 47 \# 1992.

44. P. Erdös, Problems and results on diophantine approximations, Compositio Math. 16 (1964),

52-65. MR 31 \#3382.

45. E. Esclangon, Les fonctions quasi-periodiques, Thèse, Paris, 1904.

46. H. Furstenberg, A Poisson formula for semi-simple Lie groups, Ann. of Math. (2) 77 (1963), 335-383. MR 26 \#3820; errata. 28, p. 1246.

47. - Boundary theory and stochastic processes on homogeneous spaces, Proc. Sympos. Pure Math., vol. 26, Amer. Math. Soc., Providence, R.I., 1973, pp. 193-229.

48. - Disjointness in ergodic theory, minimal sets, and a problem in diophantine approximation, Math. Systems Theory 1 (1967), 1-49. MR 35 \#4369.

49. _ Strict ergodicity and transformations of the torus, Amer. J. Math. 88 (1961), 573-601. MR 24 \# A3263.

50. - The structure of distal flows, Amer. J. Math. 85 (1963), 477-515. MR 28 \#602.

51. H. Furstenberg, H. Keynes and L. Shapiro, Prime flows in topological dynamics, Israel J. Math. 14 (1973), 26-38. MR 47 \#9588.

52. S. Glasner, A metric minimal flow whose enveloping semigroup contains finitely many minimal ideals is 99, Tel-Aviv Univ., 11 pp. (preprint).

53. — Compressibility properties in topological dynamics, Amer. J. Math. (to appear).

54. —, Proximal flows, Lecture Notes in Math., vol. 517, Springer-Verlag, Berlin and New York, 1976.

55. - Relatively invariant measures, $7 \mathrm{pp}$. (preprint).

56. - Topological dynamics and group theory, Trans. Amer. Math. Soc. 187 (1974), 327-334. MR 49 \# 1496.

57. R. Godement, Les fonctions de type positif et la théorie des groupes, Trans. Amer. Math. Soc. 63 (1948), 1-84. MR 9, 327.

58. W. H. Gottschalk and G. A. Hedlund, Topological dynamics, Amer. Math. Soc. Colloq. Publ., vol. 36, Amer. Math. Soc., Providence, R.I., 1955. MR 17, 650.

59. P. R. Halmos, Lectures on ergodic theory, Chelsea, New York, 1960. MR 22 \#2677.

60. S. Hartman, Remarks on equidistribution on non-compact groups, Compositio Math. 16 (1964), 66-71. MR 30 \# 184.

61. F. Hausdorff, Mengenlehre, 3rd ed., de Gruyter, Berlin, 1935.

62. E. Hecke, Analytische Funktionen und die Verteilung von Zahlen mod. Eins, Abh. Math. Sem. Univ. Hamburg 1 (1922), 54-76.

63. G. A. Hedlund, Fuchsian groups and transitive horocycles, Duke Math. J. 2 (1936), 530-542.

64. E. Hlawka, Ein matrisches Gegenstück zu einem Satz von W. A. Veech, Monatsh. Math. 76 (1972), 436-447. MR 48 \#263. 
64a. E. Hlawaka, Zur formalen Theorie der Gleichverteilung in kompakten Gruppen, Rend. Circ. Mat. Palermo (2) 4 (1955), 33-47. MR 17, 594.

65. K. Jacobs, Lipschitz functions and the prevalence of strict ergodicity for continuous-time flows, Contributions to Ergodic Theory and Probability (Proc. Conf., Ohio, 1970), Lecture Notes in Math., vol. 160, Springer-Verlag, Berlin and New York, 1970, pp. 87-124. MR 43 \#471.

66. R. I. Jewett, The prevalence of uniquely ergodic systems, J. Math. Mech. 19 (1969/70), 717-729. MR 40 \#5824.

67. S. Kakutani, Induced measure preserving transformations, Proc. Imp. Acad. Tokyo 19 (1943), 635-641. MR 7, 255.

68. T. Kamae, Subsequences of normal sequences, Israel J. Math. 16 (1973), 121-149. MR 49 \#3086.

69. T. Kamae and B. Weiss, Normal numbers and selection rules, Israel J. Math. 21 (1975), 101-110.

70. A. B. Katok and A. M. Stepin, Approximations in ergodic theory, Uspehi Mat. Nauk 22 (1967), no. 5 (137), 81-106 = Russian Math. Surveys 22 (1967), no. 5, 77-102. MR 36 \#2776.

71. M. Keane, Coding problems in ergodic theory, Univ. of Rennes, $24 \mathrm{pp}$. (preprint).

72. - Interval exchange transformations, Math. Z. 141 (1975), 25-31. MR 50 \# 10207.

73. - Non-ergodic interval exchange transformations, Univ. of Rennes, $15 \mathrm{pp}$. (preprint).

74. - Strongly mixing g-measures, Invent. Math. 16 (1972), 309-324. MR 46 \#9295.

75. - Polygonal geodesic flows, Preprint.

76. H. Kesten, On a conjecture of Erdös and Szüsz related to uniform distribution mod 1, Acta. Arith. 12 (1966/67), 193-212. MR 35 \# 155.

77. H. Keynes and D. Newton, A minimal, non-uniquely ergodic interval exchange transformation, Univ. of Minnesota (preprint).

78. - Real prime flows, Univ. of Minnesota, $28 \mathrm{pp}$. (preprint).

79. H. Keynes and J. B. Robertson, Eigenvalue theorems in topological transformation groups, Trans. Amer. Math. Soc. 139 (1969), 359-369. MR 38 \#6029.

80. A. Ya. Khinchin, Continued fractions, Univ. of Chicago Press, Chicago, 1964. MR 28 \#5037.

81. A. W. Knapp, Decomposition theorem for bounded uniformly continuous functions on a group, Amer. J. Math. 88 (1966), 902-914. MR 34 \#8212.

82. — Distal functions on groups, Trans. Amer. Math. Soc. 128 (1967), 1-40. MR 36 \#5923.

83. - Functions behaving like almost automorphic functions Topological dynamics (Sympos., Colorado, 1967), edited by J. Auslander and W. Gottschalk, Benjamin, New York, 1968, pp. 299-317. MR 38 \#6570.

84. W. Krieger, On generators in ergodic theory, Internat. Congress Math. (Vancouver, B.C., 1974), vol. 2, Canad. Math. Congress, 1975, pp. 303-308.

85. —, On unique ergodicity, Proc. Sixth Berkeley Sympos. Math. Statist. and Prob.

(Berkeley and Los Angeles), vol. 11, Univ. California Press, Berkeley, 1972, pp. 327-346.

86. N. Krylov and N. Bogoliouboff, La théorie générale de la mesure dans son application à l'étude des système dynamiques non linéaires, Ann. of Math. (2) 38 (1937), 65-113.

87. D. McMahon, On the role of an abelian phase group in relativized problems in topological dynamics, 34 pp. (preprint).

88. - Weak mixing and a note on a structure theorem for minimal transformation groups, Illinois J. Math. 20 (1976), 186-197.

89. D. McMahon and T. S. Wu, Equicontinuous structure relations of minimal transformation groups, Case-Western Reserve, 31 pp. (preprint).

90. - On proximal and distal extensions of minimal sets, Bull. Inst. Math. Acad. Sinica 2 (1974), no. 1, 93-107. MR 51 \# 1791.

91. - Relative equicontinuity and its variations, Case Western Reserve Univ., 23 pp. (preprint).

92. - On weak mixing and local almost periodicity, Duke Math. J. 39 (1972), 333-343. MR 49 \# 1497.

93. M. Mendes-France, Les ensembles de Bésineau, Séminaire Delange-Poitou-Pisot, Année 1973-74, No. 7, Secrétariat mathématique, Paris. 
94. R. von Mises, Wahrscheinlichkeit, Statistik und Wahrheit, Springer-Verlag, Berlin, 1928; 2nd ed., 1936.

95. I. Namioka, Right topological groups, distal flows, and a fixed point theorem, Univ . of Washington, $36 \mathrm{pp}$. (preprint).

96. - The separate continuity and the joint continuity, Univ. of Washington, $33 \mathrm{pp}$. (preprint).

97. J. von Neumann, Almost periodic functions in a group, Trans. Amer. Math. Soc. 36 (1934), 445-492.

98. I. Niven, Uniform distribution of sequences of integers, Trans. Amer. Math. Soc. 98 (1961), 52-61. MR 22 \# 10971.

99. A. Ostrowski, Notiz zur Theorie der Diophantischen Approximationen und zur Theorie der linearen Diophantischen Approximationen, Jber. Deutsch Math. Verein 36 (1927), 178-180; ibid 39 (1930), 34-46.

100. J. C. Oxtoby, Ergodic sets, Bull. Amer. Math. Soc. 58 (1952), 116-136. MR 13, 850.

101. - On two theorems of Parthasarathy and Kakutani concerning the shift transformation, Ergodic Theory (Proc. Internat. Sympos., Tulane Univ., 1961), edited by Fred B. Wright, Academic Press, New York, 1963, pp. 203-215. MR 28 \#4084.

102. W. Parry, Intrinsic Markov chains, Trans. Amer. Math. Soc. 112 (1964), 55-66. MR 28 \#4579.

103. K. R. Parthasarathy, On the category of ergodic measures, Illinois J. Math. 5 (1961), 648-656. MR 26 \#6354.

104. R. Peleg, Weak disjointness of transformation groups, Proc. Amer. Math. Soc. 33 (1972), 165-170. MR 45 \#7694.

105. K. E. Petersen, Disjointness and weak mixing of minimal sets, Proc. Amer. Math. Soc. 24 (1970), 278-280. MR 40 \#3522.

106. - Introductory ergodic theory, Univ. of North Carolina, $141 \mathrm{pp}$. (preprint).

107. On a series of cosecants related to a problem in ergodic theory, Compositio Math. 26 (1973), 313-317. MR 48 \#4273.

108. K. Petersen and L. Shapiro, Induced flows, Trans. Amer. Math. Soc. 177 (1973), 375-390. MR 48 \# 1200.

109. R. R. Phelps, Lectures on Choquet's theorem, Van Nostrand, Princeton, N.J., 1966. MR 33 \#1690.

110. G. Rauzy, Equirepartitión et entropie, Lecture Notes in Math., vol. 475, Springer-Verlag, Berlin and New York, 1975.

111. - Nombres normaux et processus déterministes, Journées Arith. de Bordeaux (Conf., Univ. Bordeaux, 1974), Astérisque, nos. 24-25, Soc. Math. France, Paris, 1975, pp. 263-265. MR 51 \#8065.

112. - Propriétés statistiques de suites arithmétiques, Presses Universitaires de France, 1976.

113. Sur une suite liée a la discrepance de la suite $(n \alpha)_{n \in N}$, Univ. d'Aix-Marseille II, 5 pp. (preprint).

114. H. Rindler, Gleichverteilte Folgen in local Kompakten Gruppen (preprint).

115. - Uniform distribution on locally compact groups (to appear).

116. V. A. Rohlin, Exact endomorphisms of a Lebesgue space, Izv. Akad. Nauk SSSR Ser. Mat. 25 (1961), 499-530; English transl., Amer. Math. Soc. Transl. (2) 39 (1964), 1-36. MR 26 \# 1423.

117. L. A. Rubel, Uniform distribution in locally compact groups, Comment. Math. Helv. 39 (1965), 253-258. MR 31 \#3537.

118. C. Ryll-Nardzewski, On fixed points of semigroups of endomorphisms of linear spaces, Proc. Fifth Berkeley Sympos. Math. Statist. and Prob. (Berkeley, Calif., 1965/66), Vol. II, part 1, Univ. California Press, Berkeley, Calif., pp. 55-61. MR 35 \# 5977.

119. R. Sacksteder, On the convergence to invariant measures, $\mathrm{Z}$. Wahrscheinlichkeitstheorie und Verw. Gebiete (to appear).

120. - The measures invariant under an expanding map, Lecture Notes in Math., vol. 392, Springer-Verlag, Berlin and New York, 1974.

121. K. Schmidt, A cylinder flow arising from irregularity of distribution, Univ. of Warwick, 1975,12 pp. (preprint).

122. - Every ergodic transformation has nonatomic invariant measures, Univ. of Warwick, $1975,12 \mathrm{pp}$. (preprint). 
123. K. Schmidt, Infinite invariant measures on the circle, Univ. of Warwick, 1975, 9 pp. (preprint).

124. W. M. Schmidt, Irregularities of distribution, Quart. J. Math. Oxford Ser. (2) 19 (1968), 181-191. MR 37 \#4029.

125. - Irregularities of distribution. VI, Compositio Math. 24 (1972), 63-74. MR 47 \# 152.

126. - Irregularities of distribution. VIII, Trans. Amer. Math. Soc. 198 (1974), 1-22. MR 50 \# 12952.

127. L. Shapiro, Distal and proximal extensions of minimal flows, Math. Systems Theory 5 (1971), 76-88. MR 46 \#877.

128. - On the structure of minimal flows, Global Differentiable Dynamics, (Proc. Conf., Case Western Reserve Univ., 1969), Lecture Notes in Math., vol. 235, Springer-Verlag, Berlin and New York, 1971, pp. 123-128. MR 50 \# 5089.

129. __ Proximality in minimal transformation groups, Proc. Amer. Math. Soc. 26 (1970), 521-525. MR 42 \# 1091.

130. - Regularities of distribution, Univ. of Minnesota, 1975, 33 pp. (preprint).

131. - Regularities of distribution on the torus, Univ. of Minnesota, 9 pp. (preprint).

132. K. Sigmund, Generic properties of invariant measures for Axiom A diffeomorphisms, Invent. Math. 11 (1970), 99-109. MR 44 \#3349.

133. - On dynamical systems with the specification property, Trans. Amer. Math. Soc. 190 (1974), 285-299. MR 50 \# 4898.

134. Ja. G. Sinai, Introduction to ergodic theory, Erivan State Univ., Erivan, 1973. (Russian)

135. H. Totoki, Ergodic theory, Lecture Note Ser., no. 14, Mat. Inst., Aarhus Univ., Aarhus, 1969. MR 40 \#425.

136. W. A. Veech, Q-almost automorphic functions, Problems in Analysis, edited by R. C. Gunning, Princeton Univ. Press, Princeton, N.J., 1970, pp. 345-351. MR 50 \# 5368.

137. - A fixed point theorem-free approach to weak almost periodicity, Trans. Amer. Math. Soc. 177 (1973), 353-362. MR 49 \# 7998.

138. - Almost automorphic functions on groups, Amer. J. Math. 87 (1965), 719-751. MR 32 \#469.

139. - Almost automorphy and a theorem of Loomis, Arch. Math. (Basel) 18 (1967), 267-270. MR 36 \# 1925.

140. - Applications of ergodic theory to some problems of uniform distribution, Proc. Conf. on Ergodic Theory and Topological Dynamics (Univ. of Kentucky, 1971), Math. Dept., Univ. of Kentucky, Lexington, Ky., pp. 26-33.

141. Complementation and continuity in spaces of almost automorphic functions, Math. Scand. 25 (1969), 109-112. MR 41 \#737.

142. - Finite group extension of irrational rotations, Israel J. Math. 21 (1975), 240-259.

143. - Generalizations of almost periodic functions, Unpublished notes, $58 \mathrm{pp}$.

144. - Minimal sets and Souslin sets, Recent Advances in Topological Dynamics (Proc.

Conf., Yale Univ., 1972), edited by A. Beck, Lecture Notes in Math., vol. 318, Springer-Verlag, Berlin and New York, 1973, pp. 253-266.

145. - On a theorem of Bochner, Ann. of Math. (2) 86 (1967), 117-137. MR 35 \#6780.

146. - Point-distal flows, Amer. J. Math. 92 (1970), 205-242. MR 42 \#2462.

147. - Properties of minimal functions on abelian groups, Amer. J. Math. 91 (1969), 415-440. MR 40 \#644.

148. - Some questions of uniform distribution, Ann. of Math. (2) 94 (1971), 125-138. MR $44 \# 4187$.

149. Strict ergodicity in zero dimensional dynamical systems and the Kronecker-Weyl theorem mod 2, Trans. Amer. Math. Soc. 140 (1969), 1-33. MR 39 \# 1410.

150. The equicontinuous structure relation for minimal Abelian transformation groups, Amer. J. Math. 90 (1968), 723-732. MR 38 \#702.

151. W Well distributed sequences of integers, Trans. Amer. Math. Soc. 161 (1971), 63-70.

MR $44 \# 2715$.

152. J. Ville, Etude critique de la notion de collectif, Gauthier-Villars, Paris, 1939.

153. B. Volkmann, Über Hausdorffsche Dimensionen von Mengen, die durch Zifferneigenschaften charakterisiert sind. VI, Math. Z. 68 (1958), 439-449. MR 20 \#7008. 
154. P. Walters, Invariant measures and equilibrium states for some mappings which expand distances, Univ. of Warwick, 1976, 52 pp. (preprint).

155. - Lectures on ergodic theory, Lecture Notes in Math., vol. 458, Springer-Verlag, Berlin and New York, 1975.

156. B. Weiss, Normal sequences as collectives, Proc. Conf. on Ergodic Theory and Topological Dynamics (Univ. of Kentucky, 1971), Math. Dept., Univ. of Kentucky, Lexington, Ky., pp. 79-80.

157. - Topological transitivity and ergodic measures, Math. Systems Theory 5 (1971), 71-75. MR 45 \#5987.

158. H. Weyl, Über die Gleichverteilung von Zahlen mod Eins, Math. Ann. 77 (1916), 313-352.

159. A. N. Zemlyakov and A. B. Katok, Topological transitivity of billiards in polygons, Mat. Zametki 18 (1975), 291-300. (Russian)

160. E. Szemerédi, On sets of integers containing no $k$ elements in arithmetic progression, Acta Arith. 27 (1975), 199-245. MR 51 \#547.

161. H. Furstenberg, Ergodic behaviour of diagonal measures and a theorem of Szemerédi on arithmetic progressions. I-III, Hebrew Univ., Jerusalem (preprint).

162. K. Roth, Sur quelques ensembles d'entiers, C. R. Acad. Sci. Paris 234 (1952), 388-390. MR 13, 724 .

163. A. Y. Khinchin, Three pearls of number theory, Graylock Press, Rochester, New York, 1952. MR 13, 724.

164. R. L. Graham and B. L. Rothschild, $A$ short proof of van der Waerden's theorem on arithmetic progressions, Proc. Amer. Math. Soc. 42 (1974), 385-386. MR 48 \# 8257.

Department of Mathematics, Rice University, Houston, Texas 77001 